Journal of Patient-Centered

\title{
Abstracts From the 26th Annual Health Care Systems Research Network Conference, April 8-10, 2020
}

Follow this and additional works at: https://aah.org/jpcrr

Part of the Analytical, Diagnostic and Therapeutic Techniques and Equipment Commons, Diseases Commons, Health and Medical Administration Commons, Health Information Technology Commons, Medical Specialties Commons, Mental and Social Health Commons, Nursing Commons, and the Public Health Commons

\section{Recommended Citation}

Abstracts from the 26th annual Health Care Systems Research Network Conference, April 8-10, 2020. J Patient Cent Res Rev. 2020;7:64-137. doi: 10.17294/2330-0698.1762

Published quarterly by Midwest-based health system Advocate Aurora Health and indexed in PubMed Central, the Journal of Patient-Centered Research and Reviews (JPCRR) is an open access, peer-reviewed medical journal focused on disseminating scholarly works devoted to improving patient-centered care practices, health outcomes, and the patient experience. 


\section{Abstracts From the 26th Annual Health Care Systems Research Network Conference, April 8-10, 2020}

The Health Care Systems Research Network (HCSRN) is made up of communitybased care delivery systems with a shared mission to improve health and health care through research. The network's annual conference serves as a forum for attendees to disseminate study findings, stimulate collaborations, and share insights about conducting research in real-world care settings. Although this year's live conference was cancelled to help slow the spread of COVID-19, the oral and poster abstracts accepted for presentation at HCSRN 2020 are published in this open access supplement of Journal of Patient-Centered Research and Reviews, the official scientific journal of the conference. J Patient Cent Res Rev. 2020;7(1):64-137.

\section{LATE-BREAKING RESEARCH}

OA5.03

The NIA IMPACT Collaboratory: Transforming Dementia Care Through Embedded Pragmatic Clinical Trials

Leah Hanson, ${ }^{1}$ Leah Tuzzio, ${ }^{2}$ James Fraser, ${ }^{2}$ Eric Larson $^{2}$

${ }^{1}$ HealthPartners, Bloomington, $\mathrm{MN} ;{ }^{2}$ Kaiser Permanente Washington, Seattle, WA

Background: More than 5 million Americans are living with Alzheimer's disease or a related dementia, and that number is expected to triple in the next three decades. People with dementia (PWD) have higher rates of hospital stays, emergency room visits, skilled nursing facility stays, and overall health care utilization. Caregivers of PWD experience increased anxiety, depression, and stress. It is imperative that health systems address this large and growing unmet need to improve symptom management for PWD and minimize caregiver strain. A disconnect between research evidence showing that nonpharmacologic interventions improve outcomes for PWD and their caregivers and real-world health care systems has stymied the adoption of promising interventions. Embedded pragmatic clinical trials (ePCTs) have the potential to accelerate the translation of evidencebased interventions into clinical practice.

Methods: The mission of the National Institute on Aging (NIA) IMPACT Collaboratory is to build the nation's capacity to conduct pragmatic clinical trials of nonpharmacologic interventions embedded within health care systems for PWD and their caregivers. Through 6 administrative and management teams and 10 working group cores, IMPACT aims to develop and disseminate best practice research methods, support the design and conduct of pragmatic trials (including funding of pilot studies), build investigator capacity through training and knowledge generation, catalyze collaboration among stakeholders including health care providers and investigators, and ensure culturally tailored interventions and engagement of people from diverse and underrepresented backgrounds.

Results: Leveraging the success of the collaboratory, IMPACT will develop the necessary support and resources specific to the unique needs of PWD and their caregivers. People living with dementia receive care across a spectrum of settings, including not just medical systems but their places of residence and programs to support remaining at home. Each of these settings has its own challenges to implementing ePCTs in this population.

Conclusion: The NIA IMPACT health care systems core is building a national resource devoted to the intersections between ePCTs for persons with dementia and the settings in which they receive health care. Our group seeks to engage investigators and sites within the HCSRN and other health systems to establish a collaborative to support and facilitate the conduct of ePCTs among PWD and their caregivers.

OA5.04

Testing of a Prototype Clinical Decision Support Tool to Combat Clinical Inertia at the Point of Care: Identifying and Closing Health and Care Gaps for Patients With Cardiometabolic Conditions

Hannah Husby, ${ }^{1}$ Jake Delatorre-Reimer, ${ }^{1}$ Farah Refai, ${ }^{1}$ Xiaowei (Sherry) Yan, ${ }^{1}$ Karen MacDonald, ${ }^{2}$ J.B. Jones, ${ }^{1}$ Madina Gbotoe, ${ }^{1}$ Kevin Knobel ${ }^{1}$

${ }^{1}$ Sutter Health, Walnut Creek, CA; ${ }^{2}$ AstraZeneca, San Francisco, CA 
Background: Clinical inertia, defined as the failure to modify therapy when clinically indicated, is a key contributing factor for why patients do not meet guideline-recommended goals and for poorly controlled health outcomes. In a previous investigation, we developed an algorithm to identify patients who potentially require new or escalated treatment using electronic health record (EHR) data for patients with cardiometabolic conditions (ie, diabetes, hypertension, and dyslipidemia). We refined the algorithm for point-of-care delivery with clinician feedback. Herein, we describe our experience field-testing the prototype clinical decision support algorithm and its presentation in a real-world clinical setting. Methods: The algorithm, derived based on EHR structured fields (ie, disease diagnosis, lab values, and medication order and adherence), identifies a patient's unmet goals and the need for gap-related action based on biometric values and treatment guidelines (ie, health gap), and compares these to medication orders and adherence (ie, care gap). Research staff completed chart reviews of patients the day before their scheduled office visit with pilot clinicians. A paper report was generated based on the assessment and given to the clinician prior to them entering the exam room with the patient. Clinicians made decisions on how to best act on the presented data and proceeded to share feedback with the research team on how to improve the algorithm and presentation.

Results: To date, 81 patients of 2 primary care clinicians were assessed. Of the 81 patients, $48(59 \%)$ were identified as having a health and/or care gap, and clinicians agreed with 36 $(75 \%)$ of these assessments. Pilot clinicians reported that the algorithm output helped set an agenda for the encounter and led to more direct data-driven conversations with patients about their condition(s). Reasons why alerts were not applicable/ not acted on included individual patient characteristics and history, age, and higher-priority topics.

Conclusion: Initial testing of this algorithm shows promise that clinicians are receptive to using clinical decision support tools that help deliver guideline-based care. Additional testing is needed to further refine the algorithm and presentation, and to determine its potential impact on clinician workflow efficiency and patient health outcomes.

\section{P1.07 \\ Development, Feasibility, and Preliminary Validation of a Spanish-Language Version of the TAPS Tool}

\section{Katherine Sanchez ${ }^{1}$}

\section{${ }^{1}$ Baylor Scott \& White Health, Dallas, TX}

Background: The TAPS Tool is a validated 2-stage screening and brief assessment in primary care for unhealthy substance use. We report on the development of a Spanish-language version of the TAPS Tool and its preliminary evaluation in a Spanish-speaking, NIH-designated health disparity population seen in community health centers. This project involved the adaptation of the TAPS Tool into Spanish, its development on a self-administered mobile/tablet technology platform, and an empirical pilot study of its feasibility, acceptability, and preliminary validity in a Spanish-speaking primary care sample.

Methods: Participants were adult primary care patients, age 18 years or older, with Spanish as their primary language $(\mathrm{N}=10$ for development/refinement using qualitative cognitive interviewing; $\mathrm{N}=100$ for the preliminary validation study). The Spanish-language TAPS Tool was administered in both interviewer- and self-administered format (in random order). We examined disclosure of substance use on TAPS by administration format and compared it with established measures (modified Composite International Diagnostic Interview [CIDI]; World Health Organization's Alcohol, Smoking and Substance Involvement Screening Test [ASSIST]) for identifying substance use and substance use disorders.

Results: The Spanish-language TAPS was feasible to use, and participants reported high levels of acceptability. The sample had low rates of past 12 -month substance use $(11 \%$ tobacco, $28 \%$ risky alcohol, $4 \%$ illicit drugs, $1 \%$ nonmedical prescription drugs) and use disorders (7\% tobacco, $2 \%$ alcohol, $1 \%$ other substances). The self-administered TAPS elicited 1,3 , and 1 additional disclosures of tobacco, risky alcohol, and marijuana use, respectively, as compared to the interviewer-administered TAPS. Rates of disclosure on TAPS were similar to those on established measures for past 12-month and 3-month time frames.

Conclusion: Deployment of linguistically accurate and culturally relevant screening and assessment in primary care could improve detection of substance use problems in Spanishspeaking patients and open new opportunities for delivering primary care-based behavioral interventions and referrals to specialty treatment when clinically indicated. The current study represents a starting point for expanding the availability of the TAPS Tool beyond its original English-language version into Spanish. The Spanish-language TAPS Tool could expand options for substance use screening in primary care settings with Spanish-dominant/preferred populations.

\section{P3.05}

Assessing Heart Disease in a Large Population of Adults With Down Syndrome, 1991-2019

\author{
Veronica Fitzpatrick, ${ }^{1}$ Anne Rivelli, ${ }^{1}$ Brian Chicoine ${ }^{2}$
}

\section{${ }^{1}$ Advocate Health Care, Park Ridge, IL; ${ }^{2}$ Advocate Medical Group, Park Ridge, IL}

Background: Down syndrome (DS) is characterized by intellectual disabilities, dysmorphic features, and comorbid conditions that result from increased genetic material of the $21^{\text {st }}$ chromosome. As recently as 1983 , an adult with DS was only expected to live until age 25 , but with advances in medicine, many individuals with DS are currently living up to 60 years. This poses new challenges for the individual, 
families, and clinical care teams, particularly the prevention and treatment of aging-related conditions. Research into the clinical trajectory of adults with DS is warranted to improve the health of adults with DS and update current health care guidelines. The purpose of this study was to 1) describe demographic factors and calculate prevalence of clinical conditions related to heart disease among the adult DS sample population, and 2) compare demographic and clinical heart disease conditions between the DS sample population and the general population.

Methods: Utilizing a retrospective, descriptive-cohort study design, analyses were based on 28 years of data collected on the patient population of Advocate Health Care's Adult Down Syndrome Center. Prevalence of disease and risk conditions are reported as counts (and percentages) with corresponding odds ratio (OR) indicating odds of diagnosis among the DS sample compared to the general population. Corresponding Pearson chi-squared P-values were calculated to represent statistically significant differences between prevalence of diagnoses in the DS sample compared to the general population. In cases where prevalence was low $(<5)$, Fisher's exact test P-values were calculated.

Results: Overall, adults with DS had significantly lower odds of coronary heart disease (OR: 0.432, $\mathrm{P}<0.0001$ ), angina (OR: 0.047. $\mathrm{P}<0.0001$ ), and myocardial infarction (OR: 0.012, $\mathrm{P}<0.0001$ ), with lower odds of the heart disease risk factors diabetes mellitus (OR: 0.535, $\mathrm{P}<0.0001)$ and hypertension (OR: 0.071, $\mathrm{P}<0.0001$ ). Adults in the DS sample have greater odds of heart failure (OR: 1.48, $\mathrm{P}<0.0001$ ), stroke (OR: 1.259, $\mathrm{P}<0.166$ ), being overweight (OR: 1.219, $\mathrm{P}<0.0002$ ) and being obese (OR: 1.324, $\mathrm{P}<0.0001)$ as compared to the general population.

Conclusion: Targeted clinical care pathways focus on reducing risk factors for heart disease conditions and events; however, our prevalence findings suggest that typical risk factors and heart-related conditions look very different in a DS population. Therefore, clinical care guidelines should more accurately reflect the conditions most prevalent in the DS population.

\section{P3.06}

\section{Control of Risk Factors for the Secondary Prevention of Cardiovascular Disease}

\author{
Nicole Glowacki, ${ }^{1}$ Rasha Khatib ${ }^{1}$
}

\section{${ }^{1}$ Advocate Research Institute, Advocate Health Care, Oak Lawn, IL}

Background: Cardiovascular disease is a leading cause of death and disability worldwide and is particularly prevalent among patients with hypertension. Clinical practice guidelines recommend controlling multiple risk factors to reduce cardiovascular events in these patients. Lifestyle interventions and pharmacotherapy aimed at controlling risk factors show effectiveness in randomized controlled trials. The level of risk factor control in clinical real-world practice is not well documented, especially in primary care where most of these patients are managed.

Methods: To describe real-world control of cardiovascular risk factors in patients diagnosed with hypertension and free cardiovascular disease in primary care, we retrospectively analyzed electronic medical record data from a large health care system in Illinois. Data were collected on patients with at least 2 primary care encounters in 2018 and who were diagnosed with hypertension for at least 6 months based on ICD-10 codes. Patients diagnosed with coronary artery disease, stroke, heart failure, or chronic kidney disease were excluded. Clinical practice guidelines recommend a blood pressure goal of $<150 / 90 \mathrm{mmHg}$ in patients 60 years of age or older and $<140 / 90 \mathrm{mmHg}$ in diabetic patients or those younger than age 60 , a low-density lipoprotein cholesterol (LDL-C) goal of $<100 \mathrm{mg} / \mathrm{dl}$, glycemic control (defined as hemoglobin A1c of $<7 \%$ ), and not smoking. The last available measurement during the study period was used to assess control of each cardiovascular risk factor.

Results: A total of 4595 patients managed in primary care and diagnosed with hypertension were included in the analysis. Mean age was $61 \pm 13.7$ years, $65.9 \%$ were females, 49.9\% were Caucasian, and $95.1 \%$ reported English as a first language. Blood pressure goals were achieved in $73.4 \%$ of the population. LDL-C goals were achieved in $47.9 \%$ of patients. Glycemic control was achieved in $94.2 \%$ of patients, and $89.6 \%$ were not smokers. Overall, $31.4 \%$ of patients with hypertension achieved control across the 4 risk factors.

Conclusion: A significant proportion of patients diagnosed with hypertension and managed in primary care failed to achieve targets for 4 major modifiable cardiovascular risk factors in clinical practice. New approaches to achieve optimal secondary prevention treatment goals are required to translate evidence into practice.

P5.10

Ejection Fraction Extraction From Echocardiography Reports With an Application of Computerized Algorithm

Xiangyi Xu, ${ }^{1}$ Wendi Knapp, ${ }^{1}$ Souvik Das, ${ }^{1}$ Anthony Sturzu, ${ }^{1}$ Kimberly Buss, ${ }^{1}$ Kelly Miller, ${ }^{1}$ Erica Weirich, ${ }^{1}$ Kaartiga Sivanesan, ${ }^{1}$ John Smucny, ${ }^{1}$ Margaret MacMillan, ${ }^{1}$ Xiaowei (Sherry) Yan ${ }^{1}$

\section{${ }^{1}$ Sutter Health, Walnut Creek, CA}

Background: Left ventricular ejection fraction (LVEF) is an important diagnostic indicator for the classification of heart failure and is used in determining clinical treatment. However, LVEF is documented in free-text reports with considerable variations among how and where it is reported, which hinders the utilization of LVEF. We developed a regular-expressionbased algorithm to automatically extract ejection fraction (EF) values from echocardiographic reports and conducted comprehensive validation. 
Methods: We conducted a 2-phase study in the algorithm development and validation. The outcomes of the study included EF value and EF quality, which refers to domains from where EF was extracted. At the first phase, a regularexpression-based algorithm was developed by comparing outcomes derived from the algorithm with those manually reviewed by an experienced physician on 112 randomly selected reports. The refined algorithm was further validated in the second phase. At the second phase, 512 reports were randomly selected from all available data spanning January 1, 2017-December 31, 2018, stratified by clinical departments, and manually reviewed by 7 physicians. Each report was reviewed by 2 physicians. We evaluated the interreviewer agreement on both outcomes and also compared the algorithm-derived values with the "gold standard," defined as the agreed-on EF value between 2 reviewers.

Results: Our algorithm was initially developed using a list of keywords, including left ventricular ejection fraction, LVEF, $\mathrm{EF}, \mathrm{LV}$ systolic function, and variants of those expressions, created based on mining from 13,681 echocardiographic reports. At the first phase, both the physician and the algorithm identified EF values from 88 of 112 reports (78.6\%), showing $100 \%$ consistency of identification fidelity. Among those 88 reports, the accuracy of EF value extracted by the algorithm was $97.5 \%$. In phase 2, the interreviewer consistency was $93.9 \%$ for $\mathrm{EF}$ value and $82.5 \%$ for EF quality. Compared to gold standard on 499 reports, the algorithm accuracy of EF value was $97.2 \%$, consistent with phase 1 validation.

Conclusion: Cardiovascular domain knowledge is the key in developing highly accurate natural language process algorithms for extracting EF from free texts. The 2-phase validation has ensured the accuracy and validity of the algorithm before implementing it throughout the health care system.

\section{P6.03}

\section{Prescription Opioid Distribution Trends After the Legalization of Recreational Marijuana in Colorado, 2007-2017}

\section{Amalie Kropp Lopez, ${ }^{1}$ Stephanie Nichols, ${ }^{2}$ Daniel Chung ${ }^{1}$ Kenneth McCall, ${ }^{2}$ Brian Piper ${ }^{1}$}

${ }^{1}$ Geisinger Commonwealth School of Medicine, Scranton, PA; ${ }^{2}$ University of New England, Portland, ME

Background: Opioid-related overdoses and overprescribing continue to be an issue within the United States. Further consideration of nonopioid alternatives as a substitute to treat chronic noncancer pain and in the treatment of opioid use disorders (OUD) is warranted. We aimed to examine the association between the legalization of Colorado's recreational marijuana and prescription opioid distribution trends. Two states that have not legalized recreational cannabis were selected for comparison.

Methods: The U.S. Drug Enforcement Administration's Automation of Report and Consolidated Orders System
(ARCOS) was used to examine 9 pain medications oxycodone, fentanyl, morphine, hydrocodone, hydromorphone, oxymorphone, tapentadol, codeine, and meperidine - and 2 OUD medications - methadone and buprenorphine - from 2007 to 2017 in Colorado, Utah, and Maryland. Drug weights were extracted, examined, and graphed. Medications were converted to their oral morphine milligram equivalents (MME) using standard conversion factors.

Results: Colorado reached a peak of pain MME weight in 2012 and had a $-11.66 \%$ reduction from 2007 to 2017. Utah had a $+9.64 \%$ increase in pain medication distribution, and Maryland had a $-6.02 \%$ reduction. As for medications used for OUD, Colorado, Utah, and Maryland saw a $+19.42 \%$ increase, a $-31.45 \%$ reduction, and a $+66.56 \%$ increase, respectively, from 2007 to 2017. Analysis of the interval pre- (2007-2009) versus post-marijuana legalization (20132017) was completed. Statistically significant changes for pain medications were observed for Colorado $(\mathrm{P}=0.033)$ and Maryland $(\mathrm{P}=0.007)$ but not Utah $(\mathrm{P}=0.659)$. Analysis of the OUD medications identified significant changes for Colorado ( $\mathrm{P}=0.0003)$ and Maryland $(\mathrm{P}=0.0001)$ but not Utah $(\mathrm{P}=0.0935)$. Over the decade, Colorado's opioid distribution was predominantly $(72.49 \%)$ for pain with one-quarter (27.51\%) for an OUD. Utah distributed $61.00 \%$ for pain and $39.00 \%$ for OUD. However, Maryland was one-third (37.89\%) for pain and over three-fifths $(62.11 \%)$ for an OUD. Conclusion: This study found that there has been a significant decrease in prescription opioid distribution after the legalization of marijuana in Colorado. Colorado had a larger decrease in opioid distribution after 2012 than Utah or Maryland. Therefore, marijuana should be considered as an alternative treatment for chronic pain and reducing use of opioids. Also, when combined with other research reviewed in this study, marijuana use may reduce the overdose death rate. Additional research with more comparison states is ongoing.

\section{P6.04 \\ Impact of Sustained Weight Loss on Incident Type 2 Diabetes}

Craig Wood, ${ }^{1}$ Lisa Bailey-Davis, ${ }^{1}$ Abhilasha Ramasamy, ${ }^{2}$ Neeraj lyer, ${ }^{2}$ Gabriel Smolarz, ${ }^{2}$ Jacob Mowery, ${ }^{1}$ Adam Cook, ${ }^{1}$ Peter Benotti, ${ }^{1}$ Christopher Still ${ }^{1}$

${ }^{1}$ Geisinger Health, Danville, PA; ${ }^{2}$ Novo Nordisk USA, Plainsboro, NJ

Background: The risk of developing type 2 diabetes (T2D) increases in direct proportion with higher body weight and decreases with body weight loss. However, the success of achieving clinically meaningful weight loss is highly variable, and the impact of sustained weight loss versus weight stability or weight regain on future T2D risk is unclear.

Methods: Adult primary care patients with body mass index (BMI) of $>27 \mathrm{~kg} / \mathrm{m}^{2}$ were retrospectively evaluated over a 2-year referent period occurring between 2002 and 2018. 
Those with baseline weight, 1-year weight, and 2-year weight measured during the referent period were categorized into overweight/obesity maintainers (OM, maintained weight within $3 \%$ of baseline), weight loss maintainers (WLM, lost $\geq 5 \%$ weight in first year and maintained $\geq 80 \%$ of weight loss by the end of second year), and weight loss rebounders (WLR, lost $\geq 5 \%$ weight in first year and regained $>20 \%$ of weight loss by the end of second year). The postreferent period (up to 10 years, ending in 2018) was reviewed to identify incident T2D. Cox regression adjusted for age, sex, and baseline BMI was used to compare the time until incident T2D between the weight change group and by the overall magnitude of maintained weight loss within the WLM group. Results: The 49,327 qualifying patients comprised 57\% females, a mean age of 49.8 years (SD: 15.2), a mean BMI of 34.9 (SD: 5.2), and a median follow-up of 6.4 years. Weight change groups included $67 \%$ OM $(\mathrm{n}=33,260), 20 \%$ WLR $(\mathrm{n}=9630)$, and $13 \%$ WLM $(\mathrm{n}=6437)$. Overall incidence of T2D for the overall population was $6.3 \%$ at 3 years, $10.1 \%$ at 5 years, and $18.9 \%$ at 10 years. As compared to the OM group, incident T2D was lowest for the WLM group (adjusted hazard ratio [aHR]: 0.67, 95\% CI: $0.61-0.73, \mathrm{P}<0.0001)$ but was not reduced in the WLR group (aHR: $0.95,95 \%$ CI: 0.89 $1.02, \mathrm{P}=0.163$ ). Within the WLM group, the magnitude of maintained weight loss was associated with a lower incident T2D (aHR: 0.72 for each additional 5\% of maintained weight loss, 95\% CI: 0.64-0.81, $\mathrm{P}<0.0001$ ).

Conclusion: Weight loss is most effective at reducing risk of future T2D when weight loss is maintained and weight regain is minimized. T2D risk reduction was directly related to the magnitude of sustained weight loss.

\section{P14.06}

\section{AHRQ EPC Program Initiatives to Improve Utility of Evidence Reviews for Health Systems}

\author{
Celia Fiordalisi, ${ }^{1}$ Kelly Vander Ley, ${ }^{1}$ Amanda Borsky, ${ }^{2}$ \\ Cathy Gordon, ${ }^{1}$ Stephanie Chang ${ }^{2}$
}

${ }^{1}$ Scientific Resource Center, Agency of Healthcare Research and Quality, Portland, OR; '2Evidencebased Practice Center Program, Agency of Healthcare Research and Quality, Rockville, MD

Background: The Agency for Healthcare Research and Quality (AHRQ) Evidence-based Practice Center (EPC) program has been producing comprehensive systematic evidence reviews of health care literature for over two decades, traditionally serving needs of clinical practice guideline developers and professional societies. Recently, AHRQ has worked to speed the adoption of evidence into the practices of learning health systems. The ongoing evolution of EPC products to address questions of usability, implementation, applicability, relevance, and timeliness is a major component of this effort.

Methods: The AHRQ EPC program has engaged health systems through a variety of methods. Information on the improvement of evidence reviews has been gathered through the AHRQ-convened ACTION Network Learning Health Systems panel, qualitative interviews with health systems operational leadership, and sessions at clinical conferences.

Results: Engagement with health system leaders has resulted in several EPC program efforts to adapt the existing framework for presenting systematic reviews and explore alternative evidence products that can be completed on a shorter timeline. The identified need to make information accessible has led to a revised evidence summary emphasizing actionable findings and visual representations of data. Customizable interactive data visualization techniques also are being developed to provide health systems with the ability to start with higherlevel summary statements with optional deeper data dives. Health systems underscored the importance of including contextual factors for implementation and applicability in the evidence synthesis. To address this need, the program is actively discussing the reformulation of the traditional hierarchy of evidence to place more value on evidence from pragmatic trials and observational studies that provide information on factors like implementation considerations, studied populations, and settings.

Conclusion: Each subsequent initiative has expanded and built upon the last. Collectively, they are reframing the presentation and methodologic approach of EPC reports to address the evidence needs of learning health systems. Current efforts range from expanding the breadth of considered evidence to developing data visualization solutions for making evidence more accessible for its intended use. We encourage collaboration with health systems of all sizes and hope HCSRN members will nominate review topics and provide input to make AHRQ EPC products most useful to their systems.

\section{CARDIOVASCULAR DISEASE}

\section{OA1.01 \\ Prevalence of Apparent Treatment-Resistant Hypertension in a U.S. Integrated Health Care System}

Jaejin An, ${ }^{1}$ John Sim, ${ }^{1}$ Ran Liu, ${ }^{1}$ Rong Wei, ${ }^{1}$ Hui Zhou, ${ }^{1}$ Kristi Reynolds ${ }^{1}$

\section{${ }^{1}$ Kaiser Permanente Southern California, Pasadena, CA}

Background: The 2017 American College of Cardiology/ American Heart Association (ACC/AHA) blood pressure (BP) guideline lowered BP treatment goals from $<140 / 90$ $\mathrm{mmHg}$ to $<130 / 80 \mathrm{mmHg}$ for most adults with hypertension. We compared prevalence of apparent treatment-resistant hypertension (aTRH) according to the seventh report of the Joint National Committee (JNC 7) or the 2017 ACC/AHA guideline in an integrated health care delivery system. 
Methods: We identified individuals $\geq 18$ years old with hypertension from the Kaiser Permanente Southern California hypertension registry between July 1, 2014, and June 30, 2015. aTRH was defined as either BP above goal while taking 3 or more classes of antihypertensive medication or taking 4 or more classes of antihypertensive medication regardless of BP level. We used the lowest BP measurement from the first outpatient visit encounter after exposure to 3 or more classes of antihypertensive medication concurrently. A secondary analysis was conducted requiring use of a thiazide diuretic for the definition of aTRH. Patient clinical characteristics and antihypertensive medication use were described using electronic health records.

Results: We included 469,509 patients with treated hypertension (mean age: 65 years [SD: 12 years]; race: $46 \%$ white, $26 \%$ Hispanic, $13 \%$ black, and $12 \%$ Asian). Prevalence of aTRH was $16.9 \%$ and $21.8 \%$ according to the JNC 7 and 2017 ACC/AHA guidelines, respectively $(\Delta: 4.9 \%$ [95\% CI: $4.7 \%-5.0 \%]$ ). The newly classified patients with aTRH according to the 2017 ACC/AHA guideline were younger and had fewer comorbidities compared with patients with aTRH per JNC 7. By requiring a thiazide diuretic to be considered as aTRH, the prevalence of aTRH decreased to $10.4 \%$ and $13.8 \%$ according to JNC 7 and 2017 ACC/AHA guidelines, respectively. The most frequent 3-drug antihypertensive combination was an angiotensin-converting enzyme inhibitor (or angiotensin receptor blocker), beta blocker, and thiazide diuretic. Among patients with aTRH, 1.9\% received a longacting thiazide-like diuretic (chlorthalidone or indapamide), and 5.6\% received a mineralocorticoid receptor blocker (spironolactone or eplerenone).

Conclusion: As expected, the prevalence of aTRH increased using the more stringent BP goals of the 2017 ACC/AHA guideline. The use of recommended long-acting thiazide-like diuretics and mineralocorticoid receptor blockers for aTRH was suboptimal. Future studies may be necessary promoting the use of effective pharmacotherapy in aTRH.

OA1.02

Providers' Prescribing Behavior for Lipid-Lowering Therapy After Receiving Patients' Positive Genetic Test for Familial Hypercholesterolemia

Jing Hao, ${ }^{1}$ Nan Chen, ${ }^{1}$ Dina Hassen, ${ }^{1}$ Tracey Klinger, ${ }^{1}$ Megan McMinn, ${ }^{1}$ Dustin N. Hartzel, ${ }^{1}$ David Veenstra, ${ }^{2}$ Scott Spencer, ${ }^{2}$ Marc S. Williams, ${ }^{1}$ Susan R. Snyder, ${ }^{3}$ Josh F. Peterson, ${ }^{4}$ Victoria Schlieder, ${ }^{1}$ Laney K. Jones ${ }^{1}$

${ }^{1}$ Geisinger Health, Danville, PA; ${ }^{2}$ University of Washington, Seattle, WA; ${ }^{3}$ Georgia State University, Atlanta, GA; ${ }^{4}$ Vanderbilt University, Nashville, TN

Background: Familial hypercholesterolemia is an inherited condition causing significant lifelong elevation in low-density lipoprotein cholesterol (LDL-C), which results in increased risk of premature cardiovascular disease. The MyCode initiative is a precision medicine program at Geisinger that returns medically actionable results from exome sequencing. Our objective was to evaluate providers' prescribing behavior for lipid-lowering therapies (LLT) after receiving patients' positive genetic result for familial hypercholesterolemia.

Methods: We conducted a retrospective study on MyCode participants who received familial hypercholesterolemia results. The observation period was from 2 years prior to the patient receiving a positive genetic result for familial hypercholesterolemia to January 16, 2019. Patients' health records, including LLT prescriptions and LDL-C values, were obtained from the electronic health record supplemented by chart review. Descriptive analysis was conducted to demonstrate changes in patients' LLT management and LDL-C level before and after genetic result disclosure.

Results: In preliminary analysis, we analyzed 98 MyCode patients who received positive familial hypercholesterolemia results; 76 were already on LLT and 22 were not on any LLT before disclosure. After receiving genetic results of familial hypercholesterolemia, 30 patients received more intensive treatment, 34 patients had no change in their LLT, and 34 patients were not on LLT at the end of our observation period (15 discontinued LLT and 19 continued not to receive LLT). We found that 8 patients were at LDL-C goal (of either $<100$ $\mathrm{mg} / \mathrm{dL}$ for primary prevention or $<70 \mathrm{mg} / \mathrm{dL}$ for secondary prevention) before disclosure; 3 of them were no longer at goal after disclosure. Among the 50 patients who were not at goal before disclosure, 34 experienced improvements in their LDL-C level (11 patients met goal), 9 patients' LDL-C level increased, and 7 patients had no change after disclosure. The remaining 40 patients did not have sufficient LDL-C data for classification.

Conclusion: Among patients receiving genetic diagnosis of familial hypercholesterolemia, one-third had increased intensity in LLT and about 35\% experienced improvements in their LDL-C level. However, after receiving genetic results of familial hypercholesterolemia, an increased number of patients were not on LLT and most patients were still not on goal. Further study is needed to understand the factors impacting treatment.

\section{OA1.03 \\ Randomized Controlled Trial Comparing Self- Guided Versus Dietitian-Led Approach Using Web-Based Tools to Lower Blood Pressure: Study Design and Rationale}

Alex Chang, ${ }^{1}$ Heather Bonaparte, ${ }^{1}$ Christina Yule, ${ }^{1}$ Allison Naylor, ${ }^{1}$ Sara Kwiecien, ${ }^{1}$ Mohamed Taher, ${ }^{1}$ Andrea Nale, ${ }^{1}$ Stephen Juraschek, ${ }^{1}$ Charlotte Collins, ${ }^{1}$ Lisa Bailey-Davis ${ }^{1}$

\section{${ }^{1}$ Geisinger Health, Danville, PA}

Background: Weight loss, consumption of a Dietary Approaches to Stop Hypertension (DASH) dietary pattern, 
reduced sodium intake, and increased physical activity have been shown to lower systolic blood pressure (SBP). Use of website- or smartphone-based tools could be potentially scalable solutions to improve lifestyle behaviors and lower SBP, but little data exist to support their use in clinical practice. Methods: In an ongoing 2-arm randomized controlled trial in the Geisinger system, we compare the efficacy of a dietitianguided approach to a self-guided approach in lowering 24hour ambulatory blood pressure (ABP) in overweight/obese patients with 24-hour SBP of 120-160 mmHg and on 0-1 blood pressure medication. As part of standard of care, participants with outpatient SBP of $\geq 130 \mathrm{mmHg}$ are identified using Geisinger electronic health record and health plan wellness data, then invited to complete 24-hour ABP. Qualifying participants are invited to complete a web-based questionnaire (Viocare $^{\circledR}$ ) that provides personalized recommendations to improve dietary quality, access to a comprehensive weight management program (www.bmiq.com), and a smartphone app (Lose It!). Participants are then randomized 1:1 to a selfguided arm or a dietitian-led arm, which additionally receives weekly telephone calls from registered dietitian nutritionists trained in motivational interviewing. Primary outcome is 3-month change in 24-hour SBP.

Results: Out of 210 patients who have thus far completed ABP, 140 were eligible for the trial, 85 consented for the trial, and 81 were randomized after completing the run-in period. In a preplanned interim analysis of the first 34 randomized patients, $28(82 \%)$ completed the 3-month study visit. Mean (SD) changes from baseline to 3 months were $-8.0 \mathrm{mmHg}$ (9.9) for 24-hour SBP, -5.4 kg (4.5) for weight, -6.1 cm (7.4) for waist circumference, and 11.2 units (12.0) for Healthy Eating Index score.

Conclusion: Confirmation of elevated blood pressure using ABP may provide an opportunity to engage patients in making lifestyle modifications, as $58 \%$ of eligible patients after $24-$ hour ABP screening have been successfully randomized with strong preliminary results. Completion of the study is expected by the end of 2020 and will determine the efficacy of a self-directed vs dietitian-led approach to lower 24-hour SBP using web-based tools in an integrated health system.

\section{OA1.04}

\section{Utilizing Core Electronic Health Record Functionalities to Prevent Cardiovascular Disease Among Underserved Patients}

\section{Christopher Rogers ${ }^{1}$}

\section{${ }^{1}$ Seton Hall University, South Orange, NJ}

Background: Preventable cardiovascular disease (CVD) disproportionately affects low-income Medicaid-eligible populations. Electronic health record (EHR) use has been proved to increase the likelihood of receiving recommended primary preventive care for CVD. Several studies have shown that EHRs integrated with clinical decision support functions help physicians to use alerts, reminders, and shared health information to proactively prevent chronic conditions in high-risk safety-net patients. Despite these important findings highlighting the role of EHRs in preventing chronic diseases in high-risk patients, the literature remains mixed on whether EHRs can be instrumental in improving CVD outcomes.

Methods: Utilizing a nationally representative survey of ambulatory care visits by patients at risk for CVD in 2014 and 2015, binary logistic regression models examined differences in the associations between core EHR functionalities for public and population health, care coordination, patient engagement, and quality improvement on the provision of recommended CVD-preventive health services provided at primary care visits for adults at risk for CVD who had Medicaid and those with private insurance.

Results: Primary care providers caring for at-risk patients with Medicaid were significantly less likely to adopt fully functional certified EHR technology compared to PCPs caring for private insurance patients (75\% vs $82 \%$, $\mathrm{P}<0.01)$. Overall Medicaid and private insurance patients, respectively, had low rates of diet/nutrition counseling, exercise counseling, hemoglobin A1c test, lipids/cholesterol screening, and weight reduction counseling. Medicaid patients were significantly less likely to receive recommended blood pressure screening compared to private insurance patients (95\% CI: 0.205-0.931). Among preventive visits for Medicaid patients at risk for CVD, significant associations were found between select recommended CVD-preventive services and public and population health management, care coordination, and patient engagement.

Conclusion: Study findings demonstrate concerns about the rates of recommended CVD-preventive services at primary care visits for Medicaid patients at risk for CVD, which imply gaps in their care. Increased physician adoption of fully functional certified EHR technology may facilitate recommended preventive CVD screening for low-income, underserved patients with Medicaid.

\section{P3.01}

Direct Oral Anticoagulant Use in Patients With Atrial Fibrillation at Extreme Body Weight: What Is the Scope of the Problem?

Stephen Voyce, ${ }^{1}$ Patrick Roman, ${ }^{1}$ Joseph Chronowski, ${ }^{1}$ Eric Wright, ${ }^{1}$ Michael Gionfriddo, ${ }^{1}$ Eric Kowalek, ${ }^{1}$ Danielle Kuhn, ${ }^{1}$ Amanda Boyer ${ }^{1}$

\section{${ }^{1}$ Geisinger Health, Danville, PA}

Background: Multiple medical society guidelines recommend direct oral anticoagulants (DOACs) over vitamin $\mathrm{K}$ antagonists (warfarin) for use in patients with atrial fibrillation. These agents are dosed according to renal function, but there is no alteration in dose for increased body weight or body mass index. Limited pharmacokinetic and pharmacodynamics data suggest that patients at the upper extremes of body 
weight (UEBW) $(>120 \mathrm{~kg}$ and/or body mass index of $>40 \mathrm{~kg} / \mathrm{m}^{2}$ ) have decreased drug exposures, reduced peak concentrations, and shorter half-lives, which raises concerns about underdosing the population that falls under UEBW. Previous trials have looked at clinical efficacy based on posthoc analyses, and no study has described the demographics or the prescribing patterns of DOACs in UEBW patients.

Methods: We utilized a retrospective review of the Geisinger Pharmacy and Epic electronic medical record database from 2010 to 2018 for patients with atrial fibrillation who were in the UEBW category. Diagnosis, medication profile, duration of therapy, efficacy, and safety results are to be analyzed.

Results: Of the 10,140 patients with atrial fibrillation prescribed DOACs, we identified 1763 (17.4\%) who fell under the UEBW category. Over the duration of the study, the number of patients treated with DOACs increased, but the percentage of UEBW patients prescribed DOACs remained stable. A higher percentage of UEBW men were treated with DOACs as compared to UEBW women (18.8\% vs $15.6 \%$ ).

Conclusion: $17.4 \%$ of individuals with atrial fibrillation treated with DOACs are UEBW. The number of UEBW patients treated with DOACs has increased over time. Men at UEBW are more likely to be treated with DOACs than women in the same category. As the study progresses, we will be looking to understand the clinical efficacy and safety of DOACs in UEBW patients.

\section{P3.02}

Assessing Anticoagulation Management and Shared Decision-Making Documentation From Providers Participating in the SUPPORT-AF Study

Laboni Hoque, ${ }^{1}$ Azraa Amroze, ${ }^{1}$ Vinit Gilvaz, ${ }^{1}$ Sonu Abraham, ${ }^{1}$ Amos Lal, ${ }^{2}$ Ajay Mishra, ${ }^{3}$ Sybil Crawford, ${ }^{1}$ Kathleen M. Mazor, ${ }^{1}$ David McManus, ${ }^{1}$ Alok Kapoor ${ }^{1}$

${ }^{1}$ Reliant Medical Group/University of Massachusetts Medical School/Fallon Health, Worcester, MA; ${ }^{2}$ Mayo Clinic, Rochester, MN; ${ }^{3}$ St. Vincent Hospital, Worcester, $M A$

Background: We previously tested an intervention featuring educational outreach with modified academic detailing (AD) to increase anticoagulation use in patients with atrial fibrillation. Currently, we compare providers receiving and not receiving $\mathrm{AD}$ in terms of inclusion of our $\mathrm{AD}$ educational topics and shared decision-making elements in documentation.

Methods: Physicians reviewed themes discussed with providers during $\mathrm{AD}$ and evaluated charts for evidence of shared decision-making. We then compared frequency of documentation of individual items for providers receiving AD compared with non-AD providers. To understand baseline documentation practices of $\mathrm{AD}$ providers, we also randomly selected encounters of $\mathrm{AD}$ providers before their AD participation.

Results: There were 113 eligible encounters in the 4 months after $\mathrm{AD}-36$ from $\mathrm{AD}$ providers and 77 from non-AD providers. We also identified 35 encounters from AD providers before participating in the intervention. Providers infrequently documented many reviewed items ( $\%$ documenting): anticoagulation mentioned (44\%); multiple options for anticoagulation (5\%); $\mathrm{CHA}_{2} \mathrm{DS}_{2}$-VASc score (11\%); and bleeding risk factors $(2 \%)$. Compared with nonAD providers, AD providers had statistically significant higher percentages for the following items: mention of anticoagulation (64\% vs 35\%); stroke risk (11\% vs $0 \%)$; anticoagulation benefits ( $8 \%$ vs $0 \%$ ); and patient involvement (70\% vs $0 \%$ ). There was no improvement, however, for $\mathrm{AD}$ providers compared with baseline documentation percentages. Conclusion: Providers infrequently documented important items in anticoagulation management and shared decisionmaking. AD participation did not improve documentation. Improving adoption of $\mathrm{AD}$ educational items may require more prolonged interaction with providers. Improving shared decision-making may require an intervention more focused on it and its documentation.

\section{P3.03}

\section{Nurse-Driven Multidisciplinary Rounds to Decrease 30-Day All-Cause Readmission Rate for Heart Failure}

J. Patrick Quiambao, ${ }^{1}$ Lillian Clowers, ${ }^{1}$ Dianna CurrierKirby, ${ }^{1}$ Maryam Jamshidi ${ }^{1}$

\section{${ }^{1}$ Kaiser Permanente Northwest, Portland, OR}

Background: Patients with heart failure are at a higher risk for 30-day all-cause hospital readmissions, and nurses play a critical role in reducing readmission rates.

Methods: During an 11-month period, the nurses of an acute care medical unit were provided with additional education and resources on heart failure, tools for educating patients with heart failure, and guidelines for multidisciplinary rounding. The team participating in rounding include the unit nurse manager, a nursing professional development educator, an acute care pharmacist or pharmacy intern, and the primary nurse. Physicians were included via text messages. Over the last 11 months, 30-day all-cause readmission rates were compared to rates prior to implementation of the multidisciplinary rounds.

Results: The spring 2019 readmission rate was $19.9 \%$, $5.1 \%$ below the target rate of $25 \%$. Nursing documentation of heart failure education provided to patients rose from less than $40 \%$ to more than $90 \%$.

Conclusion: Nurse-driven multidisciplinary rounds had a direct impact on the 30-day all-cause hospital readmission rate in spring 2019. Heart failure rounds resulted in patients receiving more consistent heart failure education and medication management per American Heart Association guidelines. Nursing-driven heart failure rounds resulted in improved patient heart failure education and increased nurse 
interaction with patients. These measures have contributed to the improved patient readmission rate. One can draw the conclusion that readmission rates will continue to improve as the program and processes become more hard-wired with nursing staff and other disciplines.

\section{P3.04 \\ Accuracy of Computer-Interpreted Electrocardiographic Diagnosis of Prior Anteroseptal Myocardial Infarction}

\section{Stephen Voyce, ${ }^{1}$ Humera Mohammmad, ${ }^{1}$ Gilhooley, ${ }^{1}$ Jason Lewis, ${ }^{1}$ Kathleen Cope ${ }^{1}$ \\ ${ }^{1}$ Geisinger Health, Danville, PA}

Background: Electrocardiograms (ECG) are commonplace in the treatment of patients. Of the millions of ECG performed annually, the majority are analyzed and interpreted by computer programs. Previous studies have analyzed some of the benefits and limitations of these computer programs. Special attention has not been paid to the diagnosis of previous anterior, septal, and anteroseptal infarction (ASMI). Although current computer algorithms use strict criteria in their interpretation, the accuracy of computer-interpreted ECG (CIE) diagnosis of prior ASMI and subsequent impact on the utilization of medical resources is unknown.

Methods: We performed a retrospective analysis of ECG performed from April 1, 2018, to April 30, 2019. Data was extracted from MUSE using a CIE keyword of ASMI. We excluded serial ECG. The CIE+ diagnoses were compared to physician-interpreted ECG (PIE). PIE and CIE were correlated with imaging (echocardiograms and/or nuclear) studies performed within 6 months of the ECG. A numerical analysis was conducted.

Results: A total of 2007 ECG were performed; 422 were ASMI; 125 were excluded. A total of 297 ECG (15.8\%) were CIE+ for prior ASMI; 168 of the 297 (56.6\%) had imaging studies. Physicians (PIE+) only agreed with $47.1 \%$ of CIE+. Physicians (PIE-) altered 52.9\% of CIE+; 12 of $168 \mathrm{CIE}+$ with imaging confirmed ASMI (7.1\%); 12 of 100 $\mathrm{PIE}+$ studies with imaging confirmed ASMI (12\%). In all 68 PIE-/CIE+, imaging was negative for ASMI.

Conclusion: ECG interpretation for prior ASMI by both physician and computer requires improvement. CIE for ASMI carries a high false-positive rate $(92.9 \%)$. In 68 PIE-/CIE+ ECG, imaging confirmed the absence of ASMI. Studies with a larger pool of data will be conducted to examine the trends identified. Further evaluation will be conducted to determine whether the false-positive CIE led to delays in surgical procedures, referrals for cardiology evaluation, additional testing, or undue patient emotional distress. Analysis of these ECG interpretation differences with modern artificial intelligence and machine learning techniques may lead to improved CIE algorithms.

\section{IMPLEMENTATION SCIENCE AND EMBEDDED RESEARCH}

OA2.01

\section{A Review of Reviews to Advance Understanding and Identify Strategies for Sustaining Evidence- Based Practices}

Sarah Birken, ${ }^{1}$ Emily Haines, ${ }^{1}$ Soohyun Hwang, ${ }^{1}$ David Chambers, ${ }^{2}$ Alicia Bunger, ${ }^{3}$ Michael Harrison, ${ }^{4}$ Per Nilsen ${ }^{5}$

${ }^{1}$ University of North Carolina at Chapel Hill, Chapel Hill, NC; ${ }^{2}$ National Cancer Institute, Bethesda, MD; ${ }^{3}$ Ohio State University, Columbus, $\mathrm{OH} ;{ }^{4}$ Agency for Healthcare Research and Quality, Rockville, MD; ${ }^{5}$ Linköping University, Linköping, Sweden

Background: Declining use of evidence-based practices (EBPs) following implementation is well documented. Sustained EBP use is critical for maintaining these practices' benefits over time; yet, understanding of EBP sustainment is limited. Limited understanding of sustainment may relate in part to lack of an agreed-on theory, model, or framework (TMF) to conceptualize sustainment. A highly relevant TMF could advance understanding and identify strategies for sustaining EBPs in health care systems. To identify potentially relevant sustainment TMFs, we evaluated the extent to which TMFs have been used, how TMFs have been used, and the relevance of the TMFs that have been used in extant sustainment research.

Methods: We drew upon recently published reviews of sustainment studies, evaluating 1) the frequency with which included articles used a TMF for conceptualizing sustainment, and 2) the relevance of TMFs for understanding sustainment using the Theory, Model, and Framework Comparison and Selection Tool, a user-friendly tool to help select TMFs used in implementation research.

Results: The 607 studies included across the 9 reviews cited 57 TMFs. Only 12 TMFs were used by more than 1 study. Of these, just 3 TMFs explicitly included sustainment as the outcome of interest; 9 offered face-valid explanations of proposed relationships; and 6 identified mechanisms through which included constructs influence sustainment. Only 3 TMFs (institutional theory, normalization process theory, organizational learning) performed adequately with respect to all these criteria.

Conclusion: TMFs are seldom used in extant sustainment studies. Of those that were used in sustainment studies, most TMFs were only used once, contributing to fractured understanding of sustainment. Of the more consistently used TMFs, few proposed face-valid relationships between included constructs and sustainment, limiting their ability to advance our understanding. Future research is needed to explore the TMFs that we identified as potentially relevant, as well as TMFs not identified in our study that have the potential to advance understanding of sustainment and identification of strategies for sustaining EBPs in health care systems. 
OA2.02

\section{Challenges of and Strategies Toward Optimal Implementation of Patient-Reported Outcome Measures for Clinical Care}

Jeanette Y. Ziegenfuss, ${ }^{1}$ Leif I. Solberg, ${ }^{1}$ Steve E. Asche,${ }^{1}$ Elizabeth Grossman, ${ }^{1}$ Christine K. Norton ${ }^{1}$

\section{${ }^{1}$ HealthPartners, Bloomington, MN}

Background: While patient-reported outcome measures (PROMs) have a history of use for public quality reporting, their use in the course/process of clinical care delivery at the individual patient level is newer and as such brings its own set of challenges. Clinical integration requires both that patients complete the measures and that providers use the data in the care of patients. Taking advantage of these data for research means facing the same challenges. If data are not addressed for a given patient due to either patient nonresponse or clinician nonuse, it detracts from future patient participation and diminishes researchers' ability to evaluate impact. Orthopedic centers in our care delivery system have been systematically collecting PROMs since spring 2017. The data has been available in Epic for use by clinicians since summer 2019.

Methods: In this work we describe current response rates to orthopedic PROMs in our care delivery system and use rates by clinicians. We describe efforts led by a multidisciplinary team with representation across research and clinical leadership to increase both patient response as well as clinicians' use of these data and the impact of these efforts.

Results: Patient methods to improve response rates include introduction of additional modes of survey delivery and introduction of measures developed with patient input. Clinician efforts included awareness building, staff support for use, and reporting of comparative use rates among clinicians. Preintervention patient response rates average about $60 \%$ depending on surgery type and time point. Preintervention clinician use rates were less than $2 \%$, suggesting a critical need for improvement. Early outcomes of these efforts, unavailable now, will be reported.

Conclusion: The use of PROMs to study and improve care delivery is dependent on high levels of engagement by both patients and clinicians. Working with operational care system leaders and patients, we identified and tested strategies to improve their use and value.

\section{OA2.03}

Implementation of an Opioid Withdrawal Clinical Pathway: A Health System Quality Improvement Initiative to Screen and Treat Medical Inpatients

Kimberly Williams, ${ }^{1}$ Beverly Wilson, ${ }^{1}$ Claudine Jurkovitz, ${ }^{1}$ Jo Melson, ${ }^{1}$ Jeffrey Reitz, ${ }^{1}$ Carmen Pal, ${ }^{1}$ Sherry Hausman, ${ }^{1}$ Erin Booker, ${ }^{1}$ Linda Lang, ${ }^{1}$ Terry Horton ${ }^{1}$

${ }^{1}$ ChristianaCare, Wilmington, DE
Background: Opioid-related inpatient hospital stays are increasing at alarming rates. Unidentified and poorly treated opioid withdrawal may be associated with inpatients leaving against medical advice and increased health care utilization. To address these concerns, we developed and implemented an opioid withdrawal clinical pathway to screen and treat medical service inpatients. This study aimed to evaluate the implementation and sustainability of this pathway in our health care system.

Methods: The pathway process included administration of a 2-item universal screening instrument to identify opioid withdrawal risk (ie, Opioid Withdrawal Risk Assessment [OWRA]), use of the validated Clinical Opiate Withdrawal Scale (COWS) to monitor opioid withdrawal symptoms and severity, and a 72-hour buprenorphine/naloxonebased treatment protocol. Process measures monitored our implementation outcomes, including adoption, fidelity, and sustainability of this new pathway model. To assess if there were changes in the knowledge, perceptions, and attitudes of participating nursing staff, a survey was administered to 4 hospital medical units before and after pathway implementation.

Results: Between December 2016 and December 2018, $72.4 \%(77,483$ of 107,071$)$ of admitted patients received the OWRA screening tool. Of those, $3.0 \%$ (2347 of 77,483) were identified as at risk for opioid withdrawal. Of those 2347 patients, 2178 (92.8\%) were assessed with COWS, and $29.6 \%$ of those (645 of 2178) were found to be in active withdrawal. Nearly half of patients in withdrawal (319 of $645,49.5 \%)$ were treated with buprenorphine/naloxone. More than half of nurses ( 83 of 145, 57.2\%) completed both the initial and follow-up surveys in February and December 2016, respectively. Pairs analysis of the pre/post survey data revealed that nurse respondents were significantly more confident in their ability to determine which patients are at risk for withdrawal $(\mathrm{P}=0.01)$ and had increased confidence in their ability to identify patients currently experiencing withdrawal $(\mathrm{P}=0.008)$.

Conclusion: Our study demonstrates a process for successfully implementing and sustaining a clinical pathway to screen and treat medical service inpatients for opioid withdrawal within the health care system. Standardizing care delivery for patients in opioid withdrawal also can improve nursing confidence when working with this complex population.

\section{OA2.04}

\section{Where Do I Store My Guns Until I'm Feeling Better? Evaluation of Outreach Methods for a Safe Storage Web-Based Decision Aid: Lock to Live}

Jennifer M. Boggs, ${ }^{1}$ LeeAnn M. Quintana, ${ }^{1}$ Arne Beck, ${ }^{1}$ Samuel B. Clinich, ${ }^{1}$ Laura Richardson, ${ }^{1}$ Amy Conley, ${ }^{1}$ James T. Hardee, ${ }^{1}$ Marian Betz ${ }^{2}$

${ }^{1}$ Kaiser Permanente Colorado, Denver, CO; ${ }^{2}$ University of Colorado, Aurora, $\mathrm{CO}$ 
Background: Safe storage of lethal means, such as firearms, medications, and other potentially dangerous household items, is a highly recommended suicide prevention practice. However, many patients with suicide risk are not counseled on lethal means safety in both medical and mental health settings. "Lock to Live" is an anonymously delivered webbased decision aid that incorporates patients' values and preferences into recommendations for safe storage of lethal means, specifically firearms. "Lock to Live" was designed with input from 60 stakeholders, including medical/mental health providers, public health researchers, and the firearm community, and had high acceptability in pilot testing. However, achieving adequate patient engagement is a major barrier for most web-based prevention strategies. We compared use of "Lock to Live" through a variety of delivery approaches (eg, email, mail) on website visit rates, with messaging that emphasizes anonymity.

Methods: Adult members of a large integrated health care system (Kaiser Permanente Colorado) who reported suicide risk on the Personal Health Questionnaire-9 depression screener, administered during mental health and medical visits, were invited to visit "Lock to Live." Invitation messages emphasized anonymity, and were co-designed with input from clinicians, public health researchers, and members of the Colorado Firearm Safety Coalition. Several different delivery method approaches were piloted: mail, text, email, patient medical record message, and providerdelivered. Two weeks after receiving an invitation to "Lock to Live," patients will receive a web-based survey to evaluate satisfaction, delivery method preferences, perceptions of privacy, ease of use, and safe storage behavior.

Results: Approximately 2500 patients will be invited to visit "Lock to Live" and complete a follow-up survey (500 per delivery method). Outreach started in October 2019 and will finish in March 2020. "Lock to Live" visit rates and survey data for each outreach approach will be reported.

Conclusion: Findings will be used to develop an implementation guide for "Lock to Live" that highlights advantages/ disadvantages for each delivery approach. Next steps are implementation of a large hybrid randomized effectiveness/ implementation trial of "Lock to Live" using 1 or more delivery approaches that are determined most acceptable to patients and feasible for implementation on a large scale.

\section{OA7.01}

Consequences of Using an Automated Electronic Health Record Tool to Screen and Enroll Participants in a Pragmatic Cluster-Randomized Hypertension Trial

Karen L. Margolis, ${ }^{1}$ A. Lauren Crain, ${ }^{1}$ Beverly B. Green, ${ }^{2}$ Patrick J. O'Connor, ${ }^{1}$ Leif I. Solberg, ${ }^{1}$ Mary Sue Beran, ${ }^{1}$ Anna Bergdall, ${ }^{1}$ Pamala A. Pawloski, ${ }^{1}$ Jeanette Y. Ziegenfuss, ${ }^{1}$ Amy Kodet, ${ }^{1}$ Daniel Rehrauer, ${ }^{1}$ Zeke
McKinney, ${ }^{1}$ Christine K. Norton, ${ }^{1}$ Patricia Haugen, ${ }^{1}$ Jeffrey Anderson, ${ }^{1}$ Benjamin Crabtree ${ }^{3}$ Nicole Trower, ${ }^{1}$ Deepika Appana, ${ }^{1}$ Rashmi Sharma, ${ }^{1}$ Sarah Norman, ${ }^{1}$ JoAnn Sperl-Hillen ${ }^{1}$

${ }^{1}$ HealthPartners, Bloomington, MN; ${ }^{2}$ Kaiser Permanente Washington, Seattle, WA; ${ }^{3}$ Rutgers Robert Wood Johnson Medical School, New Brunswick, NJ

Background: Pragmatic trials are desirable to demonstrate the efficacy of clinical interventions in real-world settings. They often apply minimal exclusion criteria to broadly accrue patients from usual care settings. However, these features may present challenges to intervention fidelity and adherence.

Methods: We enrolled patients into this clinic-randomized trial of clinic-based vs pharmacist-led telehealth-based blood pressure (BP) management using electronic health record (EHR) algorithms triggered by BP entry during primary care encounters. Algorithms applied the following criteria to screen patients for eligibility: 1) age $18-85$ years; 2) $>2$ hypertension diagnostic codes in 24 months; 3 ) an encounter with primary care provider in 12 months; 4) BP of $>150 / 95$ $\mathrm{mmHg}$ on two consecutive occasions; and 5) no identified pregnancy, end-stage renal disease, or hospice/nursing home residence. If eligible, a best-practice alert prompted rooming staff to pend a hypertension follow-up referral order, defaulted to nurse BP check in clinic-based and a pharmacist in telehealth-based clinics. Clinicians subsequently accepted, changed, or dismissed the order at their discretion. Patients were considered an enrolled participant when that order was signed, with a goal of 2000 enrollees.

Results: Of 222,134 patients screened over 18 months in 21 study clinics, $3796(1.7 \%)$ were eligible and 3072 (1.4\%, $81 \%$ of eligible) were enrolled. Enrollees (mean age: 60 years; sex: $47 \%$ male; race: $69 \%$ white; mean BP: $164 / 94$ $\mathrm{mmHg}$ ) differed slightly from eligible patients without signed follow-up orders (mean age: 63 years; sex: $51 \%$ male; race: $77 \%$ white; mean BP: 164/92 mmHg). Within 6 weeks of enrollment, $55 \%$ of clinic-based and $63 \%$ of telehealthbased enrollees attended hypertension follow-up, but only $32 \%$ of clinic-based and $27 \%$ of telehealth-based enrollees attended follow-up consistent with their randomized clinic assignment. Among the telehealth care subjects who saw the pharmacist, $86 \%$ agreed to home BP monitoring and $94 \%$ of those sent in at least 1 home BP measurement.

Conclusion: Automated EHR mechanisms to screen and enroll a high proportion of eligible patients into a pragmatic trial exceeded study enrollment goals. However, intervention exposure was lower than anticipated, particularly for subjects in telehealth-based clinics. The negative impact on intervention exposure and fidelity will potentially dilute intent-to-treat BP outcomes but will be assessed using a complementary per-protocol analysis. 
OA7.02

SUPPORT-AF II: A Cluster Randomized Study of Electronic Profiling and Messaging Combined With Academic Detailing for Providers Making Decisions About Anticoagulation in Patients With Atrial Fibrillation

Alok Kapoor, ${ }^{1}$ Azraa Amroze, ${ }^{1}$ Fatima Vakil, ${ }^{2}$ Sybil Crawford, ${ }^{1}$ Jacqueline Der, ${ }^{3}$ Jomol Mathew, ${ }^{1}$ Eric Alper, ${ }^{1}$ Dinesh Yogaratnam, ${ }^{1}$ Saud Javed, ${ }^{1}$ Rasha Elhag, ${ }^{1}$ Siddhartha Narayan, ${ }^{1}$ Donna Bartlett, ${ }^{1}$ Ahmed Nagy, ${ }^{1}$ Bevin Shagoury, ${ }^{4}$ Michael Fischer, ${ }^{5}$ Kathleen M. Mazor, ${ }^{1}$ Jane Saczynski, ${ }^{3}$ Jeffrey Ashburner, ${ }^{6}$ Renato Lopes, ${ }^{7}$ David McManus ${ }^{1}$

\section{${ }^{1}$ Reliant Medical Group/University of Massachusetts Medical School/Fallon Health, Worcester, MA; ${ }^{2}$ Nationwide Children's Hospital, Columbus, $\mathrm{OH}$; ${ }^{3}$ Northeastern University, Boston, MA; ${ }^{4}$ National Resource Center for Academic Detailing, Boston, MA; ${ }^{5}$ Brigham and Women's Hospital, Harvard Medical School, Boston, MA; ${ }^{6}$ Massachusetts General Hospital, Boston, MA; ${ }^{7}$ Duke University Medical Center, Durham, NC}

Background: Previous provider-directed electronic messaging interventions have not by themselves improved anticoagulation use in patients with atrial fibrillation (AF). Direct engagement with providers using academic detailing coupled with electronic messaging may overcome the limitations of the prior interventions.

Methods: We randomized outpatient providers affiliated with our health system in a 2.5:1 ratio to our electronic profiling/ messaging combined with academic detailing intervention. In the intervention, we emailed providers monthly reports of their anticoagulation percentage relative to peers for patients with $\mathrm{AF}$ who had elevated stroke risk $\left(\mathrm{CHA}_{2} \mathrm{DS}_{2}\right.$-VASc score of $\geq 2$ ). We also sent electronic medical record-based messages shortly before an appointment with an anticoagulationeligible but untreated AF patient. Providers had the option to send responses with explanations for prescribing decisions. We also offered to meet with intervention providers using an academic detailing approach developed based on knowledge gaps discussed in provider focus groups. To assess feasibility, we tracked provider review of our messages. To assess effectiveness, we measured the change in anticoagulation for patients of intervention providers relative to controls.

Results: We identified 85 intervention and 34 control providers taking care of 3591 and 1908 patients, respectively; 33 intervention providers participated in academic detailing. More than $80 \%$ of intervention providers read our emails, and $98 \%$ of the time a provider reviewed our in-basket messages. Replies to messages identified patient refusal as the most common reason for patients not being on anticoagulation $(11.2 \%)$. For the group of patients not on anticoagulation at baseline assigned to an intervention vs control provider, the adjusted percentage increase in the use of anticoagulation over 6 months was $5.2 \%$ vs $7.4 \%$, respectively ( $\mathrm{P}=0.21$ ).
Conclusion: Our electronic messaging and academic detailing intervention was feasible but did not increase anticoagulation use. Patient-directed interventions or provider interventions targeting patients declining anticoagulation may be necessary to raise the rate of anticoagulation.

\section{OA7.03}

Evaluation in a Continuously Learning Health System: Findings From a Critical Access Hospital Telemedicine Program

Meghan JaKa, ${ }^{1}$ Jennifer Dinh, ${ }^{1}$ Jeanette Y. Ziegenfuss, ${ }^{1}$ Jerome Siy, ${ }^{1}$ Ameet Doshi, ${ }^{1}$ Yonatan Platt, ${ }^{1}$ John Dressen ${ }^{1}$

\section{${ }^{1}$ HealthPartners, Bloomington, $M N$}

Background: Rigorous evaluation of novel clinical programs is a central tenant of continuously learning health systems. The triangulation of administrative health systems data and more traditional evaluation methods (eg, stakeholder surveys) is key in these efforts. The purpose of this work is to present findings from a robust evaluation of a new telemedicine program delivered in one health system's critical access hospitals. The specific evaluation presented herein tests the impact of telemedicine on stakeholder experience and clinical outcomes. Methods: This telemedicine program was designed to facilitate real-time overnight clinical care at critical access hospitals with an onsite care team and remote hospitalists via a hub-and-spoke model. Using a prospective pragmatic pre/ post evaluation design, administrative data (from electronic health records) was collected to measure clinical processes and outcome measures. Patient, hospitalist, and nurse surveys were collected throughout and after program implementation to understand impact on experience and make program improvements. Differences in clinical process measures were tested via chi-squared tests and logistic regression with a noninferiority test was used to compare longer-term clinical outcomes for telemedicine and usual care.

Results: Findings show that patients, nurses, and hospitalists would recommend the program and perceived a high quality of care, offering feasible recommendations to enhance communication. On average, lengths of hospital stay were shorter $(\mathrm{P}<0.01)$, and a higher proportion of care was delivered at night postimplementation $(\mathrm{P}=0.02)$. Telemedicine was demonstrated to be no worse than usual care in terms of hospital transfers (odds ratio: 0.73 ; 95\% CI: 0.54-1.00), but the evaluation was not able to demonstrate the same for other clinical outcomes.

Conclusion: This presentation will discuss how these evaluation findings were disseminated and used to strengthen this novel telemedicine program within the context of a learning health system. For example, these results were used to make practical improvements in program implementation and strengthen the case for future reimbursement of telemedicine in rural acute care. 
OA7.04

Results From a Randomized Trial Comparing Strategies for Helping Community Clinics Implement Guideline-Concordant Cardioprotective Care

Rachel Gold, ${ }^{1}$ Arwen Bunce, ${ }^{2}$ Stuart Cowburn, ${ }^{2}$ James V. Davis, ${ }^{1}$ Joanna Bulkley, ${ }^{1}$ Joan C. Nelson, ${ }^{1}$ Deborah J. Cohen, ${ }^{3}$ James W. Dearing, ${ }^{4}$ Michael Horberg ${ }^{5}$

${ }^{1}$ Kaiser Permanente Northwest, Portland, OR; ${ }^{2} \mathrm{OCHIN}$, Inc., Portland, OR; ${ }^{3}$ Oregon Health \& Science University, Portland, OR; ' ${ }^{4}$ Michigan State University, East Lansing, MI; ${ }^{5}$ Kaiser Permanente Mid-Atlantic States, Rockville, MD

Background: Statins can reduce cardiovascular disease risk in patients with diabetes mellitus, but statin prescribing lags behind current recommendations. We compared how 3 increasingly intensive implementation support strategies impacted community health centers' (CHCs) adoption of electronic health record (EHR) clinical decision support tools targeting guideline-concordant statin prescribing in diabetes mellitus. The tools (the "CVD Bundle") were adapted from a previously successful intervention.

Methods: In this mixed-methods, pragmatic comparative effectiveness trial, we randomized 29 CHCs with a shared EHR to 3 arms, which received varying implementation support: 1) implementation toolkit (CVD Bundle instructions, quality improvement techniques); 2) toolkit + in-person training with follow-up webinars; or 3) toolkit, training, webinars + offered practice facilitation. All study CHCs identified a champion to oversee research activities. Statin prescribing rates were compared across arms, and with those in 137 nonrandomized CHCs (a comparison group). We compared change in statin prescribing from 12 months preintervention through 36 months postintervention using adjusted Poisson regressions. We collected qualitative data using diverse methods (eg, interviews, observations).

Results: Statin prescribing increased preintervention to postintervention for all arms, although not in the hypothesized additive pattern. Arms 1 and 2 improved prescribing rates significantly (by $7 \%$ and $8 \%$, respectively) relative to comparison $\mathrm{CHCs}$ (3\%). Qualitative analysis identified interconnected clinic- and intervention-level factors underlying these results: 1) Problems with the EHR tools reduced their utility; 2) High staff turnover was a barrier to providing the planned implementation support; 3) The influence of study champions varied greatly; and 4) The provided support could not be adapted to each clinic's specific needs. Overall improvements in statin outcomes were likely influenced by a secular trend in awareness of statin prescribing guidelines and study participation bias.

Conclusion: The implementation strategies utilized were associated with small improvements in guideline-concordant statin prescribing. CHCs that received more intensive implementation support did not have greater improvements in prescribing rates. Efforts to implement care guidelines should ensure adaptability when providing implementation support and use early evaluations to determine the optimal form of such support for a given clinic. More research is needed on supporting change implementation, especially in underresourced settings like CHCs.

\section{P10.01}

\section{Validating Results From a Coincidence Analysis Using Diverse Stakeholders' Perspectives on Survivorship Care Plan Implementation in Complex Health Systems}

Sarah Birken, ${ }^{1}$ Soohyun Hwang, ${ }^{1}$ Rebecca Whitaker, ${ }^{2}$ Burcu Bozkurt, ${ }^{1}$ Sarah Asad, ${ }^{1}$ Tamara Huson, ${ }^{1}$ Ted James, ${ }^{3}$ Lawrence Shulman, ${ }^{4}$ Deborah Mayer ${ }^{1}$

${ }^{1}$ University of North Carolina at Chapel Hill, Chapel Hill, NC; '2Duke-Margolis Center for Health Policy, Duke University, Durham, NC; ${ }^{3}$ Beth Israel Deaconess Medical Center, Boston, MA; ${ }^{4}$ Abramson Cancer Center, University of Pennsylvania, Philadelphia, PA

Background: Achieving desired intervention outcomes requires successful implementation. Successful implementation requires identifying high-leverage strategies. Identifying high-leverage strategies in health systems is complicated because 1) multiple paths to successful implementation likely exist, and 2) perceptions of implementation success likely vary across stakeholders. These challenges can be addressed with coincidence analysis (CNA). CNA combines within-case and logic-based crosscase analysis to identify multiple "recipes" for successful implementation. Herein, we report a method for validating CNA results using the perspectives of the kinds of diverse stakeholders who characterize complex health systems. We use the example of applying CNA to identify strategies for the successful implementation of survivorship care plans (SCP), documents recommended to improve survivorship care.

Methods: We used data from in-depth, semi-structured interviews with 42 providers/staff in 39 cancer programs and 23 breast cancer survivors who received care in 15 of the 39 programs regarding SCP implementation success and strategies. We transformed codes from qualitative analyses into dichotomous or multivalue factors for use in CNA. We then created 2 datasets - 1 using the perspective of providers/ staff to define SCP implementation success and 1 using the perspective of survivors. We will analyze the 2 datasets independently using the R-package "can" function. Our final step will be to assess whether strategies for successful SCP implementation vary based on provider/staff vs survivor definitions of SCP implementation success.

Results: Four investigators analyzed data from 65 interviews over 5 weeks, representing more than 200 combined personhours of coding. Reconciling qualitative coding approaches to ensure intercoder reliability required 4 meetings before independent coding. Transforming qualitative codes into 
values for analysis in CNA effectively doubled the time required for coding. Similarly, CNA programming was required for 2 models, doubling the time typically required for analysis. We are concurrently comparing the model results.

Conclusion: If different perspectives on implementation success require divergent strategies, CNA's usefulness may be limited for achieving the goals of the diverse stakeholders who characterize complex health systems. However, if similar strategies predict outcomes from diverse perspectives, we would have strong evidence of CNA's reliability and thus its importance as a tool for identifying strategies for health systems change.

\section{P10.02}

\section{Applying User-Centered Design to Enhance the Usability and Usefulness of Patient-Reported Outcome Measures: A Case Example in Adolescent and Young Adult Oncology}

\author{
Emily Haines, ${ }^{1}$ Alex Dopp, ${ }^{2}$ Byron Powell, ${ }^{3}$ Angela \\ Stover, ${ }^{1}$ Andrew Smitherman, ${ }^{1}$ Lauren Lux, ${ }^{1}$ Sarah \\ Birken $^{1}$ \\ ${ }^{1}$ University of North Carolina at Chapel Hill, Chapel \\ Hill, NC; ${ }^{2}$ RAND Corporation, Santa Monica, CA; \\ ${ }^{3}$ Washington University in St. Louis, St. Louis, MO
}

Background: The National Cancer Institute, National Institutes of Health, and others increasingly advocate for the use of patient-reported outcome measures (PROMs) in practice to better address needs of patients with cancer. However, PROMs are unlikely to address patient needs if they lack usability (ie, patients/providers find them difficult to use) or if they are not linked to available services/resources that will address identified needs (ie, have low usefulness). To facilitate the delivery of services/resources to address the unmet needs of adolescents and young adults (AYAs) with cancer, we developed the Needs Assessment \& Service Bridge (NA-SB). To optimize the usability and usefulness of NA-SB, we leveraged user-centered design, an iterative process for intervention development based on prospective user engagement.

Methods: We solicited input from a design team of users and researchers throughout the project. We used mixed-methods usability testing to collect data on user interactions with an existing PROM, including an online survey and cognitive interviews with AYAs and concept mapping with providers, to map PROM-identified needs onto services/resources. We then used ethnographic methods with prospective users to identify contextual factors to consider in the design and implementation of NA-SB; we analyzed ethnographic data using template analysis. Finally, through two design team workshops, we designed a useful and usable NA-SB prototype and implementation plan.

Results: We will report descriptive statistics on users as well as usability testing and ethnography results. We will describe how we collaborated with the design team to produce an NA-SB prototype and implementation plan as well as discuss the viability of user-centered design for optimizing PROM usability and usefulness in other disease contexts.

Conclusion: PROMs are unlikely to be implemented if users find them difficult to use or irrelevant. Even if implemented successfully, a PROM's usefulness will be limited without an explicit link between PROM-identified needs and available services/resources to address their needs. In this case example, we leveraged user-centered design to enhance an existing PROM's usability and usefulness, accounting for context to promote its receptivity and anticipate needed implementation strategies - in effect, harmonizing intervention, context, and implementation to promote NA-SB's implementation and effectiveness. Future efforts should apply user-centered design to enhance the role of PROMs in improving patient outcomes.

\section{P10.03 \\ CM-SHARE Spread: Scaling Up a Digital Health
Research Pilot}

Hannah Husby, ${ }^{1}$ Jake Delatorre-Reimer, ${ }^{1}$ Farah Refai, ${ }^{1}$ Xiaowei (Sherry) Yan, ${ }^{1}$ J.B. Jones ${ }^{1}$

${ }^{1}$ Sutter Health, Walnut Creek, CA

Background: Digital health tools have the potential to play an integral role in transforming health care. Health care systems are uniquely positioned to understand how to implement these tools in real-world settings, although the path from pilot tests to systemwide dissemination remains challenging. We describe our experience with CM-SHARE, a web-based digital health application designed to help physicians and patients manage cardiometabolic (CM) conditions. In its pilot, CM-SHARE demonstrated successful adoption and led to statistically significant improvements in measures of physician efficiency. Our objective was to then spread CMSHARE across our health care system.

Methods: We conducted a nonsystematic assessment of available toolkits for dissemination and selected 3 sets of strategies that addressed anticipated challenges in our system. Our people strategies included engaging the right level of leadership to endorse and support the spread effort and identifying local champions for day-to-day support. Our communications strategies included identifying the relevant meetings to engage and inform future users and creating training and informational materials. Our resources strategies focused on ensuring appropriate technical modifications were made to the pilot and making sure day-to-day support was available in individual clinics. We are testing these methods in a stepwise fashion and will modify based on learnings before expanding to other medical foundations and the rest of the system.

Results: We identified 2 quality leaders to endorse the systemwide spread effort. We partnered with a physician 
champion during an 8-month "pre" period to garner support among Sutter Health leaders across stakeholder groups. Sutter's electronic health record trainers provide side-byside support to practicing clinicians. This train-the-trainer approach allows for a level of day-to-day support beyond what research could provide alone. We developed training materials and flyers to communicate about the purpose, value, and availability of the tool. Feedback from users is systematically collected by the trainer, and appropriate modifications will be communicated to users. Our poster will describe adoption rates among new users, effective methods and strategies of communication, and key physician feedback. Conclusion: By engaging physician champions and leadership, the research team has successfully initiated a systemwide dissemination effort for a new digital health tool. Findings will be applied to future spread sites.

\section{P10.04} Testing the Effectiveness of an Employee-Offered
Behavioral Weight-Loss Program in a Learning
Health System: Study Design and Baseline Results

\author{
Meghan JaKa, ${ }^{1}$ Jennifer Dinh, ${ }^{1}$ Rachael Rivard, ${ }^{1}$ \\ Stephen Herrmann, ${ }^{2}$ Nico Pronk, ${ }^{1}$ Jeanette Y. \\ Ziegenfuss, ${ }^{1}$ Joel Spoonheim ${ }^{1}$
}

${ }^{1}$ HealthPartners, Bloomington, MN; ${ }^{2}$ Sanford Health, Sioux Falls, SD

Background: Obesity is a leading cause of the most expensive chronic health conditions faced by employers. As such, learning health systems aim to test and offer effective weight-loss programs as a part of well-being incentive programs. This presentation reports one health plan's efforts to pilot and test the effectiveness of an evidence-based behavioral weight-loss program among its employees, providing a unique opportunity to combine employeereported, claims, and programmatic data.

Methods: This pragmatic pre/post evaluation measures fidelity, outcomes (psychosocial, lifestyle, workplace, health, well-being), and incentive program preferences via survey, claims, and programmatic data. Employees/dependents with insurance through a large health system in the Midwest were offered "Profile by Sanford" at a discounted rate. Profile includes coaching, meal replacement offerings, and regular self-monitoring (food journals and self-weighing). Profile participants were recruited to the study immediately following enrollment. Surveys are completed at baseline, 3, 6, and 12 months, and claims data are pulled for the year before and after enrollment. Descriptive statistics will be presented for measured baseline outcomes. Designed intent-to-treat and per-protocol analysis plans also will be presented.

Results: Of the 354 employees/dependents enrolled in Profile, $41 \%$ provided consent for the study and completed baseline measures. Study participants (mean age: $50 \pm 10$ years; mean body mass index: $33 \pm 6 \mathrm{~kg} / \mathrm{m}^{2}$ ) were primarily female $(90 \%)$ and of non-Hispanic white race $(95 \%)$ and had at least some college education (80\%). Nearly all $(96 \%)$ reported prior weight-loss attempts. Baseline life and job satisfaction was high $(81 \%$ and $93 \%$, respectively, said $7+$ on a 10 -point scale), with more moderate levels of healthy weightrelated behaviors (64\% avoid sugar-sweetened beverages, $21 \%$ eat $5+$ fruits/vegetables daily, $12 \%$ with $5+$ days/week of vigorous activity). Preliminary fidelity results indicate initial satisfaction with Profile was high ( $\mathrm{n}=42$ provided unprompted positive feedback in open-ended questions), with some offering opportunities for improvement (eg, related to technology, coaching).

Conclusion: Findings suggest the feasibility of implementing and evaluating a weight-loss program offered to employers as an incentivized health and well-being offering. The results of this study will be discussed in the context of other weightrelated offerings and will be used to make programmatic improvements and informed health plan decisions, following best practice in learning health systems.

\section{P10.05}

Utilizing Dissemination Strategies to Implement a Systemwide Initiative in a Large Health Care System

Farah Refai, ${ }^{1}$ Xiaowei (Sherry) Yan, ${ }^{1}$ Zijun Shen, ${ }^{1}$ William Isenberg, ${ }^{1}$ Mitesh Patel, ${ }^{2}$ Amol Navathe, ${ }^{2}$ Kit Delgado, ${ }^{2}$ Rebecca Pepe ${ }^{2}$ Joshua Liao ${ }^{3}$

${ }^{1}$ Sutter Health, Walnut Creek, CA; ${ }^{2}$ University of Pennsylvania, Philadelphia, PA; ${ }^{3}$ University of Washington, Seattle, WA

Background: Systemwide interventions are challenging to disseminate effectively across large health care systems. Sutter Health, a large health care system in Northern California, in collaboration with University of Pennsylvania and University of Washington, implemented a 6-month quality improvement initiative across all emergency departments (EDs) and urgent care clinics (UCs) to reduce opioid prescriptions. We describe our experience using dissemination strategy and communication methods to navigate and successfully implement this intervention in all 21 EDs and 27 UCs across Sutter.

Methods: We assessed strategies available from dissemination science and selected 2 sets that directly addressed anticipated challenges disseminating in both UCs and EDs. We focused on communications and people. Our top-down communications approach comprised establishing 3 primary levels of communication to engage Sutter leaders and use their influence to enroll clinicians to the initiative - first level: CEOs; second level: physician leadership; and third level: medical directors. Our people strategy centered on engaging influential stakeholders to support and endorse systemwide dissemination. We tested these strategies in a pilot phase involving 6 clinicians and implemented them in all EDs and UCs after successful results. 
Results: Every ED and UC in the system, which included 451 eligible clinicians, was successfully enrolled in the initiative. Our team found that EDs and UCs are managed very differently at Sutter, and as such the top-down communications strategy worked best for EDs and the people strategy for UCs. Structurally, all Sutter ED leaders and sites are managed by the system ED leadership group, and endorsement from the chair and group is key to successful dissemination to all sites. In contrast, Sutter UCs practice independently from one another. UCs required significant time and effort communicating directly with each medical director and developing site-specific presentations to obtain support. Our poster will describe effective strategies of communication and key learnings for UC and ED dissemination.

Conclusion: Reliable communication methods and leadership buy-in are key for universal, systemwide dissemination efforts. An understanding of work culture and identification of key stakeholders and gatekeepers is crucial for efficient and successful dissemination.

\section{P10.06}

\section{Using Practice-Based Evidence to Optimize Survivorship Care: Results From a Pilot Test of Supporting Transitions AfteR Treatment (START)}

\section{Sarah Birken, ${ }^{1}$ Burcu Bozkurt, ${ }^{1}$ Octavia Gladden, ${ }^{1}$ MaryBeth Grewe, ${ }^{1}$ Emily Haines, ${ }^{1}$ Soohyun Hwang, ${ }^{1}$ Ann Jackson, ${ }^{2}$ Darren DeWalt, ${ }^{3}$ Deborah Mayer, ${ }^{1}$ Morris Weinberger, ${ }^{1}$ Sarah Nikolich, ${ }^{1}$ Karen Kimel-Scott, ${ }^{1}$ Jennifer Leeman ${ }^{1}$}

${ }^{1}$ University of North Carolina at Chapel Hill, Chapel Hill, NC; 'lead patient partner; ${ }^{3}$ UNC School of Medicine, Chapel Hill, NC

Background: After completing treatment, many low-risk breast cancer survivors receive follow-up care from oncology providers despite the benefits of receiving follow-up care from primary care providers (PCPs). Interventions intended to promote survivors' transitions from oncology providers to PCPs exist, but their effectiveness is limited. We addressed the limitations of extant interventions by developing Supporting Transitions AfteR Treatment (START), a 3-step, low-intensity intervention specifically targeting the multilevel barriers to transitioning survivors to primary care.

Methods: In this study, we describe refining START based on the demands of a complex practice environment, pilot testing it in a tertiary academic comprehensive cancer program with the objectives of integrating START into existing clinic processes. Currently, we are conducting interviews with oncology providers, survivors, and PCPs regarding START's appropriateness, acceptability, and feasibility.

Results: The oncology provider enrolled in the pilot study identified several opportunities to refine START based on the demands of a complex practice environment. Refinements included increasing the flexibility of oncology provider education, reminding oncology providers of upcoming visits for potentially eligible survivors, and using an existing clinic form to deliver START content. In preparation for implementing the refined START intervention, we identified 6 potentially eligible survivors (14\%) among 43 scheduled patients across 5 clinic days.

Conclusion: Our careful attention to incorporating lessons from efforts to change clinical practice in the context of complex practice environments into START distinguishes our intervention from extant survivorship care improvement interventions, which have largely been based on expert consensus and have not been refined to reflect the challenges of implementation in the context of complex practice environments and thus have had substantial difficulty with implementation. By incorporating practice-based evidence into START, this study meaningfully contributes by generating practice-based evidence regarding an intervention intended to optimize survivorship care.

\section{P10.07}

Approaches Used to Develop a Clinic-Based Medication Disposal Program Targeting OpioidNaïve Patients

\author{
Lorraine Tusing, ${ }^{1}$ Jove Graham, ${ }^{1}$ Alanna Kulchak \\ Rahm, ${ }^{1}$ Eric Wright ${ }^{1}$
}

\section{${ }^{1}$ Geisinger Health, Danville, PA}

Background: Opioid-related drug overdose deaths continue to rise, with unused prescription opioids being a primary entry point. Evidence suggests that education by providers and access to disposal items improves disposal rates among patients with unused opioids but is not widely implemented. The goal of our larger project is to develop and evaluate the effectiveness and scalability of a clinic-based medication disposal program for patients newly prescribed opioid medications utilizing the RE-AIM framework to guide implementation planning and evaluation. Herein, we describe the processes used to develop the program and the impact of these approaches on the pragmatic nature of the study.

Methods: The initial medication disposal program was developed by the study team, inclusive of a literature search, informal discussion with clinic leadership, and institutional experience with implementing programs into primary care. This program design was reviewed and critiqued through 1:1 unstructured interviews with system leaders, clinicians, embedded service line best practice team representatives, information technology support leaders, and clinical informaticians. The PRagmatic Explanatory Continuum Indicator Summary (PRECIS-2) was used to evaluate the pragmatic level of the larger evaluation study design before and after adjustments based on stakeholder feedback.

Results: 13 primary care clinics were identified for participation. Initial design involved physicians providing both written and verbal education, along with physical 
disposal items (eg, a mail-back disposal bag) at the clinic visit, but stakeholders expressed concerns over poor adoption of and fidelity to this process. Following input from stakeholder interviews, program design was revised to remove the physical supply of written education materials and disposal items from the clinic to a centralized location and the mailing of materials directly to the patient by the study team. This resulted in a slightly less pragmatic design (driven by the movement of organization from very pragmatic to rather pragmatic due to the insertion of a centralized mail process). The PRECIS-2 results for the finalized overall study design in each category ranged from rather pragmatic (4) to very pragmatic (5).

Conclusion: Following interviews with clinical and system leadership, the medication disposal program to be implemented and evaluated remained pragmatic and is expected to reduce barriers to effective implementation.

\section{P10.08}

Evaluation of a Pilot Implementation of PopulationBased Risk Assessment for Breast Cancer Screening and Prevention

\author{
Dina Hassen ${ }^{1}$ Alanna Kulchak Rahm, ${ }^{1}$ Susan R. \\ Snyder, ${ }^{2}$ Mallory Snyder, ${ }^{1}$ Brianna Maciejewski, ${ }^{1}$ \\ Serena Tripp,$^{1}$ Jason Brown, ${ }^{1}$ Rosemary Leeming, ${ }^{1}$ \\ Aalpen Patel ${ }^{1}$ Jing $\mathrm{HaO}^{1}$
}

${ }^{1}$ Geisinger Health, Danville, PA; ${ }^{2}$ Georgia State University, Atlanta, GA

Background: Risk stratification is increasingly recommended as the new standard of care to identify and offer appropriate breast cancer screening and prevention services. Beginning in May 2018, Geisinger Medical Center in Danville, Pennsylvania, implemented a pilot population-based risk assessment strategy for all women receiving routine screening mammograms in Radiology. Language identifying patients' risk of either "increased" or "not increased over that of other women your age" was incorporated into mammography reports. A recommendation for referral to the High-Risk Breast Clinic (HRBC) for more personalized risk evaluation and appropriate risk-based preventive care management was placed in the mammography report for women identified at increased risk. The study objective is to evaluate the initial outcomes of this pilot implementation to determine if it impacted referral and use of evidence-based services.

Methods: We conducted a retrospective analysis on women who underwent routine screening mammography at Geisinger Medical Center and identified as at increased risk during the first 6 months of the risk assessment pilot implementation. The patients' risk-level category was identified using chart review, and health care utilization was obtained from the electronic health record. We will identify retrospectively a comparison cohort of women who underwent routine screening mammography at other
Geisinger facilities without the implementation during the same time period. The comparison cohort will be obtained by matching using a 1:1 ratio on the bases of age, breast density, Gail score, and comorbidities. We will measure the percentage of increased-risk women identified. Descriptive analysis will be conducted to compare the two cohorts on referral to HRBC and their postmammography guidelinerecommended preventive screening and prevention services at 1-year preimplementation and at 1-year postimplementation. Difference-in-differences will be applied to evaluate these initial outcomes of risk assessment implementation.

Results: Preliminary data show that during the first year of implementation, about 7400 women underwent routine screening mammography with risk assessment. Using data from the first few weeks postimplementation, about $65 \%$ of women were identified as being at increased risk for breast cancer.

Conclusion: Study findings on the initial impact of the risk assessment pilot are expected to demonstrate its effectiveness and areas for improvement for achieving personalized breast cancer screening and prevention.

\section{P10.09}

Barriers and Facilitators to Genetic Testing for Familial Hypercholesterolemia in the United States

\author{
Rachele Hendricks-Sturrup, ${ }^{1}$ Kathleen M. Mazor, ${ }^{2}$ \\ Amy Sturm, ${ }^{3}$ Christine Y. Lu ${ }^{1}$
}

${ }^{1}$ Harvard Pilgrim Health Care, Boston, MA; ${ }^{2}$ Reliant Medical Group/University of Massachusetts Medical School/Fallon Health, Worcester, MA; ${ }^{3}$ Geisinger Health, Danville, PA

Background: Familial hypercholesterolemia $(\mathrm{FH})$ is an underdiagnosed condition in the United States and globally. It is a genetic risk factor for premature cardiovascular disease, is responsible for an estimated 600,000 to 1.2 million preventable vascular events, and affects an estimated 1 in 250 individuals in the United States. FH genetic testing can identify a causal gene variant in $60 \%$ to $80 \%$ of clinically suspected $\mathrm{FH}$ cases. Clinical FH genetic testing, however, is underutilized in the United States for reasons that are poorly understood.

Methods: We searched the PubMed/MEDLINE database and 7 peer-reviewed journals to identify potential barriers and facilitators to $\mathrm{FH}$ genetic testing in the United States. After filtering 2340 articles based on our inclusion criteria, we selected 9 commentaries or expert opinions and 8 empirical studies reported from January 2014 to March 2019 for inclusion.

Results: After applying the Consolidated Framework for Implementation Research (CFIR) to understand the nature of these potential barriers and facilitators, we identified a total of 26 potential barriers and 15 potential facilitators (estimated barrier-to-facilitator ratio of 1.73). We estimated ratios of 
potential barriers to facilitators were calculated for each CFIR domain (characteristics of intervention, outer setting, inner setting, characteristics of individuals, and process).

Conclusion: Findings derived from our systematic approach to the literature and calculations of estimated baseline ratios of barriers and facilitators can be used to inform future research on understanding implementation of genetic testing for familial hypercholesterolemia in the United States.

\section{ELECTRONIC HEALTH RECORDS, TECHNOLOGY, AND DIGITAL HEALTH}

\section{OA3.01 \\ Patient Perceptions of a Web-Based Medication Reconciliation Tool}

\author{
Michael Gionfriddo, ${ }^{1}$ Vanessa Duboski, ${ }^{1}$ Melissa Kern, ${ }^{1}$ \\ Eric Wright ${ }^{1}$
}

${ }^{1}$ Geisinger Health, Danville, PA

Background: Despite routine review of medication lists during patient encounters, patients' medication lists remain incomplete and not reflective of actual medication use. Reconciling medication use from existing health records, along with external locations (eg, pharmacies, other provider/hospital records and care facilities) remains a large challenge. Advances in interoperability and digital connections provide a foundation for integration of these once-disparate information sources. We developed a webbased tool $\left(\mathrm{MedTrue}^{\mathrm{TM}}\right)$ that facilitates the reconciliation of these varying sources of information and places it into the use of patients and providers through direct electronic health record integration. We report on patient satisfaction, usability, and acceptability of the tool.

Methods: We conducted a mixed-methods study of patient experiences with MedTrue through a patient-facing survey and through semi-structured interviews. The survey assessed patient satisfaction with the tool and consisted of 5 questions. A study team member conducted semi-structured interviews with a convenience sampling of patients following clinic encounters at which MedTrue was used. The interviews lasted up to 30 minutes, and patients were offered a $\$ 10$ gift card for participating. Interviews continued until it was decided that data saturation had been met. Interviews were transcribed verbatim and thematically analyzed.

Results: MedTrue was used by 7341 patients; 1450 (20\%) completed the optional patient satisfaction survey at the conclusion of MedTrue. Patients were satisfied with MedTrue, giving it an average rating of 8 out of 10 . Additionally, $88 \%$ agreed or strongly agreed that it was easy to use, and $85 \%$ agreed or strongly agreed that it helped them create a more accurate medication list. We recruited 14 patients (and 1 spouse) for semi-structured interviews.
Most patients found it easy to use and were able to complete the tool quickly. While some patients felt the tool made the visit more efficient, other patients felt that it created unnecessary work. Helpful features included the ability to visually see their list as well as the ability to add and remove medications, which patients felt improved the accuracy of their medication list.

Conclusion: A web-based tool to facilitate medication reconciliation was well received by patients.

OA3.02

Leveraging Electronic Health Record Data and Partnerships to Enhance Latent Tuberculosis Infection Surveillance

Jon Puro, ${ }^{1}$ Tracy Ayers, ${ }^{2}$ Melanie Canterbury, ${ }^{3}$ Thomas Carton, ${ }^{3}$ Elizabeth Crull, ${ }^{3}$ Teresa Schmidt, ${ }^{1}$ Jonathan Todd, ${ }^{4}$ Laura Vonnahme, ${ }^{2}$ Jee Oakley, ${ }^{1}$ Amy Zlot $^{1}$

${ }^{1}$ OCHIN, Inc., Portland, OR; ${ }^{2}$ Centers for Disease Control and Prevention, Atlanta, GA; ${ }^{3}$ Louisiana Public Health Institute, New Orleans, LA

Background: Eliminating tuberculosis (TB) in the United States requires correctly identifying and treating patients with latent tuberculosis infection (LTBI). Appropriate screening and interventions require a robust surveillance system capable of measuring screening, treatment, and risk factors, particularly in primary care settings. Community health centers are instrumental, as they see a large proportion of people who may be at higher risk for LTBI.

Methods: We conducted secondary data analysis utilizing Big Data from a large U.S.-based electronic health record (EHR) network with clinics in 20 states. Initial epidemiological exploration of LTBI/TB cascade of care will inform future interventions using EHR tools to identify and treat patients with LTBI/TB. The population studied included 2,190,686 low-income and underserved patients seen in approximately 700 safety-net clinics between January 1, 2012, and December 31, 2016.

Results: This study calculates the proportions of patients screened for LTBI/TB using 3 separate TB screening tests: tuberculin skin tests (TST), QuantiFERON-TB Gold (QFT), and T-SPOT.TB. Among the study cohort, $151,195(6.9 \%)$ unique patients had a total of 185,854 TB screening tests. Most screening tests were TST, with a total of $139,561(75.1 \%)$, followed by 45,567 (24.5\%) QFT and 726 T-SPOT.TB $(0.4 \%)$.

Conclusion: Using Big Data systems for TB surveillance is challenging, especially finding and normalizing pertinent lab data for LTBI and TB and highly variable medicationdispensing data. Many opportunities exist to improve data collection in primary care setting in order to enhance LTBI and TB surveillance. Next steps include adapting data extraction techniques to be utilized in The National PatientCentered Clinical Research Network $\left(\right.$ PCORnet $\left.^{\mathbb{R}}\right)$. 
OA3.03

\section{Predicting Sepsis Using Machine Learning: Impact of Design Decisions on Assessment of Predictive Performance}

Sylvia Sudat, ${ }^{1}$ Philippe Boileau, ${ }^{1}$ Shruti Vaidya, ${ }^{1}$ Ridhima Nerlekar, ${ }^{1}$ Alexandra Scott, ${ }^{1}$ Alice Pressman ${ }^{1}$

\section{${ }^{1}$ Sutter Health, Walnut Creek, CA}

Background: Machine learning-based predictive modeling has been increasingly explored and implemented for various health-related applications. In these settings, premodeling design decisions may be complex and heavily influence both a model's performance and its potential clinical utility. Traditionally, little attention is paid to the possible impact of these design decisions. Sepsis has been a focus of early identification efforts using predictive modeling. Sepsis is also difficult to define - its clinical definition is changing over time - and those who meet clinical criteria may or may not ultimately develop sepsis. It can be nearly impossible to distinguish appropriate identifications (sepsis averted due to clinical intervention) from true false positives. Sepsis treatment is also highly time-sensitive, making the choices regarding handling of time-dependent data, such as lab results and vital signs, very important in terms of the model's utility. We present the results of a project using machine learning to predict onset of sepsis and describe the impact of modeling design decisions on assessed performance.

Methods: We studied 768,955 adult hospitalizations from 2014 to 2018 at 23 acute care hospitals within Sutter Health, a large health system in northern California that offers hospital-based and ambulatory care. We used data during hospitalization, prior-year comorbidities, and preadmission clinical/utilization data over prior 2 weeks. We applied machine learning methods to predict sepsis using standard clinical criteria and plan to further assess alternate definitions of sepsis onset and the predictive utility of preadmission data. Results: Early results predicting sepsis onset using the first 2 hours of hospitalization data produced areas under the receiving operating curve of $0.877,0.855$, and 0.843 for developing sepsis within the subsequent 2, 4, and 6 hours, respectively. More than $70 \%$ of those meeting sepsis criteria did not have a sepsis discharge diagnosis, and future analyses will explore how the decision to treat these individuals as true or false positives influences model performance and utility.

Conclusion: Machine learning-based predictive modeling is an increasingly attractive way to utilize large amounts of electronic health record data to identify individuals with increased risk or need for health care services. Careful attention must be paid not only to standard model performance measures, but also to the impact of premodeling design decisions.
OA3.04

Adding Unique Device Identifiers to Claims at Two Health Systems: 2-Year Results

Jove Graham, ${ }^{1}$ Dan Krupka, ${ }^{2}$ Amanda Reich, ${ }^{3}$ Angela $\mathrm{Li}^{4}{ }^{4}$ Kevin Capatch, ${ }^{1}$ Joel Weissman ${ }^{5}$

${ }^{1}$ Geisinger Health, Danville, PA; ${ }^{2}$ Twin Peaks Group, LLC, Lexington, MA; 'BRrigham and Women's Hospital, Boston, MA; ${ }^{4}$ Blue Cross Blue Shield of Massachusetts, Boston, MA; ${ }^{5}$ Partners HealthCare, Somerville, MA

Background: Safety surveillance is difficult for medical devices, partly because unlike medications that can be tracked on insurance claims via National Drug Code numbers, no similar system has existed for devices until recently. In 2014, the U.S. Food and Drug Administration (FDA) began requiring manufacturers to label medical devices with unique device identifiers (including device identifiers [DIs]). In advance of expected 2020 regulations that would add DIs to claims, Partners HealthCare, Blue Cross Blue Shield of Massachusetts, and Geisinger Health collaborated to demonstrate feasibility of transmitting DIs from catheterization laboratories to insurance claims, then analyzed the resulting claims.

Methods: At Partners, devices were scanned and DIs transferred to billing via the electronic health record (EHR) using an extension rule to write to the claim when appropriate. At Geisinger, DIs were sent by inventory management software to billing, then mapped to the claim form. We examined claims from 2017 to 2019 to categorize devices by FDA product code and analyzed 90-day outcomes, including all-cause readmissions, emergency department visits, myocardial infarction, revascularization, renal failure, and bleeding. We stratified results by whether the hospitalization occurred within the same or a different health system than where the initial procedure was performed, an important information advantage of insurance claims.

Results: At Geisinger, 1493 devices for 489 patients were transmitted to claims; at Partners, 888 devices for 463 patients were transmitted. The most frequently used implants were coronary drug-eluting stents ( $\mathrm{n}=213$ patients). Of these 213 drug-eluting stent patients, 45 (21\%) had a 90-day hospital readmission, $26(58 \%)$ of which occurred outside the initial health system and therefore appeared in claims but would not have appeared in the original hospital's EHR.

Conclusion: Generating insurance claims with DIs in two health systems provided proof of concept of the feasibility of adding DIs to claims with minimal work beyond what is required for other routine information system updates. Claims provide meaningful advantages to EHRs in that they capture out-of-system postprocedure events. For health system analysts and researchers who use claims data, having detailed information on the manufacturer and model of implanted devices that can be linked to patient outcomes will be a significant step forward toward improved device safety. 
P16.01

Evaluation of Implementing Electronic Prior Authorization for Medications in a Large Health Care System

Cheryl Stults, ${ }^{1}$ Xiaowei (Sherry) Yan, ${ }^{1}$ Satish Mudiganti, ${ }^{1}$ Mengdong $\mathrm{He}^{2}$ Lisa Dean-Gilley, ${ }^{1}$ Luke Forster-Broten, ${ }^{3}$ Nicholas Webber, $^{3}$ Julie Lauffenburger, ${ }^{2}$ Michael Fischer ${ }^{2}$

\section{${ }^{1}$ Sutter Health, Walnut Creek, CA; ${ }^{2}$ Brigham and Women's Hospital, Boston, MA; ${ }^{3}$ Surescripts, Arlington, VA}

Background: Prior authorization is intended to manage prescription drug use and spending by channeling medication use to patients who meet prespecified criteria to receive selected medication. However, in practice, prior authorization is not only imposing substantial administrative burden to physicians, it also is leading to delays in prescription filling that may delay patients from receiving potentially beneficial medications. Electronic prior authorization (ePA) is an electronic solution that automates the process of prior authorizations in real time by contacting the pharmacy benefit manager (PBM). We discuss the implementation and impact of an ePA application embedded into the Epic electronic health record in a large health care delivery system.

Methods: Sutter Health is an integrated health care system in Northern California serving more than 3 million patients annually. Sutter implemented ePA in a staggered roll-out from March 2018 to November 2018. Summary statistics were conducted to understand how ePA is utilized following full implementation. "Resolved" status means that ePA was approved or denied by the PBM.

Results: From December 1, 2018, to June 30, 2019, ePA was queried 59,394 times. Of these, 23,953 (40.33\%) were resolved with an approved $(n=15,494)$ or denied $(n=8459)$ status. The departments with the most ePA-resolved requests were family medicine $(\mathrm{n}=9823)$, internal medicine $(\mathrm{n}=4568)$, dermatology $(\mathrm{n}=1380)$, rheumatology $(\mathrm{n}=1136)$, and endocrinology $(n=840)$. The 5 most common therapeutic classes with resolved ePA were dermatological/anorectal/ mouth-throat/dental/ophthalmic/otic $(\mathrm{n}=5559)$, analgesic/ anti-inflammatory/migraine/gout agents/anesthetics $(n=4273)$, cardiovascular agents $(n=2679)$, endocrine and metabolic agents $(\mathrm{n}=2094)$, and antihistamines/nasal agents/ cough \& cold/respiratory/miscellaneous $(n=1982)$. The average time between ePA initiation and resolved was 44.47 hours (median: 14.23 hours), with a range of 0 to 5106.63 hours. The therapeutic subclass with the shortest time to resolved was dermatological/anorectal/mouth-throat/dental/ ophthalmic/otic (mean: 38.96 hours, median: 8.42 hours), and the longest was cardiovascular agents (mean: 56.61 hours, median: 18.08 hours).

Conclusion: To our knowledge, this is the first academic report on the evaluation of ePA in real-world practice settings. Initial results suggest that most resolved ePAs were from family and internal medicine departments and from the therapeutic classes of dermatological/anorectal/mouth-throat/ dental/ophthalmic/otic and analgesic/anti-inflammatory/ migraine/gout agents/anesthetics. Further coordination is necessary to work with PBMs to improve the resolution rate.

\section{P16.02 \\ Exploring Healthy Eating and Digital Health Care Support in an Overweight Sample of Working Rural Women}

Sharon S. Laing ${ }^{1}$

${ }^{1}$ University of Washington Tacoma, Tacoma, WA

Background: Residents in rural communities in the United States have increased probability of being obese. Research show barriers to healthy eating. Technology, including mobile-based tools, can provide healthy eating coaching and social connection to disperse communities. This study explored healthy eating and technology practices related to preventive care behaviors in a sample of working rural women who self-identify as overweight/obese.

Methods: 60 women completed a 32-item survey that assessed Centers for Disease Control and Prevention (CDC)recommended fruit and vegetable $(\mathrm{F} / \mathrm{V})$ intake, barriers to healthy eating, support for healthy eating, and access/use of technology. Eligibility criteria were 1) English-speaking, 2) $40+$ years of age, 3) owned or had access to a smartphone, and 4) self-identify as overweight or obese. Descriptive statistics characterized healthy eating engagement, support for healthy eating, and technology access/use. Chi-squared analyses described the relationship between healthy eating support and likelihood of using health information technology.

Results: Mean age of respondents was 53 years with mean body mass index of 32.2. Only $25 \%$ adhered to CDCrecommended $\mathrm{F} / \mathrm{V}$ consumption guideline, and individuals earning up to $\$ 76,000$ per year were most likely to follow the F/V guideline. Those with a high school education or higher reported adhering to the recommended guideline more so than individuals with less education ( $28 \%$ vs $11 \%)$. More than half $(57 \%)$ of respondents experienced healthy eating barriers, and $52 \%$ reported support from family or friends. Most respondents used their personal computers (92\%) and mobile devices $(82 \%)$ to look up health information. Almost two-thirds (62\%) reported a likelihood of using online health information to support healthy eating engagement. Chi-squared analyses $\left(\chi^{2}\right)$ revealed a significant relationship between healthy eating support and likelihood of using online health information tools, $\chi^{2}(2, \mathrm{n}=60)$ : 7.69, $\left.\mathrm{P}=0.021\right)$.

Conclusion: Working rural women with lower income and less than a high school education require support to eat healthy and are less likely to follow recommended F/V guidelines. Web- and mobile-based technologies can promote healthy behaviors, and our rural sample appears willing to 
engage in digital health care management. Technologists should consider sociodemographic factors in the design/ development of innovations to ensure a tailored intervention for rural women.

\section{P16.03}

\section{A New Midwestern Virtual Data Warehouse From SSM Health and Saint Louis University Health Care Systems}

\author{
Leslie Hinyard, ${ }^{1}$ Jeffrey Scherrer ${ }^{1}$ \\ ${ }^{1}$ Saint Louis University, St. Louis, MO
}

Background: HCSRN membership can lead to transformative growth in research collaboration and funding. Saint Louis University (SLU) has funded the development of a virtual data warehouse (VDW) containing electronic health record data from more than 2 million patients who have received care in SLU's academic medical practice or its partner SSM Health, with the goal of joining the HCSRN and filling the gap of HCSRN members located in the Midwest. Herein, we describe the development of a unique comprehensive VDW encompassing data from two organizationally distinct collaborative entities.

Methods: A merged instance of Epic between SSM Health and SLUCare medical practice provided a unique opportunity for the development of an SSM-SLU VDW. Working collaboratively with both medical organizations, SLU researchers obtained a data use agreement for shared data use across the institutions. SLU researchers consulted with leadership and collaborators within the HCSRN to inform a business plan intended to self-sustain the VDW within 4 years. Upon obtaining institutional support for the project, we recruited an experienced HCSRN VDW developer and established a phase 1 goal to extract a limited data set with monthly updates by June 2020. Phase 2 extends sources of data to new patientreported outcomes, wearables, and geospatial measures.

Results: This unique academic-private health system collaboration began after data use agreement completion. The SSM-SLU VDW will include 23 hospitals, nearly 300 ambulatory care sites, and more than 2 million patients who receive care in Missouri, Illinois, Oklahoma, and Wisconsin. Patient demographics include sufficient samples of poor African American and white as well as urban- and ruralbased patients to offer a unique resource for health disparities research. Publications are expected by the end of 2019, and application to HCSRN membership will be made in 2020 .

Conclusion: The SSM Health-SLU partnership allows for novel health services and outcomes research comparing academic to private sector delivery of care. Bridging two independent organizations with shared data resources presents both challenges and advantages during resource development. Early VDW-user research areas include mental health, infection, asthma, breast cancer, and type 2 diabetes. Investigators have a successful track record of HCSRN collaboration, which should enhance our potential to become members of this national resource.

P16.04

A Web-Based Medication Reconciliation Tool Reduced Medication Discrepancies in a Primary Care Population

Michael Gionfriddo, ${ }^{1}$ Vanessa Duboski, ${ }^{1}$ Melissa Kern, ${ }^{1}$ Bhumika Maddineni, ${ }^{1}$ Yirui Hu, ${ }^{1}$ Eric Wright ${ }^{1}$

${ }^{1}$ Geisinger Health, Danville, $P A$

Background: Having an accurate medication list is a wellrecognized patient safety issue. Unfortunately, patients' medication lists are often inaccurate. We developed a web-based medication reconciliation tool (MedTrue ${ }^{\mathrm{TM}}$ ) to facilitate the collection of accurate medication lists through integration of patient-collected, electronic health record, and administrative claim medication information and a display that integrates within the electronic health record. Effectiveness of this tool in improving the accuracy of medication lists is not known.

Methods: We conducted a cluster randomized trial within 6 primary care clinics within an integrated health delivery system. Sites were selected in collaboration with clinic leadership and randomized in a 1:1 fashion to intervention (MedTrue) or usual care using a random number generator. Patients were eligible for inclusion if they were seen at a participating clinic for a visit with their primary care provider, if they were 18 years of age or older, if they spoke English, and if, in the intervention arm, both the patient and a health care provider were exposed to MedTrue. After an eligible visit, patients were approached by a trained pharmacist, obtained consent, and performed a best possible medication history. Our primary outcome was the rate of medication discrepancies, defined as a composite rate of medication additions, removals, or modifications (change in dose, frequency, etc). Continuous outcomes are reported using mean and standard deviation, and a zero-inflated Poisson regression model was used to evaluate the impact of MedTrue.

Results: Overall, 224 patients were recruited and underwent a best possible medication history with the pharmacist (118 usual care, 106 MedTrue). The most common discrepancies identified were modifications, with a mean of 2.68 modifications identified per patient in usual care and 1.54 modifications per patient identified among those who used the tool. For the primary composite outcome, after adjusting for age, sex, and Charlson comorbidity index, patients in MedTrue had a lower rate of medication discrepancies compared to usual care (rate ratio: $0.84,95 \%$ CI: $0.74-0.94$; $\mathrm{P}=0.0025$ ).

Conclusion: MedTrue reduced the rate of medication list discrepancies among a population of patients seen in primary care. Future studies should assess how best to implement the tool in primary care practices. 


\section{P16.05}

The Dreem Headband as an Alternative to Polysomnography for Electroencephalogram Signal Acquisition and Sleep Staging

Pierrick Arnal, ${ }^{1}$ Valetin Thorey, ${ }^{1}$ Michael Ballard, ${ }^{1}$ Albert Bou Hernandez, ${ }^{1}$ Antoine Guillot, ${ }^{1}$ Hugo Jourde, ${ }^{2}$ Mason Harris, ${ }^{1}$ Mathias Guillard, ${ }^{2}$ Pascal Van Beers, ${ }^{2}$ Mounir Chennaoui, ${ }^{2}$ Fabien Sauvet ${ }^{2}$

${ }^{1}$ Dreem, New York, NY; ${ }^{2}$ Fatigue and Vigilance Unit, French Armed Forces Biomedical Research Institute (IRBA), Paris, France

Background: Despite the central role of sleep in our lives and the high prevalence of sleep disorders, sleep is still poorly understood. The development of ambulatory technologies capable of monitoring brain activity during sleep longitudinally is critical to advancing sleep science and facilitating the diagnosis of sleep disorders. We introduced the Dreem headband (DH) as an affordable, comfortable, and user-friendly alternative to polysomnography (PSG). The purpose of this study was to assess the signal acquisition of the $\mathrm{DH}$ and the performance of its embedded automatic sleep-staging algorithms compared to the gold-standard clinical PSG scored by 5 sleep experts.

Methods: 31 subjects completed an overnight sleep study at a sleep center while wearing both a PSG and the DH simultaneously. We assessed 1) the electroencephalogram (EEG) signal quality between the DH and the PSG; 2) the heart rate, breathing frequency, and respiration rate variability (RRV) agreement between the $\mathrm{DH}$ and the PSG; and 3) the performance of the DH's automatic sleep staging according to American Academy of Sleep Medicine guidelines vs PSG sleep experts' manual scoring.

Results: Results demonstrate a strong correlation between the EEG signals acquired by the DH and those from the PSG, and the signals acquired by the DH enable monitoring of alpha (r: $0.71 \pm 0.13$ ), beta (r: $0.71 \pm$ $0.18)$, delta (r: $0.76 \pm 0.14$ ), and theta (r: $0.61 \pm 0.12$ ) frequencies during sleep. Mean absolute error for heart rate, breathing frequency, and RRV was $1.2 \pm 0.5 \mathrm{bpm}$, $0.3 \pm 0.2 \mathrm{cpm}$, and $3.2 \% \pm 0.6 \%$, respectively. Automatic sleep staging reached an overall accuracy of $83.5 \% \pm$ $6.4 \%$ (F1 score : $83.8 \pm 6.3$ ) for the $\mathrm{DH}$, compared with an average of $86.4 \% \pm 8.0 \%$ (F1 score: $86.3 \pm 7.4$ ) for the 5 sleep experts.

Conclusion: These results demonstrate the capacity of the DH to both precisely monitor sleep-related physiological signals and process them accurately into sleep stages. This device paves the way for high-quality, large-scale, longitudinal sleep studies.
P16.06

Mixed-Methods Evaluation of Parents' Unmet Needs and Experiences Regarding Behavioral Intervention Technologies for Common Behavioral Health Problems

Sean O'Dell, ${ }^{1}$ Heidi Fisher, ${ }^{1}$ Victoria Schlieder, ${ }^{1}$ Tracey Klinger, ${ }^{1}$ Rachel Kininger, ${ }^{1}$ McKenna Corlis, ${ }^{1}$ Amanda Ferriola $^{1}$

\section{${ }^{1}$ Geisinger Health, Danville, PA}

Background: Behavioral intervention technologies (BITs) are an emerging modality for electronic behavioral innovations across a wide range of behavioral, emotional, and cognitive outcomes. However, few BITs have been tested with parents in real-world settings. We conducted a mixed-methods evaluation of unmet needs to inform subsequent iterative user testing.

Methods: An exploratory sequential design was used, in which focus group interviews were conducted with primary care and behavioral health stakeholders. Then, analysis of these data was used to inform a parent survey that included 17 selected behavioral health topics corresponding to the American Academy of Pediatrics' Bright Futures modules.

Results: Qualitative results are collected, transcribed, coded, and will be further analyzed using Atlas.ti. Survey results were implemented using Qualtrics, have been cleaned, and await analysis once qualitative results are finalized (anticipated December 2019). Preliminary results show several preferred BIT domains addressing both current and future concerns for their children.

Conclusion: We found unique advantages to this mixedmethods approach that will inform design and evaluation functions as the project enters the design, evaluation, and sustainability phases. Implementation and partnership challenges will be discussed, as will systems-level factors that support and constrain design for dissemination in our setting.

\section{MENTAL HEALTH AND SUBSTANCE USE DISORDERS/ADDICTION}

OA4.01

Accuracy of an Opioid Withdrawal Risk Assessment Screening Tool for Hospital Patients

Kimberly Williams, ${ }^{1}$ Claudine Jurkovitz, ${ }^{1}$ Richard Caplan, ${ }^{1}$ Beverly Wilson, ${ }^{1}$ Alexa Meinhardt, ${ }^{1}$ Bayo Gbadebo, ${ }^{1}$ Terry Horton ${ }^{1}$

\section{${ }^{1}$ ChristianaCare, Wilmington, $D E$}

Background: Opioid use disorder and withdrawal may go undetected among patients admitted for general medical reasons, raising the risk of withdrawal during hospitalization. 
To address this, we implemented a standardized method to screen and treat opioid withdrawal in the hospital, including the development of an Opioid Withdrawal Risk Assessment (OWRA) tool for universal screening. This study aimed to evaluate the accuracy of the OWRA in identifying patients at risk for opioid withdrawal.

Methods: We evaluated adult patients who were administered the OWRA from 2016 to 2017. A retrospective analysis compared OWRA results to the Clinical Opiate Withdrawal Scale (COWS), the gold standard used to assess opioid withdrawal symptoms and evaluate withdrawal severity. Admitted patients were asked: 1) Have you used heroin or prescription pain medicines other than prescribed in the last week? and 2) Do you get sick if you can't use heroin, methadone, or prescription pain medications? Patients who answered "Yes" to either OWRA question were considered a positive screen and administered COWS. A positive COWS (score of $\geq 8$ ) triggered evaluation and treatment for opioid withdrawal. A sample of patients who screened negative on the OWRA (ie, "No" to both questions) were administered COWS to evaluate the OWRA's accuracy. We used a Bayesian approach to estimate the prevalence of opioid withdrawal as well as OWRA's sensitivity, specificity, positive predictive value (PPV), and negative predictive value (NPV).

Results: Of 22,441 patients, 21,935 (97.7\%) screened negative on the OWRA and $506(2.3 \%)$ screened positive. Of patients with a positive OWRA, $28.1 \%$ had a positive COWS. We administered COWS to a sample of 646 patients with a negative OWRA. Of the 646 OWRAnegative patients, $2(0.31 \%)$ had a positive COWS. The estimated opioid withdrawal prevalence was $1.15 \%$ (standard deviation: $0.26 \%$ ). The OWRA' s sensitivity was 57.3\% (95\% CI: 35.6\%-81.1\%), specificity 98.4\% (95\% CI: 98.3\%-98.4\%), PPV 31.7\% (95\% CI: 27.8\%-35.7\%), and NPV 99.5\% (95\% CI: 98.8\%-99.9\%).

Conclusion: Given its high specificity and NPV, the OWRA tool may effectively exclude patients not at risk for opioid withdrawal, successfully limiting the instance of false-positive assessments and ensuring that treatment is administered appropriately. Additional research is needed to further assess factors that may affect the OWRA's sensitivity and PPV.

\section{OA4.02}

Instrumental Variable Analysis of Lethal Means Assessment in Patients Reporting Suicide Ideation and Subsequent Risk for Suicide Outcomes

Jennifer M. Boggs, ${ }^{1}$ Arne Beck, ${ }^{1}$ Debra P. Ritzwoller, ${ }^{1}$ Catherine Battaglia, ${ }^{2}$ Heather D. Anderson, ${ }^{2}$ Richard C. Lindrooth $^{2}$

\section{${ }^{1}$ Kaiser Permanente Colorado, Denver, CO; ${ }^{2}$ University of Colorado, Aurora, CO}

Background: Counseling on access to lethal means (ie, firearms, medications) is highly recommended for patients with suicide risk, but there are no formal evaluations of its impact in real-world settings. We evaluated the impact of lethal means assessment delivered during medical and mental health visits on suicide outcomes. Randomized trials would not be ethical to answer this question, and there is high likelihood for confounding with traditional regression adjustment. We implemented a cohort design using an instrumental variable to address this confounding. Methods: The sample included adult patients of Kaiser Permanente Colorado who endorsed suicide ideation on the Personal Health Questionnaire-9 (PHQ-9) depression screener during medical and mental health visits from 2010 to 2016. Provider documentation of lethal means assessment in clinical notes was collected using a validated natural language processing program. The instrument was the practice-level rate of lethal means assessment, which had substantial variation by location, department, and provider specialty visited. The instrument helped adjust for unmeasured patient characteristics from clinical interviews that influence provider decisions to ask about lethal means. The main outcomes included any ICD-9 or ICD-10 code for self-inflicted injury/death within 180 days of index PHQ-9. Demographic, diagnostic, and treatment co-variates were included in a 2-stage residual inclusion model to evaluate the impact of lethal means assessment on suicide outcomes.

Results: We found 33\% of patients with suicide ideation reported on the PHQ-9 were exposed to lethal means assessment in the 30 days following risk identification. Lethal means assessment reduced the risk of a suicide attempt or death within 180 days from $3.3 \%$ to $0.83 \%$, with a relative risk of 0.25 ( $\mathrm{P}=0.034,95 \% \mathrm{CI}$ : $0.069-0.9)$.

Conclusion: The reduction in suicide risk that we detected may be due to lethal means assessment as well as unmeasured suicide prevention strategies that may co-occur with it. Instrumental variable approaches are an important and underutilized tool for detecting effects with research questions that are unethical to pose with randomized trials. Mental health providers should expand the use of counseling on access to lethal means, along with co-occurring safety planning practices, to all patients who report suicide ideation.

\section{OA4.03 \\ Measuring Quantitative Traits of Alcohol Use Disorder Using the Alcohol Use Disorders Identification Test}

Patrick Asdell, ${ }^{1}$ Donielle Beiler, ${ }^{1}$ Stephanie Ranck, ${ }^{1}$ Vanessa Troiani ${ }^{1}$

${ }^{1}$ Geisinger Health, Danville, PA

Background: Alcohol use disorder (AUD) is a common, devastating substance use disorder (SUD), with typical onset in adolescence or young adulthood and a chronic 
relapsing course of illness, resulting in disability, billions of dollars lost in economic activity, and greater than 3-fold increased mortality. It is estimated that persons of European ancestry have a lifetime prevalence of severe AUD of $15.8 \%$, although AUD is underdiagnosed in most health systems. Herein, we assess the utility of using patient portal messaging to obtain alcohol use information from patients.

Methods: We report data from 680 patients who enrolled in our study and completed the Alcohol Use Disorders Identification Test (AUDIT) via a patient messaging portal at Geisinger. Education and monitoring processes were implemented for patients who 1) scored above threshold on the AUDIT, indicating probable AUD, and 2) requested that their AUDIT score be sent to their medical provider. We also examine patients' willingness to share the scores of this screener with their physician, patient awareness of alcohol treatment options within the health system, and the impact of online learning materials regarding excessive alcohol use. Recruitment is ongoing.

Results: Of patients that use alcohol, 25\% were at some risk of AUD based on AUDIT scores. A majority of patients (59\%) requested that their AUDIT scores be forwarded to their physicians, while a substantial portion (36\%) were unaware that medication-assisted treatment is an option for AUD. Outcome monitoring is ongoing but presently indicates that multiple individuals have been referred to social work, medication-assisted treatment, and inpatient substance use disorder treatment. Other patients have reported reduction in alcohol consumption since completing the survey and/or have had a discussion with their primary care physician about alcohol use, following enrollment in this study.

Conclusion: These results suggest that use of patient portal messaging for substance use disorder screeners may be a productive method that fosters communication between patients and providers, stratifies patients based on the level of treatment needed, and records quantitative substance use disorder data on individual patients using a mechanism amenable to scaling for high throughput data collection.

\section{OA4.04 \\ Long-Term Effectiveness of Online Mindfulness- Based Cognitive Therapy for Reducing Residual Depressive Symptoms: A Randomized Pragmatic Trial}

Arne Beck, ${ }^{1}$ Zindel Segal, ${ }^{2}$ Sona Dimidjian, ${ }^{3}$ Jennifer M. Boggs, ${ }^{1}$ Rachel Vanderkruik, ${ }^{3}$ Christina Metcalf, ${ }^{3}$ Robert Gallop, ${ }^{4}$ Jennifer Felder, ${ }^{5}$ Joseph Levy ${ }^{3}$

${ }^{1}$ Kaiser Permanente Colorado, Denver, CO; ${ }^{2}$ University of Toronto, Toronto, Canada; ${ }^{3}$ University of Colorado Boulder, Boulder, CO; ${ }^{4}$ West Chester University, West Chester, PA; ${ }^{5}$ University of California, San Francisco, San Francisco, CA
Background: Patients with residual depressive symptoms experience functional impairments and increased risk for recurrence of major depressive disorder (MDD). They also face a "care gap," as few resources are available to manage the lingering effects of their illness. The objective of this study was to evaluate the effectiveness for treating residual depressive symptoms with Mindful Mood Balance (MMB), a web-based application that delivers mindfulness-based cognitive therapy.

Methods: A 2-group randomized pragmatic trial was conducted at Kaiser Permanente Colorado, recruiting adults with residual depressive symptoms (ie, Personal Health Questionnaire-9 [PHQ-9] scores between 5 and 9) and 1 or more prior episodes of major depressive disorder. Participants were recruited from behavioral health and primary care clinics between March 2015 and November 2018. Both groups received usual depression care (UDC), and the MMB group also received 8 sessions delivered online over 3 months plus minimal phone/email coaching support. Primary outcomes were reduction in PHQ-9 scores, rates of depressive relapse (PHQ-9 of $>15$ ), and rates of remission (PHQ-9 $<5$ ). Secondary outcomes included depression free days (DFD), anxiety symptoms (per the 7-item Generalized Anxiety Disorder [GAD-7] scale), and functional status (per the SF-12 patient questionnaire). Outcomes were assessed over a 15-month period, comprising a 3-month intervention interval and a 12-month follow-up.

Results: Among 460 randomized participants (230 per group), data were analyzed for the intent to treat sample. Participants had a mean age of 48 years, and $76 \%$ were women. Those who received MMB+UDC had significantly greater mean reductions in residual depressive symptoms than those receiving UDC alone $(\mathrm{P}<0.02)$. A significantly greater proportion of patients achieved remission in MMB+UDC relative to UDC $(\mathrm{P}<0.01)$, and rates of depressive relapse also were significantly lower in $\mathrm{MMB}+\mathrm{UDC}$ relative to UDC (hazard ratio: $0.61,95 \% \mathrm{CI}: 0.39-0.95$, $\mathrm{P}<0.03)$. Secondary outcomes indicated an additional 34 depression-free days for $\mathrm{MMB}+\mathrm{UDC}$ relative to UDC $(\mathrm{P}<0.02)$, mean decreases in anxiety $(\mathrm{P}<0.004)$, and improved mental functioning $(\mathrm{P}<0.0003)$, but not physical functioning.

Conclusion: In the largest online treatment trial of residual depressive symptoms, MMB resulted in significant improvement in depression and functional outcomes compared to routine depression care. MMB may offer a scalable approach for the management of residual depressive symptoms. 
P1.01

Agent-Specific Analysis of Opioid Distribution Trends in U.S. Hospitals From 2000 to 2018

Sarah Eidbo, ${ }^{1}$ Varkey Mathew, ${ }^{1}$ Daniel Kaufman, ${ }^{1}$ Stephanie Nichols, ${ }^{2}$ Kenneth McCall, ${ }^{2}$ Brian Piper ${ }^{1}$

${ }^{1}$ Geisinger Health, Danville, PA; ${ }^{2}$ Department of Pharmacy Practice, University of New England, Portland, ME

Background: The United States is currently enduring a devastating opioid crisis. More than 130 people die every day from opioid overdoses, and in 2017 alone, 47,000 Americans died from an opioid overdose. The U.S. Department of Health and Human Services estimates that $40 \%$ of opioid overdose deaths involve a prescription opioid. The purpose of this project was to analyze trends in use and distribution of opioid compounds by hospitals in the United States from 2000 to 2018. The Automation of Reports and Consolidated Orders System (ARCOS) is a drug reporting system for substances controlled for by the Drug Enforcement Administration, which reports on an array of opioids and opioid derivatives. Opioids monitored included buprenorphine, codeine, fentanyl, hydrocodone, hydromorphone, meperidine, methadone, morphine, oxycodone, oxymorphone, powdered opium, remifentanil, and tapentadol.

Methods: Data from ARCOS Report 5 and Report 7 from 2000 to 2018 were utilized for an observational study on hospital opioid distribution in the United States, excluding U.S. territories. The programs GraphPad Prism and Excel were used to graph total weights of the studied opioids. The morphine milligram equivalent (MME) was calculated using oral conversion factors for each opioid. A paired $t$-test was run on the MME per person per state to compare 2012, the year with the highest U.S. MME total, and 2018, the year of the most recent ARCOS data release.

Results: Hospital opioid use in the United States has seen a statistically significant $31.1 \%$ net decrease from 2012 to 2017. When totals were calculated correcting for the opioids no longer reported on in 2018, a statistically significant decrease of $42.24 \%$ was seen between 2009 and 2018. Codeine and meperidine have seen overall decreases in hospital use from 2000 to 2018. Buprenorphine has seen overall increases in hospital use from 2000 to 2018. Tapentadol has been declining in use since 2017 . Oxycodone, hydromorphone, hydrocodone, methadone, morphine, powdered opium, oxymorphone, and fentanyl are experiencing a slow decline in hospital use.

Conclusion: Despite the opioid crisis continuing to ravage communities throughout the United States, hospitals are steadily decreasing their overall opioid use.
P1.02

A Randomized Controlled Pilot of a Clinical Decision Support Tool to Improve Primary Care of Opioid Use Disorder

Rebecca Rossom, ${ }^{1}$ JoAnn Sperl-Hillen, ${ }^{1}$ Patrick J. O'Connor, ${ }^{1}$ A. Lauren Crain, ${ }^{1}$ Laurel Nightingale, ${ }^{1}$ Anne Pylkas, ${ }^{1}$ Kristen Huntley, ${ }^{2}$ Gavin Bart ${ }^{3}$

${ }^{1}$ HealthPartners, Bloomington, MN; ${ }^{2}$ National Institutes of Health, Bethesda, MD; ${ }^{3}$ University of Minnesota, Minneapolis, MN

Background: Despite an opioid epidemic, most Americans with opioid use disorder (OUD) do not receive medical care for their OUD. A clinical decision support (CDS) tool designed for primary care providers (PCP) could improve this treatment gap. The primary objective of this study was to build and pilot test an OUD-CDS tool with PCPs. Secondary objectives were to achieve high use rates, improve PCP confidence in assessing and treating OUD, and achieve high PCP approval rates.

Methods: Design: 55 PCPs in a Midwestern health care system participated. PCPs waivered to prescribe buprenorphine $(\mathrm{n}=8)$ were assigned to the intervention. Nonwaivered PCPs $(n=47)$ were randomized to the intervention $(\mathrm{n}=24)$ or control $(\mathrm{n}=23)$ group. Intervention: Intervention PCPs received access to the OUD-CDS alerting them to high-risk patients. The OUD-CDS included modules for screening and diagnosis, treatment selection, treatment guidance, and other recommended care. Control PCPs provided care as usual.

Results: Testing in the electronic health record using simulated and real patients determined the OUD-CDS was functional and accurate. PCPs used the OUD-CDS in 5\% of targeted encounters, less than the goal of $60 \%$. Intervention PCPs reported increased confidence in screening for OUD following the pilot, whereas control PCPs did not. Confidence in diagnosing OUD increased in nonwaivered intervention PCPs but not in waivered intervention or control PCPs. Most intervention PCPs would recommend the OUD-CDS to a colleague and found it helpful with screening for OUD, discussing and prescribing medications for OUD, and prescribing naloxone.

Conclusion: The OUD-CDS improved PCP confidence in screening and diagnosing OUD despite low use rates. Qualitative data are guiding development of modifications to increase CDS use rates. 
P1.03

Validation of a DSM-5 Opioid Use Disorder Severity Score Derived From Health Record Review

Anthony Seiwell, ${ }^{1}$ Sarah Palumbo, ${ }^{2}$ Alexia Perrin, ${ }^{1}$ Tabitha Milliken, ${ }^{1}$ Ashlee Olenginski, ${ }^{1}$ Samantha Hoffman, ${ }^{1} \quad$ Kayleigh Adamson, ${ }^{1}$ Sarathbabu Krishnamurthy, ${ }^{1}$ Raghu Metpally, ${ }^{1}$ Kortney McBryan, ${ }^{1}$ Stephanie Ranck, ${ }^{1}$ Richard Crist, ${ }^{3}$ Glenn Doyle, ${ }^{3}$ Thomas Ferraro, ${ }^{3}$ Wade Berrettini, ${ }^{3}$ Janet Robishaw, ${ }^{2}$ Vanessa Troiani ${ }^{1}$

${ }^{1}$ Geisinger Health System, Danville, PA; ${ }^{2}$ Florida Atlantic University, Boca Raton, FL; ${ }^{3}$ University of Pennsylvania, Philadelphia, PA

Background: Opioid use disorder (OUD) is ideally diagnosed based on a clinician interview that determines severity based on DSM-5 criteria, resulting in a severity score. However, many patients are prescribed opioids without undergoing such an interview, and thus may not be diagnosed with OUD, despite meeting criteria. Thus, it would be advantageous to have a quantitative scoring algorithm that can determine a DSM-5 OUD severity score based on existing health record data.

Methods: We conducted in-depth chart review of 600 patients; 400 patients were used to develop a proxy DSM5 risk score to predict severity of OUD (200 at high risk of OUD and 200 at low risk). We then validated this proxy DSM-5 score in 200 additional patients who have confirmation of OUD diagnostic status and a self-reported questionnaire that assesses DSM-5 criteria for OUD.

Results: We used our proxy DSM-5 scoring criteria to predict OUD severity among opioid-using patients. In the high-risk patients, there was a high prevalence of moderate to severe OUD, with 94 patients (47\%) meeting the criteria for severe OUD and 50 patients (25\%) meeting the criteria for moderate OUD. Validation chart review of an independent sample of patients with confirmed diagnosis is ongoing.

Conclusion: These results indicate that many DSM-5 criteria for OUD can be determined via in-depth chart review. Following a validation review on an independent sample of patients with confirmed OUD and quantitative scores for DSM-5 OUD criteria, we will determine whether the same criteria can be extracted via automated methods using discrete electronic health record variables.

\section{$\mathrm{P} 1.04$}

Degree of Bystander-Patient Relationship and Prehospital Care for Opioid Overdose

Molly McCann, ${ }^{1}$ Todd Jusko, ${ }^{2}$ Courtney Jones, ${ }^{2}$ Christopher Seplaki, ${ }^{2}$ Jeremy Cushman ${ }^{2}$

${ }^{1}$ Henry Ford Health System, Detroit, Ml; ${ }^{2}$ University of Rochester Medical Center, Rochester, NY
Background: Across the spectrum of patient care for opioid overdose, an important yet frequently overlooked feature is the bystander. For other acute medical events, such as cardiac arrest and stroke, research supports that the presence of a bystander is associated with better outcomes. Despite the similarities, however, this well-established conceptual framework has yet to be applied in the context of overdose. Our objective was to assess whether the degree of bystander-patient relationship is associated with prehospital care for the treatment of opioid overdose.

Methods: A retrospective cohort study was conducted among adults who received naloxone administration in the prehospital setting for suspected opioid overdose. Patients were identified using a preexisting longitudinal registry documenting all prehospital administrations of naloxone by first responders in a midsized community in New York. Individuals who received at least 1 administration for a suspected opioid overdose from June 1,2016 , to July 31 , 2018, with available emergency medical services and medical record data were eligible for study inclusion. Bystander type was defined as Close (spouse/family), Proximal (friends), and Distal (no relation to patient). The association between bystander type and prehospital patient care outcomes were estimated using logistic and linear regression models.

Results: A total of 610 opioid overdose encounters, equating to 545 unique patients, were identified from the registry. The sample makeup was predominately male (67.2\%), white $(73.6 \%)$, and $25-34$ years of age $(34.9 \%)$. Among patients who had Proximal-related bystanders during their encounter, the time to naloxone administration was decreased by 2.40 minutes, compared to Distal-related, after adjusting for covariates (95\% CI: -4.6 to -0.2$)$. Overdose encounters with 911 dispatch codes more indicative of opioid overdose (ie, "overdose/poisoning" vs "unconscious/fainting") were associated with having a Close/Proximal bystander present relative to a Distal bystander (odds ratio: $1.9,95 \%$ CI: 1.1 to 3.4 for Close; odds ratio: 3.7, 95\% CI: 1.9 to 7.3 for Proximal). Conclusion: We found that having a Proximal bystander during an overdose event is associated with dispatch codes indicative of an overdose and shorter time to naloxone administration. These findings offer an alternative focal point for interventions aimed at spreading overdose awareness and harm reduction strategies.

\section{P1.05 \\ Degree of Bystander-Patient Relationship and Its Association With Repeated Opioid Overdose Events}

Molly McCann, ${ }^{1}$ Todd Jusko, ${ }^{2}$ Courtney Jones, ${ }^{2}$
Christopher Seplaki, ${ }^{2}$ Jeremy Cushman ${ }^{2}$

${ }^{1}$ Henry Ford Health System, Detroit, MI; ${ }^{2}$ University of Rochester, Rochester, NY

Background: Across the spectrum of patient care for opioid overdose, an important yet frequently overlooked feature is the bystander. For other acute medical events, such as cardiac 
arrest and stroke, research supports that bystander presence is associated with better outcomes. Despite the similarities, this well-established conceptual framework has yet to be applied in the context of overdose. Our objective was to assess whether the degree of bystander-patient relationship is associated with repeated opioid overdose events.

Methods: A retrospective cohort study was conducted among adults who received naloxone administration in the prehospital setting for suspected opioid overdose. Patients were identified using a preexisting longitudinal registry documenting all prehospital administrations of naloxone by first responders in a midsized community in New York. Individuals who received at least 1 administration for a suspected opioid overdose from June 1, 2016, to July 31, 2018, with available emergency medical services and medical record data were eligible for study inclusion. Bystander type was defined as Close (spouse/family), Proximal (friends), and Distal (no relation to patient). The association between bystander type and repeated overdose event was estimated using logistic regression models.

Results: A total of 610 opioid overdose encounters, among 545 unique patients, were identified from the registry. The sample makeup was predominately male $(67.2 \%)$, white (73.6\%) and 25-34 years of age (34.9\%). More than $11 \%$ of patients experienced a repeated overdose event within the study time frame, and 5.6\% experienced at least 1 within 90 days of their initial overdose encounter. Having a Proximal bystander present during their initial encounter was associated with $36 \%$ lower risk of a repeated overdose event within 90 days, compared to those with Distal bystanders (odds ratio: $0.6,95 \% \mathrm{CI}$ : 0.2 to 1.9 ). Conversely, risk of repeating was $53 \%$ higher for individuals with Close bystanders, relative to Distal, but again this difference did not reach statistical significance (95\% CI: 0.7 to 3.4 ).

Conclusion: Although we did not find a significant association between bystander type and odds of repeated overdose event, these results offer an opportunity to further evaluate the role of the bystander in the context of one's prospective substance use disorder recovery.

\section{P1.06}

Exploring the Mechanisms of Substance Use Disorders Among Veterans: A Team Science Approach

\section{Margaret Kucia, ${ }^{1}$ Heather Basehore ${ }^{1}$}

\section{${ }^{1}$ Coatesville VA Medical Center, Coatesville, PA}

Background: Veterans face significant health disparities as compared to nonveteran counterparts, and the complex, multifaceted medical and social determinants underlying these health disparities present a growing public health problem. Most notably, veterans suffer from traumatic brain injury (TBI) at rates disproportionate with nonveterans, and this study aims to better characterize the nature of TBI sequelae and identify a causal directionality between TBI and subsequent development of substance use disorders through an interdisciplinary research team.

Methods: Data was gathered through patient interviews conducted with validated Structured Clinical Interview for DSM-Research Version (SCID-RV), PTSD Checklist for DSM-5 (PCL-5), Patient-Reported Outcomes Measurement Information System (PROMIS-29), and Ohio State University TBI questionnaires to assess psychological comorbidities, history of lifetime TBI, posttraumatic stress disorder (PTSD), activity limitations, demographics, social factors, and reported medical and psychological history. Measures of impulsivity were assessed through computer-based testing. Additional data mining was conducted through electronic health records to identify periods of service, disability categorizations, combat exposure, and disability percentages. Principal component analysis, visualizations, and statistical analyses were used to identify clustering, risk stratification, and predictive capacity among patient comorbidities and risk factors.

Results: This study represents one aim of a multifaceted investigation centered on identifying mechanisms and treatment strategies to counter addiction susceptibility after TBI across all populations, with an eventual goal of developing medical intervention to attenuate TBI pathology and effects on addiction. Preliminary results aim to identify entry points for intervention and development of risk assessment tools to assist veteran and health care teams across the care continuum. Conclusion: This broad-based exploration supports continued research in mitigating the sequelae of TBI and reducing health disparities in veterans and underscored the value of interdisciplinary team problem-solving. Most notably, the use of dimension reduction and comparison across clinical, psychological, demographic, and social data elements enabled identification of avenues for clinical and psychosocial intervention, risk assessment, and preventive methods.

\section{P12.01}

The Development, Optimization, and Pilot Testing of a Brief Psychological Intervention for Chronic Pain in Primary Care

Lisa Miller-Matero, ${ }^{1}$ Mary Kate Miller, ${ }^{1}$ Leah Hecht, ${ }^{1}$ Bethany Pester, ${ }^{1}$ Kirsti Autio, ${ }^{1}$ Brian Ahmedani ${ }^{1}$

\section{${ }^{1}$ Henry Ford Health System, Detroit, MI}

Background: Approximately $25 \%$ of primary care patients have a chronic pain condition, half of which also have a psychiatric diagnosis. Although patients with chronic pain can benefit from behavioral interventions, few patients who are referred by their primary care provider (PCP) to see a behavioral health specialist at an outside clinic follow through with the referral. Thus, offering evidence-based behavioral treatments in primary care may improve patient engagement and outcomes. The purpose of this presentation is to discuss the development, optimization, and pilot testing 
of a brief, psychological intervention for chronic pain in a primary care clinic.

Methods: A treatment manual was developed by a clinical psychologist to integrate a brief, psychological intervention in a primary care clinic. The intervention was optimized after conducting focus groups with 9 psychologists, 9 PCPs, and 9 patients. The manual was further refined after an open trial with 5 participants with chronic pain. Currently, a randomized controlled trial of the 5-session intervention is being conducted in a primary care clinic $(\mathrm{N}=60)$. The intervention integrates components from evidence-based interventions for chronic pain, including psychoeducation, cognitive behavioral therapy, mindfulness, and acceptance. Participants complete a baseline assessment and a postintervention assessment.

Results: The changes made to the treatment manual and protocol based on feedback from the focus groups and open trial will be presented. To date, 34 participants have been randomized. More than half of participants in the intervention group completed all 5 sessions (64.3\%). Most participants completed the postintervention assessment (92.9\%). On a 1-5 point scale, patients liked the intervention (mean: 4.45 , standard deviation [SD]: 0.52), found the content to be useful (mean: 4.91, SD: 0.30), and thought it was helpful (mean: 4.64, SD: 0.67).

Conclusion: Overall, it appears feasible to offer a brief, psychological intervention for chronic pain in primary care. Barriers to attending treatment will be discussed as well as protocol changes being implemented. The information gathered in this pilot trial will be used to inform a fully powered, multisite randomized controlled trial leveraging the HCSRN to evaluate efficacy of the integrated intervention on physical functioning, psychological distress, mindfulness, and acceptance of pain.

P12.02

Measurement of Autonomic Dysregulation in Pediatric Patients With Autism Using Health Record Data

Antoinette DiCriscio, ${ }^{1}$ Donielle Beiler, ${ }^{1}$ Patrick Asdell, ${ }^{1}$ Jaclyn Smith, ${ }^{1}$ Shannon Dickey, ${ }^{1}$ Vanessa Troiani ${ }^{1}$

\section{${ }^{1}$ Geisinger Health, Danville, PA}

Background: Autism spectrum disorder (ASD) is characterized by social communication impairments and repetitive behaviors. Sleep disturbances, gastrointestinal problems, and atypical heart rate also are commonly observed in patients with ASD and may all relate to an underlying dysfunction of the autonomic nervous system (ANS). ANS dysfunction has not been well-characterized in patients with ASD, as it is not part of the core diagnostic criteria. The overall objective of the current study was to quantitatively characterize individual differences in ANS function that may scale with the presence and severity of ASD symptoms.

Methods: We assessed ANS dysfunction in children with and without ASD using two methods: 1) a standardized clinical research questionnaire of autonomic symptoms in pediatric patients (Pediatric Autonomic Symptoms Scale [PASS]); and 2) a chart review of patients' electronic health records (EHR) that coded for the presence and/or absence of the behaviors measured by the PASS. This procedure was completed in 3 groups of patients, including those with ASD diagnoses with no known genetic cause $(n=159)$, patients with a de novo copy number variation (CNV) that confers risk for the development of ASD $(n=244)$, and age- and sex matched controls $(n=213)$. Symptoms of ANS dysregulation were assessed across 4 main categories: 1) mood, behavior, and emotion; 2) secretomotor, sensory integration; 3) urinary, gastrointestinal, and digestion; and 4) circulation, thermoregulation, circadian function, and sleep/wake cycles. Results: Results from the chart review indicate an increased rate of ANS symptoms, specifically those related to atypical secretomotor activity, gastrointestinal function, heart rate, and sleep-related problems in patients with ASD and/or a de novo copy number variation relative to controls. Data collection of the parental report of the PASS is ongoing, and future planned analyses will compare ANS symptoms derived from EHR review to parental report of symptoms. We will also investigate the degree to which indices of ANS dysfunction predict clinical features of ASD.

Conclusion: These results indicate that EHR review is a potentially useful method for quantifying variance in ANS dysfunction. Further, we show that symptoms of ANS dysfunction are more prevalent in children with ASD and genetic disorders conferring risk for ASD.

P12.03

Antidepressant Use and Suicide Mortality

Hsueh-Han Yeh, ${ }^{1}$ Joslyn Westphal, ${ }^{1}$ Frances Lynch, ${ }^{2}$ Rebecca Rossom, ${ }^{3}$ Beth Waitzfelder, ${ }^{4}$ Gregory Simon, ${ }^{5}$ Christine Y. Lu, ${ }^{6}$ Arne Beck, ${ }^{7}$ Stephen Waring, ${ }^{8}$ Ashli Owen-Smith, ${ }^{9}$ Jennifer M. Boggs, ${ }^{7}$ Brian Ahmedani ${ }^{1}$

${ }^{1}$ Henry Ford Health System, Detroit, MI; ${ }^{2}$ Kaiser Permanente Northwest, Portland, OR; ${ }^{3}$ HealthPartners, Bloomington, MN; ${ }^{4}$ Kaiser Permanente Hawaii, Honolulu, HI; ${ }^{5}$ Kaiser Permanente Washington, Seattle, WA; ${ }^{6}$ Harvard Pilgrim Health Care, Boston, MA; ${ }^{7}$ Kaiser Permanente Colorado, Denver, CO; ${ }^{8}$ Essentia Health, Duluth, MN; ${ }^{9}$ Kaiser Permanente Georgia, Atlanta, GA

Background: Depression is one of the most common underlying mental health disorders related to suicide death. The use of antidepressants has raised concerns of increased suicidal thoughts or behavior. Previous studies have shown mixed results regarding the association between antidepressant use and suicide ideation or attempts, and there are a lack of studies examining the relationship between antidepressant use and suicide death. This study aims to fill this gap.

Methods: Data were obtained from 8 health care systems in 
the Mental Health Research Network. This study used a casecontrol design. Cases were identified as those who died by suicide between 2000 and 2015 and had a clinical diagnosis of depression in the year before the index date. To minimize potential bias introduced by poor access to antidepressants and depression severity while comparing medication adherence, a total of 652 cases were included in the analysis who had at least 1 prescription $0-6$ months and at least 1 prescription 7-12 months prior to the index date. Eligible controls were those who had depression diagnoses and met the same criteria of antidepressant exposure. Each case was matched with 5 controls by age, sex, and enrollment period. A cumulative medication adherence (CMA) metric was inclusive of medication switches to different antidepressants and calculated as the proportion of days that the medication was covered. $T$-tests were used to test the mean difference of CMA between cases and controls. Conditional logistic regression was used to investigate the relationship between CMA and suicide mortality.

Results: Patients who died by suicide had an average of $75.4 \%$ of days covered by antidepressants compared to $78.7 \%$ of days covered for the patients in the control group $(\mathrm{P}<0.001)$. Moreover, for every $10 \%$ increase in CMA, there was a $5.4 \%$ decrease in the odds of suicide death after adjusting for demographic characteristics and substance use disorders.

Conclusion: Patients who died by suicide were less likely to be adherent to antidepressants than controls. In addition, increased antidepressant adherence was associated with decreased risk of suicide death. These findings suggest that adherence to antidepressants may reduce risk of suicide death among patients with depression.

P12.04

\section{Rise and Regional Variance of Schedule II Stimulant Use in the United States}

\section{Sneha Vaddadi, ${ }^{1}$ Nicholas Czelatka, ${ }^{1}$ Belsy Gutierrez, ${ }^{2}$ Carlos Torres-Teran, ${ }^{3}$ Daniel Tron, ${ }^{2}$ Holly Funk, ${ }^{1}$ Susannah Pitt, ${ }^{1}$ Alison Varano, ${ }^{1}$ John Boyle, ${ }^{1}$ Bhumika Maddineni, ${ }^{4}$ Daniel Kaufman, ${ }^{1}$ Christen Ogden, ${ }^{1}$ Kenneth McCall, ${ }^{5}$ Brian Piper $^{1}$}

${ }^{1}$ Geisinger Commonwealth School of Medicine, Scranton, PA; '2University of Scranton, Scranton, PA; ${ }^{3}$ Misericordia University, Dallas, $P A ;{ }^{4}$ Geisinger Health, Danville, PA; 5 University of New England, Biddeford, ME

Background: Attention deficit hyperactivity disorder (ADHD) is a common neuropsychiatric disorder afflicting millions of children and adults, increasing in prevalence. As stimulants are a common treatment modality for children and adults with this condition, there is a great need to better understand trends of stimulant usage in the past several years. This report examines the pharmacoepidemiology of 3 Schedule II stimulants in the United States and evaluates national patterns of use from 2017 to prior years.
Methods: Data was extracted from the Automated Reports and Consolidated Ordering Systems (ARCOS) database for transactions involving amphetamine, methylphenidate, and lisdexamfetamine. Data concerning total grams per drug were averaged across all states and compared from 2010 to 2017. Based on 2018 stimulant orders from Geisinger Health records, median values for daily dosage ( $\mathrm{mg} / \mathrm{person} /$ day) per drug were determined and used to compare stimulant usage trends across several years. For all data, outliers were determined through a Grubbs analysis and excluded. Data was averaged and compared with an unpaired $t$-test.

Results: Initial analysis of total grams averaged across all states indicates a $67.5 \%$ increase in amphetamine and a $76.7 \%$ increase in lisdexamfetamine use from 2010 to 2017. A change in methylphenidate was not significant. The rise in lisdexamfetamine and amphetamine use was supported with a daily-dose and population-considered analysis per state. Further analysis indicated that persons/day usage of stimulants in the West was lower than that of other U.S. regions from 2014 to 2017. A social variable analysis indicated a negative correlation between the percentage of Hispanic population per state and the daily dose/population per stimulant. Additional analyses of the 2018 data are ongoing.

Conclusion: The increasing trend of amphetamine and lisdexamfetamine use that is not seen in methylphenidate use may be explained by a rise in adult ADHD diagnoses and treatment. Regional analysis with daily dose indicates that the use of stimulants in the West may be distinct from that in other regions. A social variable analysis indicated lower stimulant use in areas with greater Hispanic population, possibly due to socioeconomic factors. Further research is being done with 2018 ARCOS data to investigate ongoing trends as well as social factors driving stimulant use and regional variance.

\section{P12.05}

\section{The Association Between Suicide Mortality and Weight Change}

Leah Hecht, ${ }^{1}$ Hseuh-Han Yeh, ${ }^{1}$ Lisa Miller-Matero, ${ }^{1}$ Anjali Thakrar, ${ }^{1}$ Shivali Patel, ${ }^{1}$ Gregory Simon, ${ }^{2}$ Frances Lynch, ${ }^{3}$ Arne Beck, ${ }^{4}$ Ashli Owen-Smith, ${ }^{5}$ Rebecca Rossom, ${ }^{6}$ Beth Waitzfelder, ${ }^{7}$ Christine Y. Lu, ${ }^{8}$ Jennifer M. Boggs ${ }^{4}$ Brian Ahmedani ${ }^{1}$

${ }^{1}$ Henry Ford Health System, Detroit, MI; ${ }^{2}$ Kaiser Permanente Washington, Seattle, WA; ${ }^{3}$ Kaiser Permanente Northwest, Portland, OR; ${ }^{4}$ Kaiser Permanente Colorado, Denver, CO; ${ }^{5}$ Kaiser Permanente Georgia, Atlanta, GA; ${ }^{6}$ HealthPartners, Bloomington, $\mathrm{MN}$; ${ }^{7}$ Kaiser Permanente Hawaii, Honolulu, HI; ${ }^{8}$ Harvard Pilgrim Health Care, Boston, MA

Background: There is an inverse association between body mass index (BMI) and death by suicide. Weight fluctuation has been associated with disease-related mortality, and some evidence suggests self-reported unexplained weight loss is 
linked with suicide mortality among men. However, existing research has not examined associations between objective weight change and suicide mortality among men and women, and existing research suffers from low power due to small sample size. Examining this physiological marker in a larger sample may provide a more robust base from which providers can confidently detect individuals at risk for suicide.

Methods: A case-control study was conducted to examine the relation between change in BMI during the previous year and suicide mortality. Cases $(\mathrm{n}=373$ who died by suicide) and controls $(n=1865)$ with 2 or more recorded weights in the previous year were derived from data from 7 Mental Health Research Network-affiliated health care systems.

Results: Cases were predominantly male (71\%) and Caucasian (69\%), with a mean age of 57 years, as compared to controls (42\% male; 60\% Caucasian; mean age: 42 years). Most cases (79\%) had a mental/substance use disorder diagnosis and a Charlson score of $>0$ (57\%). Change in BMI measured 7-12 months prior to index date (the date of suicide death) to that measured 0-6 months prior differed significantly between cases (mean: $0.52 \mathrm{~kg} / \mathrm{m}^{2}$ decrease, standard deviation [SD]: 1.86) and controls (mean 0.16 $\mathrm{kg} / \mathrm{m}^{2}$ increase, SD: $\left.4.52 ; \mathrm{P}<0.0001\right)$. One unit decrease in BMI was associated with increased risk of suicide mortality (odds ratio: 0.87, 95\% CI: 0.83-0.92; $\mathrm{P}<0.0001$ ). This association remained significant after adjusting for demographic characteristics, diagnoses of mental disorders, and Charlson comorbidity index (adjusted odds ratio: 0.93, 95\% CI: 0.88-0.98; $\mathrm{P}=0.0097)$.

Conclusion: In the year prior to suicide, weight loss was associated with suicide mortality even after accounting for physical and mental health indicators. Health care providers should evaluate weight change as a physiological marker and recognize weight loss may indicate or confer elevated risk for suicide mortality. Intervening when weight loss is observed may help to prevent suicide among high-risk patients.

\section{P12.06}

\section{Applying the Agile Implementation Method to a Pilot Study Addressing Low-Risk Chest Pain and Anxiety in the Emergency Department Setting}

Jill Nault Connors, ${ }^{1}$ Kurt Kroenke, ${ }^{1}$ Patrick Monahan, ${ }^{1}$ Paul Musey, ${ }^{1}$ Yelena Chernyak, ${ }^{1}$ Emily Holmes, ${ }^{1}$ Marshall Conley, ${ }^{2}$ Steve Roumpf, ${ }^{3}$ Parveen Chand, ${ }^{3}$ Malaz Boustani ${ }^{1}$

${ }^{1}$ Indiana University, Bloomington, IN; ${ }^{2}$ lead patient partner; ${ }^{3}$ Indiana University Health, Indianapolis, IN

Background: Chest pain is one of the most common chief complaints seen in emergency departments (ED); however, only about $20 \%$ of these patients have a true cardiopulmonary emergency. Undiagnosed or untreated anxiety is the likely underlying cause of chest pain and related symptoms in up to half of the $80 \%$ of patients deemed low risk for a major adverse cardiac event. However, current care in the ED is focused on ruling out life-threatening cardiopulmonary events and rarely is a discussion of anxiety initiated. This represents a significant missed opportunity to initiate treatment for anxiety among patients who present to the ED with low-risk chest pain and anxiety (LRCP-A).

Methods: Agile implementation is a comprehensive and cohesive model for identifying and implementing evidence-based solutions to health care delivery problems. These models draw on the theories and frameworks from behavioral economics, complex adaptive systems, and social networks. Agile implementation has been successfully applied to multiple health care challenges related to Alzheimer's disease care, intensive care unit survivorship, and central line-associated bloodstream infections. In this study, the agile implementation model is applied to implementation of screening, brief intervention, and referral to treatment (SBIRT) for anxiety among LRCP-A patients seeking care in the emergency department setting. The agile implementation method entails an 8-step process that includes: 1) Identify opportunities and confirm demand for the solution; 2) Identify evidence-based (or innovative) solutions; 3) Develop evaluation and termination plans; 4) Assemble a team to develop the minimally viable service; 5) Perform implementation sprints; 6) Monitor implementation; 7) Monitor whole system performance; and 8) Develop a minimally standard operating procedure.

Results: Results will include presentation of multiple agile implementation process tools (eg, rubric for assessing demand) and their scoring, lessons learned during implementation sprints, and outcome results of the evaluation plan.

Conclusion: This study can demonstrate the feasibility for successful implementation of SBIRT for LRCP-A in the ED. Due to the pragmatism of the solution, findings from the study have a high potential for real-world adoption. In addition, SBIRT could potentially serve as a comparator for future studies that incorporate referral directly to evidencebased psychological treatments.

\section{P12.07}

Variation in Diagnosis and Prescription Practices in Pediatric Primary Care Within a Large Rural Health System

\section{Sean O'Dell, ${ }^{1}$ Anna Baker, ${ }^{1}$ Erin Van Enkevort, ${ }^{1}$ Amanda Young, ${ }^{1}$ Matthew Gass, ${ }^{1}$ Victoria Schlieder ${ }^{1}$ \\ ${ }^{1}$ Geisinger Health, Danville, PA}

Background: Few studies have investigated system-level practice characteristics of pediatricians, and no known studies have included predominantly rural populations. This project investigated variation in diagnosis and prescription practices within a large rural health system. 
Methods: A retrospective review of electronic health record data was conducted in 28 rural practices for patients, 4-18 years of age, with 1 or more office visits from January 1, 2009, to December 31, 2017. To examine the roles availability of child psychiatrists, colocation of mental health professionals, and foster care prevalence have on diagnoses and prescription practices, separate generalized linear mixed models with a logit link were run. Practice characteristics were included as fixed effects and practice site was included as a random intercept to account for the relatedness of patients within each clinic. All models included sex, age, and the interactions between practice characteristics and patient age and sex in the models.

Results: Among 78,762 youth, $13.68 \%$ received a mental health diagnosis and $27.97 \%$ were prescribed psychotropic medication. Attention deficit hyperactivity disorder was the most common disorder diagnosed (7.8\%), followed by anxiety $(5.3 \%)$ and depression $(2.19 \%)$. Of note, $10.1 \%$ had more than 1 medication prescribed, with selective serotonin reuptake inhibitors (SSRI, 11.24\%), anxiolytics (13.74\%), and stimulants (9.32\%) being the most common. Colocated behavioral health services were associated with increased identification of mental health disorders and reduced proportion of youth with multiple medications prescribed. Stimulants were less common in colocated clinics (odds ratio: 0.65), whereas SSRIs (odds ratio: $1.54)$ and alpha agonists were more commonly prescribed (odds ratio: 1.32). Age and sex were frequently significant interactions; however, patterns were not evident across diagnoses and prescriptions. Community socioeconomic deprivation was not a significant predictor of diagnosis or prescription practices.

Conclusion: Compared with prior studies in mostly urban and suburban youth, this rural health system evidenced comparable identification of mental health disorders. However, psychiatric medications overall, multiple medications, and off-label prescriptions were more prevalent. These results indicate possible targets for de-implementation and warrant further investigation of the role colocated behavioral health services may play in practice variation.

\section{MULTIPLE CHRONIC CONDITIONS AND AGING POPULATIONS}

\section{OA5.01 \\ Prescribing Cascades in Patients With Alzheimer's Disease: Engaging Patients, Caregivers, Payers, and Providers}

Sarah Bloomstone, ${ }^{1}$ Kathryn Anzuoni, ${ }^{1}$ Noelle Cocoros, ${ }^{2}$ Jerry Gurwitz, ${ }^{1}$ Kevin Haynes, ${ }^{3}$ Vinit Nair, ${ }^{4}$ Richard Platt, ${ }^{2}$ Paula Rochon, ${ }^{5}$ Sonal Singh, ${ }^{1}$ Kathleen M. Mazor ${ }^{1}$
${ }^{1}$ Reliant Medical Group/University of Massachusetts Medical School/Fallon Health, Worcester, MA; ${ }^{2}$ Harvard Pilgrim Health Care, Boston, MA; ${ }^{3}$ HealthCore Inc., Wilmington, DE; ${ }^{4}$ Humana Inc., Louisville, KY; ${ }^{5}$ Women's College Research Institute, Toronto, Canada

Background: Prescribing cascades occur when a health care provider misinterprets a side effect as a new medical condition and prescribes a second, potentially unnecessary drug to address the side effect. Prescribing cascades can increase symptom burden and adversely affect healthrelated quality of life. Patients with Alzheimer's disease and related dementia (ADRD) are at increased risk for prescribing cascades due to high levels of multimorbidity, polypharmacy, and complexity of care. Educating patients, caregivers, and providers about prescribing cascades could reduce cascades and improve medication safety. We engaged patients, caregivers, providers, stakeholders, and experts to help develop informational materials that would stimulate patient/caregiver-provider conversations about prescribing cascades.

Methods: We recruited patients with ADRD, their caregivers, and providers from primary care and geriatric practices at an academic medical center in Massachusetts to provide feedback on draft materials. We also solicited feedback from expert advisors and stakeholders. We used an iterative approach - revising materials, soliciting feedback, and revising again. We mailed a follow-up survey to caregivers and providers to gather feedback on the revised materials. We coded transcripts of interviews and meetings using content analysis.

Results: Qualitative analysis of transcripts from interviews $(\mathrm{n}=14$ patient/caregiver; $\mathrm{n}=15$ provider) and meetings with stakeholders and advisors $(\mathrm{n}=5)$ highlighted several challenges: 1) reaching caregivers, as materials would be addressed to patients; 2) explaining the prescribing cascade concept clearly; 3 ) capturing providers' attention; and 4) framing recommendations for providers to be both actionable and respectful of clinical judgment. Caregivers and providers expressed concern about the impact of the materials on the encounter (eg, time) and relationship (eg, trust), but also saw value in educating caregivers. Some providers noted engaging caregivers to raise questions would be an effective way to gain their attention. Providers found the revised materials informative (11 of 11) and actionable (10 of 11), and caregivers found them understandable (9 of 10 ) and useful (10 of 10).

Conclusion: Engaging patients, caregivers, providers, stakeholders, and experts during the development of informational materials on prescribing cascades identified several challenges and ultimately resulted in materials that providers considered informative and actionable and that caregivers judged understandable and useful. The effectiveness of these materials in reducing prescribing cascades will be tested in a future trial. 


\section{OA5.02 \\ Medication Therapy Problems Identified Through Comprehensive Medication Management by Pharmacists in a Team-Based At-Home Care Program}

Margo Graybill, ${ }^{1}$ Vanessa Duboski, ${ }^{1}$ Eric Wright, ${ }^{1}$ Melissa Kern, ${ }^{1}$ Carrie Blanchard, ${ }^{2}$ Leeann Webster, ${ }^{1}$ Sarah Krahe-Dombrowski, ${ }^{1}$ Theron Ward, ${ }^{1}$ Michael Gionfriddo ${ }^{1}$

${ }^{1}$ Geisinger Health, Danville, PA; ${ }^{2}$ Center for Medication Optimization, UNC Eshelman School of Pharmacy, Chapel Hill, NC

Background: To control rising health care costs and improve quality, health care systems have designed approaches to care within the home for patients who have high levels of health care utilization. Pharmacists, as part of a multidisciplinary team within these programs, provide a variety of services to patients including comprehensive medication management (CMM), a collaborative patientcentered approach to medication optimization. Within CMM, pharmacists identify and resolve medication therapy problems (MTPs). The extent and impact on MTP identification and resolution among pharmacists functioning within multidisciplinary teams as part of an athome program has not previously been described.

Methods: Five pharmacists, practicing telephonically, currently support 3 at-home multidisciplinary teams across Geisinger, an integrated health delivery system in Pennsylvania. All pharmacists completed didactic and experiential training, along with fidelity and peer-review quality assurance assessments for the performance of CMM. Electronic health record notes were constructed with Smart data elements to record MTPs and document consultations, actions, follow-up, and resolutions of MTPs. The categorization of MTPs was guided by a framework developed by the Pharmacy Quality Alliance. We completed a retrospective descriptive study of the MTPs identified from within CMM electronic notes taken between December 12, 2018, and June 21, 2019. Descriptive statistics were run using Microsoft Excel to determine the quantity of MTPs as well as the acceptance rates of recommendations.

Results: We identified 232 patients who received CMM. On average, pharmacists identified 4 MTPs per patient, with a $35 \%$ acceptance rate (317 of 909). Of those accepted, pharmacists were able to resolve $142(45 \%)$ of these MTPs on their own while providers resolved the remaining 175 (55\%) MTPs. The most common MTP category was safety, accounting for $44 \%$ of the total MTPs. The least common MTP category was adherence, accounting for only $6 \%$ of the total MTPs identified.

Conclusion: Pharmacists are valuable members of the health care team and identified several MTPs when conducting CMM within a team-based at-home care program. These
MTPs, however, often fail to be resolved. Future studies should examine ways to improve intervention acceptance as well as the clinical and economic impact of changes made as a result of CMM and MTP identification.

P4.01

Geriatrics Research Instrument Library (GRIL): A Comprehensive Online Resource for Guiding Instrument Selection for Research in Older Adults

Kathryn Anzuoni, ${ }^{1}$ Christopher Delude, ${ }^{1}$ Mayra Tisminetzky, ${ }^{1}$ Heather Allore, ${ }^{2}$ Peter Charpentier, ${ }^{2}$ John Hepler, ${ }^{3}$ Dalane Kitzman, ${ }^{3}$ Gail McAvay, ${ }^{2}$ Michael Miller, ${ }^{3}$ Nicholas Pajewski, ${ }^{3}$ Jerry Gurwitz ${ }^{1}$

${ }^{1}$ Reliant Medical Group/University of Massachusetts Medical School/Fallon Health, Worcester, MA; ${ }^{2}$ Yale School of Medicine, New Haven, CT; ${ }^{3}$ Wake Forest School of Medicine, Winston-Salem, NC

Background: The Geriatrics Research Instrument Library (GRIL), an online resource of data collection instruments commonly used in gerontological research, has been revised and updated by the Advancing Geriatrics Infrastructure and Network Growth (AGING) Initiative in collaboration with the Older Americans Independence Centers (OAIC) Coordinating Center and the Yale OAIC. Clinical studies of older adults need appropriate and validated instruments, which often differ from those used in younger adults.

Methods: GRIL is systematically inventoried and updates are made to instruments, domains, and features on an ongoing basis. Instruments are included based on expert feedback by domain using systematic instrument reviews and instruments submitted by users. GRIL was programmed into the newly developed National Institute on Aging OAIC website with a platform utilizing Microsoft SQL 2017 database and an Adobe ColdFusion 2019 application server. Results: Thus far, 116 instruments and 18 domains comprise GRIL. Instrument characteristics such as time to completion, mode of implementation, and validity in older adults are incorporated. The new web-based platform includes userfriendly features and tracking statistics and can be accessed by GRIL collaborators from the different networks to be easily managed collaboratively.

Conclusion: GRIL provides a user-friendly public resource to aid researchers in efficiently choosing appropriate instruments to measure clinical outcomes in older adults across different settings. By leveraging resources and documenting a systematic, low-burden process to curate GRIL, invested organizations can create a sustainable resource over time. Evaluation measures will be developed to demonstrate GRIL's utility and to implement future expansion of this important resource. 
P4.02

\section{Predictors of Glycemic Relapse in Adults With Type 2 Diabetes}

JoAnn Sperl-Hillen, ${ }^{1}$ Shannon McKearnan, ${ }^{1}$ Patrick J. O'Connor, ${ }^{1}$ Jay Desai, ${ }^{1}$ Lilian Chumba, ${ }^{1}$ Karen L. Margolis, ${ }^{1}$ Stephanie Hooker, ${ }^{1}$ Rebecca Rossom, ${ }^{1}$ Gabriela Vazquez-Benitez, ${ }^{1}$ Malini DeSilva ${ }^{1}$

\section{${ }^{1}$ HealthPartners, Bloomington, $M N$}

Background: Diabetes quality improvement stops when the proportion of diabetes patients falling out of control in a defined time period (relapse vector) equals or exceeds the proportion of diabetes patients that are coming under control (improvement vector). In high-performing care systems the relapse vector is now acting as a brake on diabetes quality improvement. Strategies to proactively identify and manage diabetes patients at high risk for glycemic relapse are urgently needed.

Methods: Purpose of this study was 1) to identify characteristics of patients with type 2 diabetes (T2D) who experience "glycemic relapse," defined as transitioning from being in A1c goal $(<8 \%)$ to out of goal $(\geq 8 \%)$ within the subsequent year; and 2) to develop and compare 3 prediction models that quantify a given patient's risk of near-term glycemic relapse. Eligible T2D patients had an index visit between January 1, 2017, and May 31, 2018, with baseline A1c of $<8 \%$ within 3 months before the index visit, and at least 1 more A1c reading within 12 months of index visit. Glycemic relapse was defined as any post-index visit A1c of $\geq 8 \%$ within 12 months. Prediction models for glycemic relapse, using least absolute shrinkage and selection operator (GL-LASS) logistic regression and random forest (GL-RF), were compared to logistic regression with only index A1c (GL-I). We evaluated the discriminatory performance using area under the receiver operating characteristic curve (AUC), net reclassification, and positive predictive value at a risk threshold of 0.60 , using a 10 -fold cross-validation approach.

Results: Of 25,236 eligible T2D patients, $20.25 \%$ experienced A1c relapse; median time to relapse was 9.2 months (interquartile range: 6.3-11.3 months). The GLLASSO model included age, gender, race, hypoglycemia, microvascular comorbidities, glucose-lowering medications, index A1c value, and pre-index A1c value. Cross-validated AUCs were 0.80 for GL-I, 0.82 for GL-LASSO, and 0.81 for GL-RF. Net reclassification index was 0.04 for GLLASSO. Positive predictive value was 0.62 for GL-LASSO, as compared to 0.59 for GL-I.

Conclusion: All 3 prediction models adequately identified adults with T2D who have high risk of near-term glycemic relapse, with GL-LASSO performing best. Proactive approaches to preventing glycemic relapse should be considered in high-performing care systems.
P4.03

Health Care Utilization in the Year Following NonMuscle-Invasive Bladder Cancer Diagnosis Among Older Adults With Multiple Chronic Conditions

\author{
Tullika Garg, ${ }^{1}$ Andrea Berger, ${ }^{1}$ H. Lester Kirchner ${ }^{1}$
}

${ }^{1}$ Geisinger Health, Danville, PA

Background: Two-thirds of older adults with cancer have 2 or more chronic conditions, ie, multiple chronic conditions (MCC). Non-muscle-invasive bladder cancer (NMIBC) affects older adults and is a burdensome chronic condition in itself, requiring frequent outpatient visits and surgery. Frequent visits may not align with the goals of medically complex older patients with cancer, but little is known about how NMIBC diagnosis impacts downstream health care utilization. The objective was to describe health care utilization following NMIBC diagnosis by MCC status.

Methods: We identified older adults (age of $\geq 60$ years) diagnosed with NMIBC (stage of $<$ II) between 2003 and 2015. Health care utilization was defined as the number of contact days in the form of outpatient visits, inpatient days, and emergency department visits. Multiple visits on the same day counted as one contact day. We applied Agency of Healthcare Research and Quality's Clinical Classifications Software and Chronic Condition Indicator tools to outpatient and inpatient diagnosis codes to identify chronic conditions. MCC was defined as 2 or more chronic conditions. A multivariable linear regression model adjusted for age and prior cancer diagnosis was used to estimate the difference in health care utilization in the year prior to and following NMIBC diagnosis.

Results: Of 317 patients with NMIBC, 263 (83\%) had MCC. Patients with MCC were older (74 years vs 68.6 years), more often had prior cancers ( $29.7 \%$ vs $14.8 \%)$, and had more prescribed medications (7 vs 2). Patients with NMIBC and MCC had higher utilization prior to diagnosis (14 days vs 2 days). Patients without MCC had a larger change in utilization following NMIBC diagnosis, with an average increase of 13.9 days compared to 7.5 days in the MCC group. In the multivariable model, patients without MCC had an increase of 6.23 days, more than those with MCC (95\% CI: 1.34-11.12, P=0.01).

Conclusion: Following NMIBC diagnosis, health care utilization increased in older adults. Older adults without MCC had larger increases in health care utilization than those with MCC. Adding a new chronic condition such as NMIBC increases the volume of health care work that older adults perform. These data may serve as a baseline for future studies to reduce burdens related to NMIBC diagnosis and treatment in medically complex older adults. 
P4.04

Non-Cardiac-Related Morbidity, Mobility Limitation, and Outcomes in Older Adults With Heart Failure

Mayra Tisminetzky, ${ }^{1}$ Jerry Gurwitz, ${ }^{1}$ Hassan Fouayzi, ${ }^{1}$ Robert Goldberg, ${ }^{1}$ Dongjie Fan, ${ }^{2}$ Sue Hee Sung, ${ }^{2}$ Alan Go, ${ }^{2}$ Kristi Reynolds, ${ }^{3}$ David H. Smith ${ }^{4}$

${ }^{1}$ Reliant Medical Group/University of Massachusetts Medical School/Fallon Health, Worcester, MA; ${ }^{2}$ Kaiser Permanente Northern California, Oakland, CA; ${ }^{3}$ Kaiser Permanente Southern California, Pasadena, CA; ${ }^{4}$ Kaiser Permanente Northwest, Portland, OR

Background: To examine the individual and combined associations of non-cardiac-related conditions and mobility limitation with morbidity and mortality in adults with heart failure.

Methods: We conducted a retrospective cohort study in a large diverse group of adults with heart failure from 5 U.S. integrated health care delivery systems. We characterized patients with respect to the presence of noncardiac conditions ( $<3$ vs $\geq 3$ ) and/or mobility impairment (defined by the use/nonuse of a wheelchair, cane, or walker), categorizing them into 4 subgroups. Outcomes included all-cause death and hospitalizations for heart failure or any cause.

Results: Study population comprised 114,553 adults diagnosed with heart failure (mean age: 73 years; $46 \%$ women). Compared to those with $<3$ noncardiac conditions and no mobility limitation, adjusted hazard ratios for allcause death among those with $<3$ noncardiac conditions and mobility limitation, those with $\geq 3$ noncardiac conditions and no mobility limitation, and those with $\geq 3$ noncardiac conditions and mobility limitation were 1.40 (95\% CI: $1.31-$ 1.51), 1.72 (95\% CI: 1.69-1.75), and 1.93 (95\% CI: $1.85-$ 2.01 ), respectively. We did not observe an increased risk of any-cause or heart failure-related hospitalization related to the presence of mobility limitation among those with a greater burden of noncardiac multimorbidity. Consistent findings regarding mortality were observed within groups defined according to age, gender, and heart failure type (preserved, reduced, midrange ejection fraction), with the most prominent impact of mobility limitation in those less than 65 years of age.

Conclusion: There is an additive association of mobility limitation — beyond the burden of noncardiac multimorbidity - on mortality for patients with heart failure, and this association is especially prominent in younger patients.
P4.05

Patient and Caregiver Perspectives on Engagement in Primary Care

Eleanor Rivera, ${ }^{1}$ Karen Hirschman, ${ }^{1}$ Justine Sefcik, ${ }^{2}$ Kathy McCauley, ${ }^{1}$ Alexandra Hanlon, ${ }^{3}$ Mark Pauly, ${ }^{1}$ Matthew Press, ${ }^{1}$ Mary Naylor ${ }^{1}$

${ }^{1}$ University of Pennsylvania, Philadelphia, PA; ${ }^{2}$ Drexel University, Philadelphia, PA; ${ }^{3}$ Virginia Tech, Blacksburg, VA

Background: Effective care management is recognized as essential to high-quality outcomes among diverse, often complex patients whose needs are often addressed by multiple health care and social service organizations. Primary care plays a pivotal role in the provision of care management services, yet little is known about patients' experiences with these services. The purpose of this study was to explore the experiences of older adult patients and family caregivers accessing and engaging with primary care services where care management is offered.

Methods: A purposive sample of patients and caregivers who were members of patient and family advisory councils (PFACs) at 6 Medicare-designated Comprehensive Primary Care Plus practices affiliated with a large academic health system in southeastern Pennsylvania and New Jersey were invited to participate in a single telephone interview about their experiences with their primary care practice and services provided. All interviews were digitally recorded, transcribed, reviewed for completeness, and then coded using conventional content analysis techniques. Coded data were organized in NVivo 12 based on the relevant codes and then collapsed into common themes.

Results: A total of 30 PFAC members participated. All participants were patients of the practices they represented, with 1 also being a caregiver and 2 being a married couple. Participants were mostly female and, on average, 72 years old with 9 chronic conditions. Patients and caregivers described their impressions of how engaged they are in decisions about their care (themes: adherence, goal-setting, self-triage, proactive); their perceptions of their health care providers and quality of health care services (themes: accessibility, competence, caring, time spent); their experiences with their clinicians and how they work with them to focus on goals and preferences and preparation to manage health problems (themes: communication quality, guidance); and their perceptions about the primary care practice as the "go to" place (compared to the emergency department) for issues related to care (themes: care team, convenience, electronic health record). Overall findings support patient engagement and general satisfaction with care.

Conclusion: These patient and caregiver experiences and perspectives on care management and primary care illustrate that patient satisfaction can align closely with existing quality metrics. 


\section{P4.06}

Design and Pilot Test of a Primary Care-Based Trial of Deprescribing for Persons With Cognitive Impairment and Multimorbidity

Elizabeth Bayliss, ${ }^{1}$ Leslie Wright, ${ }^{1}$ Shetterly Susan, ${ }^{1}$ Jonathan Norton, ${ }^{2}$ Kathy Gleason, ${ }^{1}$ Mahesh Maiyani, ${ }^{1}$ Emily Reeve, ${ }^{3}$ Matthew Maciejewski, ${ }^{4}$ Orla Sheehan, ${ }^{2}$ Jennifer Wolff, ${ }^{2}$ Ariel Green, ${ }^{2}$ Courtney Kraus, ${ }^{1}$ Linda Weffald, ${ }^{1}$ Melanie Drace,${ }^{1}$ Cynthia Boyd ${ }^{2}$

${ }^{1}$ Kaiser Permanente Colorado, Denver, CO; 2 Johns Hopkins University, Baltimore, MD; ${ }^{3}$ University of South Australia, Adelaide, Australia; ${ }^{4}$ Duke University, Durham, NC

Background: Most individuals with cognitive impairment have multimorbidity and associated polypharmacy, placing them at risk for adverse health outcomes. Optimizing medications through deprescribing (reducing or stopping the use of inappropriate medications or medications unlikely to be beneficial) may improve outcomes for this population, but optimal approaches to deprescribing remain unclear. Our goal was to design and test a pragmatic, primary care-based, patient-centered intervention to optimize prescribing for older adults with cognitive impairment and multimorbidity.

Methods: The study consisted of qualitative stakeholder engagement followed by a pilot test of the intervention. It was conducted in a not-for-profit, integrated delivery system targeting adults age 65 or older with cognitive impairment, multimorbidity, and 5 or more chronic medications. Intervention design was informed by interviews with patient/ family member dyads $(n=4)$, primary care clinicians $(n=6)$, a patient/family advisory panel, and a clinician advisory panel prior to the pilot. After the pilot, additional input was obtained from 4 patient/family interviews and a clinician focus group to inform intervention refinement. The 4-month pilot intervention included two parts: an informational brochure and short questionnaire mailed to patients prior to primary care clinic visits to encourage patient engagement; and a short initial presentation followed by periodic 1-page "tip sheets" provided to clinicians to increase awareness about deprescribing processes and communication. Primary pilot outcomes were feasibility and acceptability.

Results: During intervention development, stakeholders emphasized the importance of lay language, continuity relationships, and a longitudinal approach to deprescribing. During the pilot intervention, 58 patients received materials and 15 clinicians received 6 tip sheets. In debriefing interviews, patients/family members found the intervention to be acceptable and were receptive to deprescribing conversations. They recommended materials be sent twice over 12 months. Clinicians found the tip sheets to be useful and requested additional "pearls" for deprescribing. Of 20 random postpilot chart reviews, 5 revealed medication discontinuation or deintensification activities during the pilot period.
Conclusion: A pragmatic, primary care-based deprescribing intervention designed with stakeholder input is feasible and acceptable. A cluster randomized pragmatic trial will assess the effect of a refined intervention on optimal prescribing for patients with cognitive impairment.

\section{P4.07}

Prediabetes Awareness, Clinical Risk Factors, Lifestyle Behaviors, and Provider Experience in Rural and Nonrural Patients in a Midwestern Health Care System

Jay Desai, ${ }^{1}$ Daniel Saman, ${ }^{2}$ Jacob Haapala, ${ }^{1}$ A. Lauren Crain, ${ }^{1}$ Clayton Allen, ${ }^{2}$ Kris Ohsorg, ${ }^{1}$ Patrick J. O'Connor, ${ }^{1}$ JoAnn Sperl-Hillen, ${ }^{1}$ Steven Dehmer ${ }^{1}$

\section{${ }^{1}$ HealthPartners, Bloomington, MN; ${ }^{2}$ Essentia Health,} Duluth, MN

Background: Prediabetes impacts over 84 million Americans, but few studies have examined the burden of prediabetes within rural communities.

Methods: In 2016, as part of an intervention trial, we conducted web-based surveys among patients 18-72 years of age without diabetes and who had a hemoglobin A1c of 5.7\%$6.4 \%$. Patients attended clinics in Rural (small town/rural) or Nonrural (metropolitan/micropolitan) commuting areas. Survey measures included sociodemographic and clinical characteristics, health status and well-being, lifestyle behaviors, and provider engagement. Key risk factors (body mass index, blood pressure, tobacco use, aspirin use), laboratory data (hemoglobin A1c, lipids), and comorbid diagnosis data will be collected from the electronic medical record.

Results: Of the 1528 patients contacted, 122 did not meet intervention trial eligibility criteria, 708 refused, 698 consented, and 550 completed the survey. Participant demographics were similar between the Rural and Nonrural participants. Among participants, awareness of prediabetes was $40 \%$; $78 \%$ reported a blood glucose test in the past year; and 57\% reported high blood pressure. About 10\% of participants reported 5+ daily servings of fruits and vegetables. Almost $24 \%$ of Nonrural participants reported daily consumption of 1+ sugar-sweetened beverage compared to $15 \%$ of Rural participants. Similarly, $40 \%$ of Nonrural participants reported eating $2+$ times/week at a fast food or sit-down restaurant compared to $24 \%$ of Rural participants. Approximately 39\% reported $150+$ minutes/week of leisure-time physical activity, and $22 \%$ reported strength training $2+$ days/week. Daily sedentary time was 11.5 hours compared to 10.3 hours among Nonrural and Rural participants, respectively. Satisfaction with providers ranged from $71 \%$ for knowing about patient history to $82 \%$ for demonstrating respect for what patients had to say. Provider discussions about blood glucose, weight control, healthy eating, and physical activity was around $60 \%$ among participants but was lower for cardiovascular and 
diabetes risk at $37 \%$ and $49 \%$, respectively. Collection of electronic medical record data is in progress.

Conclusion: Rural participants had some more favorable physical activity and nutrition practices, but there is a substantial gap in prediabetes awareness and management in both groups. Results suggest that increased clinician discussion of prediabetes and its management, and greater integration of patient-reported behaviors into primary care shared decisionmaking are areas of interest for future intervention studies.

\section{P4.08}

Prevalence of Medication Adherence Issues in Patients Not Achieving Recommended Care Goals

JoAnn Sperl-Hillen, ${ }^{1}$ Jeffrey Anderson, ${ }^{1}$ Jacob Haapala, ${ }^{1}$ Lilian Chumba, ${ }^{1}$ Heidi L. Ekstrom, ${ }^{1}$ Deepa Appana, ${ }^{1}$ Malini DeSilva, ${ }^{1}$ Patrick J. O'Connor ${ }^{1}$

\section{${ }^{1}$ HealthPartners, Bloomington, $M N$}

Background: As part of a pragmatic trial of a clinical decision support (CDS) intervention to improve medication adherence for diabetes, hypertension, and hyperlipidemia, we first collected data on the prevalence of medication adherence issues.

Methods: In two large care systems in Minnesota, 28 clinics were randomized to an adherence CDS intervention or control. The CDS intervention used web-based algorithms to identify people $18-75$ years old who met one of the following clinical criteria and also had an objective adherence issue using an Epic-based measurement of proportion of days covered (ePDC): 1) A1c of $\geq 8 \%$ with a noninsulin glycemic medication ePDC of $<80 \%$; 2) blood pressure (BP) of $\geq 140 / 90$ on 2 separate consecutive dates with a BP medication ePDC of $<80 \%$; and 3) ACC/AHA criteria for statin use and low-density lipoprotein of $>70 \mathrm{mg} / \mathrm{dl}$ with statin ePDC of $<80 \%$. The ePDC was calculated within Epic using SureScripts dispense and claims data. For the first 6 weeks prior to beginning the adherence intervention, August 7, 2019-September 17, 2019, the CDS was run silently in all clinics to quantify the number of patients who would be eligible to receive the adherence CDS and pharmacist outreach intervention.

Results: Of 55,377 patients, age 18-75, with 1 or more encounters in the 6-week period, a BP medication was identified in 20,181 (BP Cohort). Of these, 2363 met the criteria for not being at clinical goal and 1452 also had ePDC of $<80 \%(61 \%)$. A noninsulin diabetes medication was identified in 6573 (Diabetes Cohort). Of these, 1701 met the criteria for not being at clinical goal and 1095 also had ePDC of $<80 \%(64 \%)$. A statin was identified in 14,171 (Lipid Cohort). Of these, 8674 met the clinical criteria for lipids and 4098 also had ePDC of $<80 \%$ (47\%). Of 2069 patients who met all the study inclusion and exclusion criteria, 12\% were eligible for more than 1 cohort.
Conclusion: Rates of medication adherence issues are high among patients who are not meeting recommended care goals for blood pressure, diabetes, and lipids. No problems are anticipated for accrual rates of study-eligible patients or power to detect clinically significant changes in medication adherence and clinical outcomes.

\section{P4.09}

\section{User-Centered Decision Support: Formative Provider-Usability Evaluation of Integrated Clinical Decision Support for Chronic Kidney Disease Care and Management in Primary Care}

Pritika Kumar, ${ }^{1}$ Stephanie Freund, ${ }^{1}$ Deepika Appana, ${ }^{1}$ Lilian Chumba, ${ }^{1}$ Patrick J. O'Connor, ${ }^{1}$ JoAnn SperlHillen ${ }^{1}$

\section{${ }^{1}$ HealthPartners, Bloomington, MN}

Background: To prevent serious chronic kidney disease (CKD) complications such as end-stage renal disease and cardiovascular events, better strategies are needed to identify, treat, and refer patients with CKD who are seen in primary care clinics. The CKD-Wizard (CW) is an addition to an existing and successful nonproprietary web-based clinical decision support system, called Priorities Wizard, and includes key elements of CKD care for patients seen in primary care settings, including recognition of CKD, management of blood pressure and glucose, and timely referral to nephrologists when appropriate. Our objective is to formatively evaluate $\mathrm{CW}$ that not only helps clinicians plan treatment protocols with a comprehensive understanding of the patients' comorbid diagnoses but also promotes evidence-based conversations between patients and clinicians regarding patient-specific risk and patients' specific concerns.

Methods: User-centered design with practice-based and participatory decision aid development will be used to design, develop, and evaluate patient-centered decision support regarding CKD care in primary care clinics within the HealthPartners (HP) Medical Group. User experience and user interface (UX/UI) development will involve successive iterations with incremental refinement in 4 phases: 1) initial prototype development; 2) usability assessment; 3) field testing; and 4) beta testing. This qualitative approach will involve input from the HP patient advisory board members, primary care clinicians, health services researchers, designers, and programmers at every stage.

Results: The CW will be a product of successive iterative revisions in accordance with UX/UI industry design standards. This will result in a useful and usable final product that integrates clinical decision support with a patient decision aid to promote shared decision-making between the primary care physician and patients. This 
electronic health record-integrated tool will facilitate evidence-based conversations regarding CKD in patients living with other comorbid conditions.

Conclusion: The $\mathrm{CW}$ is designed to align evidence-based practices about CKD in primary care patients. It aims to build trust, empower active participation by the patients about their condition, and promote shared decision-making between the primary care physicians and patients. We hypothesize that, when implemented, the $\mathrm{CW}$ will support the clinician-patient relationship, effectively manage CKD (especially so for patients with other comorbid conditions), and improve the patient experience of care.

\section{P4.10}

\section{Efficacy of a Nitric Oxide Dressing in Decreasing Bacterial Counts on Human Skin}

Rachel Nye, ${ }^{1}$ Kristi Robinia, ${ }^{1}$ Josh Sharp, ${ }^{1}$ Paul Peterson $^{1}$

\section{${ }^{1}$ Northern Michigan University, Marquette, MI}

Background: The presence of infection contributes to prolonged and costlier hospitalizations and may even cause death. Surgical site infections, blood stream infections, and antimicrobial resistance are global health problems. 1,2,3,4 nitric oxide (NO) use is a potential strategy for preventing skin infections, which may be implicated in blood stream infections and antibiotic use.

Methods: Three dressings containing the experimental compound (NO) as well as 3 control dressings were applied without antiseptic preparation to intact skin on the backs of 66 healthy volunteers. Baseline microflora bacterial counts were obtained on day 3 of the study. Subsequent skin cultures were obtained underneath dressing pairs ( 1 experimental and 1 control) on days 3, 5, and 7 after application. A within-subjects design using a sample of convenience was completed to compare the efficacy of bacterial reduction under the experimental and control dressings.

Results: On each removal day the experimental NO dressing demonstrated a statistically significant reduction ( $\mathrm{P}$-values ranged from $<0.001$ to $<0.007$ ) in bacterial count when compared to the control dressing. In summary, the NO compound film dressings had significantly lower bacterial colony counts as compared to the control Tegaderm ${ }^{\circledR}$ on days 3,5 , and 7 .

Conclusion: Incorporating a nitric oxide compound into a dressing has an antibacterial effect lasting a minimum of 7 days. This dressing has the potential to prevent skin infection, thereby potentially reducing cost and length of stay.

\section{CANCER PREVENTION, TREATMENT \& CARE}

OA6.01

\section{Validation of an Algorithm to Identify History of Cancer Using Electronic Medical Records}

Jennifer C. Gander, ${ }^{1}$ Mahesh Maiyani, ${ }^{2}$ Brooke Thompson, ${ }^{2}$ Drew Bradlyn, ${ }^{1}$ Stacey Honda, ${ }^{3}$ Cyndee Yonehara, ${ }^{3}$ Katrina Goddard, ${ }^{3,4}$ Melanie Francisco, ${ }^{3,4}$ Robert T. Greenlee, ${ }^{5}$ Habibul Ahsan, ${ }^{6}$ Muhammad G. Kibriya, ${ }^{6}$ Brisa Aschebrook-Kilfoy, ${ }^{6}$ Christina L. Clarke, ${ }^{2}$ Heather Spencer Feigelson ${ }^{2}$

${ }^{1}$ Kaiser Permanente Georgia, Atlanta, GA; ${ }^{2}$ Kaiser Permanente Colorado, Denver, CO; ${ }^{3}$ Kaiser Permanente Hawaii, Honolulu, HI; ${ }^{4}$ Kaiser Permanente Northwest, Portland, OR; ${ }^{5}$ Marshfield Clinic, Marshfield, WI; ${ }^{6}$ University of Chicago, Chicago, IL

Background: In partnership with the National Cancer Institute, we are developing a prospective cohort of adults, 40-65 years old, to study cancer etiology and prevention (https://dceg.cancer.gov/research/who-we-study/cohorts/ connect). For this cohort, it is imperative to enroll a cancerfree population. While tumor registries are the "gold standard" to identify incident cancers, tumor registries may not include recent diagnoses (within the past 12-18 months) or a history of cancer prior to health plan enrollment. An algorithm developed in 2013 from Kaiser Permanente (KP) Colorado's electronic medical record combined tumor registry data and diagnosis codes to achieve a $100 \%$ sensitivity and $84.6 \%$ specificity for identifying prior cancers, although it has not been validated in other health care settings. Our goal is to update this "history of cancer" algorithm and validate it across 6 geographically diverse health plans (HP).

Methods: We included all 2016 members between the ages of 40 and 65 years from the participating sites to ensure the tumor registry was complete at each participating health plan. Patients who ever had an inpatient or outpatient diagnosis code or ever had an observation in the tumor registry were flagged as having a history of cancer. In situ cancer diagnosis codes, previously excluded from the 2013 algorithm, were included. Each health plan will validate the algorithm through chart review $(n=200)$ and calculate the sensitivity, specificity, positive predictive value, and negative predictive value.

Results: A total of 529,254 members from 4 health plans are included in our preliminary findings: KP Colorado $(\mathrm{n}=177,515), \mathrm{KP}$ Georgia $(\mathrm{n}=104,638)$, KP Hawaii $(\mathrm{n}=76,910)$, and KP Northwest $(n=170,191)$. The algorithm identified an average of $91.5 \%$ cancer-free adults. Averages per site were 90.3\% for KP Colorado, $93.0 \%$ for KP Georgia, $93.0 \%$ for KP Hawaii, and $90.1 \%$ for KP Northwest. Across sites, there were more cancer-free individuals who were younger and male. Marshfield Clinic and University of Chicago also will 
be participating in the algorithm validation. Results from validation of the algorithm at all health plans is underway.

Conclusion: A validated algorithm to identify cancer cases that may fall outside of tumor registries may be a useful tool in a variety of analytic settings. Understanding how the algorithm performs in different health plans will help optimize the process for broad dissemination.

\section{OA6.02}

Adherence to Provider Referrals for Lung Cancer Screening With Low-Dose Computed Tomography

\author{
Jiang Li, ${ }^{1}$ Meghan Martinez, ${ }^{1}$ Harold S. Luft ${ }^{1}$ \\ ${ }^{1}$ Sutter Health, Walnut Creek, CA
}

Background: In 2013, guidelines for lung cancer screening (LCS) with low-dose computer tomography (LDCT) were released and LCS-LDCT became a Medicare-covered benefit in 2015. Despite this, LCS-LDCT uptake remains low, in part because LCS-LDCT is a complex multistep process that requires considerable effort on the part of the patient, provider, and health system. Older heavy smokers who are eligible for LCS-LDCT may face barriers such as inconvenience, perceived smoking-related stigma, and distrust of the health care system, which may lead to decreased LCS-LDCT participation. At the system level, there is no unified way to schedule, track, or ensure patients complete screenings or follow-ups. Instead, each department has their own processes, systems, and workflows, which may influence uptake of LCS-LDCT in clinical settings. This study aims to examine trends in adherence to provider referrals for LCS-LDCT and the multilevel factors influencing completion rate of LCSLDCT orders in one health care system following a series of guideline/policy changes.

Methods: We analyzed electronic health record data from a large health care system in Northern California between January 2010 and May 2019. A total of 46,100 LCS-LDCT orders were included in the analysis. For any patient with an LCS-LDCT order, trends in the proportion of eligible vs ineligible patients (per screening guidelines) and screening completion rates were assessed and compared. Multilevel factors associated with completion of the LCS-LDCT order were explored using hierarchical generalized linear models.

Results: About $40 \%$ of patients referred for LCS-LDCT did not meet all eligibility criteria for screening. Completion rate varies from the lowest ( $2 \%$ in 2014) before Medicare coverage to the highest (63\% in 2018) after Medicare coverage. Completion rates vary significantly across providers $(\tau \tau 00$ : 1.4261, standard error: 0.06141). Approximately 25.4\% of variability in the LCS-LDCT completion rate is accounted for by the providers, leaving $74.6 \%$ of the variability to be accounted for by the patients or other unknown factors. Older age and being ineligible for LCS-LDCT are factors that negatively impact LCS-LDCT completion. Those who visited a provider with more patients referred to LCS-LDCT are more likely to complete the screening test.

Conclusion: This abstract is considered "work in progress" to be completed by April 2020. Our findings on the barriers and facilitators to LCS-LDCT screening recommendations are likely to influence clinical practice at patient, provider, and systems levels to inform targeted interventions.

OA6.03

\section{Implementing Universal Lynch Syndrome Screening Programs Across Three Health Care Systems: A Qualitative Cross-Case Comparison}

Alanna Kulchak Rahm, ${ }^{1}$ Zachary M. Salvati, ${ }^{1}$ Victoria Schlieder, ${ }^{1}$ Jamie Atondo, ${ }^{1}$ Ilene Ladd, ${ }^{1}$ Deborah Cragun, ${ }^{2}$ Jessica Ezzell Hunter, ${ }^{3}$ Mara M. Epstein, ${ }^{4}$ Jan Lowery, ${ }^{5}$ Laurie Jenson, ${ }^{6}$ Christine Y. Lu, ${ }^{7}$ Pamala A. Pawloski, ${ }^{8}$ Ravi Sharaf, ${ }^{9}$ Su-Ying Liang, ${ }^{10}$ Andrea Burnett-Hartmann, ${ }^{11}$ Marc S. Williams ${ }^{1}$

${ }^{1}$ Geisinger Health, Danville, PA; ${ }^{2}$ University of South Florida, Tampa, FL; ${ }^{3}$ Kaiser Permanente Northwest, Portland, OR; ${ }^{4}$ Reliant Medical Group/University of Massachusetts Medical School/Fallon Health, Worcester, MA; ${ }^{5}$ University of Colorado, Aurora, CO; ${ }^{6}$ Catholic Health Initiatives, Englewood, CO; ${ }^{7}$ Harvard Pilgrim Health Care, Boston, MA; ${ }^{8}$ HealthPartners, Bloomington, $\mathrm{MN}$; ${ }^{9}$ Weill Cornell Medicine, New York, NY; ${ }^{10}$ Sutter Health, Walnut Creek, CA; ${ }^{11}$ Kaiser Permanente Colorado, Denver, CO

Background: Despite multiple guidelines, implementation of universal tumor screening (UTS) for Lynch syndrome (LS) in health care systems remains variable. The IMPULSS study was created to better understand and use this variability to guide implementation in a variety of health care settings.

Methods: Nine health care systems (7 HCSRN, 1 academic, 1 national system) were purposively selected for variability in implementing UTS for the IMPULSS study (2 with UTS, 1 without UTS). To date, stakeholder interviews have been completed across 3 organizations ( 2 HCSRN, 1 non-HCSRN). Multiple stakeholders at each organization, including leaders, oncologists, genetic counselors, and gastroenterologists, participated in semi-structured interviews guided by the Consolidated Framework for Implementation Research (CFIR). Interviews were audio-recorded and transcribed for coding and analysis. Within each organization, we coded organization data relevant to the CFIR domains of inner setting, process, characteristics of individuals, and intervention characteristics.

Results: We completed 16 stakeholder interviews across 3 organizations. Respondents included 7 genetic counselors/ genetics nurses, 3 administrators/clinician leaders, 2 oncologists, 1 pathologist, and 3 other clinicians (family practice, pharmacy). Using a qualitative cross-case comparison 
approach, similar implementation challenges were identified across all 3 organizations related to structural characteristics, resources, communication, and champions. Factors reported as important to initial program implementation were internal pathology and leadership support, while availability of systematic processes and tracking were reported as important to program optimization. Intraorganizational variability in UTS implementation also was uncovered. Within one organization reporting UTS implementation, interviews revealed UTS was implemented only at locations with internal pathology. Within the organization without UTS, stakeholders reported implementing tumor screening in one location via a clinical trial (not specific to LS identification). Conclusion: Findings of intra-organizational variability in UTS implementation illuminates additional complexities in health care systems due to heterogeneity in organizational structures and availability of resources within organizations. These preliminary data demonstrate that implementation may need to be characterized differently within organizations to understand barriers and facilitators accurately, and that additional facilitating factors may be found in unrelated programs indirectly achieving the desired practice. These findings also confirm the importance of viewing implementation as an ongoing process that is best facilitated through the lens of opportunities within local contexts.

\section{OA6.04 \\ Associations Between Patient and Tumor Characteristics and Screen-Detected Versus Symptomatic Colon Cancers in Two Integrated Health Care Systems}

\author{
Elizabeth Sarma, ${ }^{1}$ Matthew Thompson, ${ }^{2}$ Erin Bowles, ${ }^{3}$ \\ Andrea Burnett-Hartman, ${ }^{4}$ Lu Chen, ${ }^{3}$ Rebecca \\ Hubbard, ${ }^{5}$ Onchee $\mathrm{Yu}^{3}{ }^{3}$ Jessica Chubak ${ }^{3}$ \\ ${ }^{1}$ National Cancer Institute, Bethesda, MD; ${ }^{2}$ University \\ of Washington, Seattle, WA; ${ }^{3}$ Kaiser Permanente \\ Washington, Seattle, WA; ${ }^{4}$ Kaiser Permanente \\ Colorado, Denver, CO; ${ }^{5}$ University of Pennsylvania, \\ Philadelphia, PA
}

Background: Colon cancer screening is credited with reducing colon cancer mortality, but little is known about the characteristics of patients and tumors of screendetected colon cancers compared to colon cancers that are detected symptomatically. Our objective was to describe the proportions of colon cancer diagnoses detected by screening or symptomatic presentation and to assess patient and tumor characteristics associated with these diagnosis routes in two integrated health care delivery systems.

Methods: We conducted a study among adults, age 50 to 85 years, with stage I-IIIA colon cancer diagnosed during 1995-2014 at Kaiser Permanente Colorado or Kaiser Permanente Washington. Mode of detection (screen-detected/ asymptomatic or symptomatic) was ascertained from medical records. Multivariable logistic regression models were used to estimate odds ratios (OR) and $95 \%$ confidence intervals (CI) comparing the odds of screen-detected colon cancer by patient factors - demographics at diagnosis (year, age, sex, race, smoking status, body mass index [BMI], Charlson score), prediagnosis primary care utilization, and disease factors (stage, location) - adjusted for included factors and study site.

Results: Of 1675 colon cancer patients, $38.4 \%$ had screen-detected colon cancer and $61.6 \%$ were detected symptomatically. Patients more likely to have a screen-detected diagnosis were diagnosed during 2010-2014 vs 1995-1999 (OR: 1.57, 95\% CI: 1.07-2.30), were male (OR: 1.30, 95\% CI: $1.04-1.63$ ), had a BMI of $25-<30 \mathrm{~kg} / \mathrm{m}^{2}$ (OR: $1.36,95 \%$ CI: $1.04-1.80$ ) or $30+\mathrm{kg} / \mathrm{m}^{2}$ (OR: $1.39,95 \%$ CI: $1.05-1.85$ ) vs $<25 \mathrm{~kg} / \mathrm{m}^{2}$, and had transverse vs left-side cancer (OR: 1.66, 95\% CI: 1.10-2.50). Relative to patients with no comorbidities, patients with 1 (OR: $0.72,95 \%$ CI: 0.55-0.95) or $2+$ comorbidities (OR: $0.35,95 \%$ CI: $0.25-0.49$ ) at the time of diagnosis were less likely to have a screen-detected cancer, as were patients diagnosed with stage IIA (OR: 0.34, 95\% CI: $0.27-0.43$ ) or IIB (OR: $0.12,95 \%$ CI: $0.06-0.24$ ) relative to stage I.

Conclusion: The majority of colon cancers were diagnosed following symptomatic presentation, but the proportion that were screen-detected increased over time. Certain patients, particularly those with more comorbidities and higher-stage cancers, are less likely to have a screendetected diagnosis, even after adjusting for health care utilization. Additional research is needed to increase colon cancer screening in patients likely to benefit from it.

\section{OA11.01 \\ Development of an Electronic Intervention Tool in Epic for the Ambulatory Stem Cell Transplant Pharmacist}

\section{Benjamin Andrick, ${ }^{1}$ Lorraine Tusing, ${ }^{1}$ Joseph Vadakara, ${ }^{1}$ Christina Gregor ${ }^{1}$ \\ ${ }^{1}$ Geisinger Health, Danville, PA}

Background: National guidelines recommend pharmacists be included as part of the multidisciplinary care team for patients undergoing stem cell transplant. While previous efforts have sought to justify their role through metrics such as inpatient services and outpatient prescription retention, evaluations of the ambulatory stem cell transplant pharmacists' direct impact remain uncertain. We describe the development and implementation of an electronic pharmacist intervention tool smart phrase in Epic to capture and quantify ambulatory interventions by the stem cell transplant pharmacist.

Methods: The pharmacist intervention tool was designed based on the Pharmacy Quality Alliance's Medication Therapy Problem Categories Framework. The intent of this framework was to utilize nationally recognized standard 
definitions for benchmarking. The intervention tool documents the medication or disease status on which the pharmacist intervened as well as classification of the intervention and magnitude of the encounter. The pharmacist intervention tool was built into Epic using standard data elements incorporated through a smart phrase. Stem cell transplant pharmacists were then educated to incorporate the smart phrase as a part of their routine documentation of direct patient care.

Results: The pharmacist intervention tool was implemented in May 2019, and interim evaluation of the tool was conducted in October 2019. The pharmacist intervention tool was used in 662 unique encounters across 47 stem cell transplant patients, the majority of whom underwent allogeneic stem cell transplant (97\%). An average of 1.56 medications was managed per encounter, and the pharmacist addressed an average of 1.44 medication-related problems per encounter. The most frequently managed issues included cyclosporine (328), tacrolimus (17), and disease (14). Pharmacists most often made 2.25 interventions per encounter. The most frequently documented inventions were monitoring (354), graft-vs-host-disease surveillance (185), infection surveillance (152), and immunosuppression management (69).

Conclusion: A standard data element-based smart phrase can be utilized to document interventions of the ambulatory stem cell transplant pharmacist. This pharmacist intervention tool allows for quantification of the pharmacist's direct impact on patient care versus other surrogate outcomes. Future analysis should incorporate such pharmacist intervention data to elucidate the value to the stem cell transplant patient in the ambulatory setting.

\section{OA11.02}

\section{Implementation of Objective Assessment of Swallow Function Before and After Treatment of Head and Neck Cancers}

\section{Samantha $\operatorname{Tam}^{1}$}

\section{${ }^{1}$ Henry Ford Health System, Detroit, MI}

Background: Management of head and neck cancer is complicated due to the debilitating functional impairments often accompanying the primary disease and/or related therapeutic interventions. Unfortunately, there are limited data objectively describing change in functional swallowing after the treatment of head and neck cancer. To this end, we utilized an objective measure of swallow function, the Functional Oral Intake Scale (FOIS), to investigate the impact and further define the prognosis for head and neck cancer treatment.

Methods: Retrospective chart review was performed analyzing data from 219 patients with diagnosed head and neck cancer. Patients underwent pretreatment and posttreatment swallow studies, in which swallow function was assessed and categorized using the FOIS. The FOIS classifies swallowing capabilities by examining oral and pharyngeal phases of swallowing on a scale from 1 to 7 . A score of 7 indicates no functional swallowing deficit, whereas a 1 indicates no oral intake ability. Scores of 1-3 are feeding tube-dependent, whereas scores of 4-7 can receive oral preparations to various degrees. Paired $t$-tests were used to detect changes in swallow function from pretreatment to posttreatment.

Results: Overall, head and neck cancer treatment resulted in a statistically significant decrease in FOIS score from 6.3 to $4.6(\mathrm{P}<0.0001)$. For stage IV squamous cell carcinoma $(\mathrm{n}=126)$, FOIS decreased from 6.2 to $4.3(\mathrm{P}<0.0001) ; 19 \%$ $(n=24)$ required a feeding tube 1 -year posttreatment. For stage III squamous cell carcinoma $(\mathrm{n}=33)$, FOIS scores trended downward from 5.8 to $5.1(\mathrm{P}=0.26) ; 6 \%(\mathrm{n}=2)$ required a feeding tube 1-year posttreatment. For stage II squamous cell carcinoma $(\mathrm{n}=35)$, FOIS decreased from 6.4 to $4.8(\mathrm{P}<0.001)$; $9 \%(n=3)$ required a feeding tube 1 -year posttreatment. For stage I squamous cell carcinoma $(\mathrm{n}=25)$, FOIS scores trended downward from 6.8 to $5.5(\mathrm{P}=0.09) ; 8 \%(\mathrm{n}=2)$ required a feeding tube 1-year posttreatment.

Conclusion: The treatment of head and neck cancer resulted in significant decreases in FOIS. Additionally, patients with stage IV squamous cell carcinoma experience the most substantial decline in swallow function, with a higher need for a feeding tube 1-year posttreatment. This study demonstrates the first pre- and posttreatment objective assessment of swallow studies for patient undergoing head and neck cancer treatment.

\section{OA11.03}

Cost-Effectiveness of Upfront Maintenance Therapies in Advanced Ovarian Cancer: Does It Work for Everyone or Targeted Patients Only?

\author{
Su-Ying Liang, ${ }^{1}$ Michael Richardson, ${ }^{2}$ Daniel S. Kapp, ${ }^{2}$ \\ Amandeep Mann, ${ }^{1}$ John Chan ${ }^{1}$
}

\section{${ }^{1}$ Sutter Health, Walnut Creek, CA; ${ }^{2}$ Stanford University, Stanford, CA}

Background: Several new maintenance therapies with antivascular and poly (ADP-ribose) polymerase (PARP) inhibitors (bevacizumab, niraparib, and olaparib) for ovarian cancer have been approved by the U.S. Food and Drug Administration in recent years. While economic analysis of bevacizumab has been conducted, relatively little is known about niraparib and olaparib due to insufficient effectiveness data. Taking advantage of effectiveness data from clinical trials that recently became available in September 2019, this study evaluates cost-effectiveness of maintenance therapies in advanced ovarian cancer, with a specific focus on niraparib and olaparib.

Methods: Data on clinical outcomes, including progressionfree survival (PFS), were obtained from clinical trials (PRIMA and PAOLA-1). Cost data were obtained from our previous work and the Centers for Medicare \& Medicaid drug payment and physician fee schedule. We developed a Markov model and computed the incremental cost-effectiveness ratios 
(ICERs) of maintenance therapies relative to placebo. We conducted stratified analyses to assess variations in ICERs across subpopulations (eg, homologous recombination [HR] status: deficient, proficient, or undetermined; biomarker status: BRCA-muted [BRCAm] or wild-type). Sensitivity analyses were performed to evaluate impacts of key parameters, particularly drug costs, on ICERs.

Results: Based on estimated costs of niraparib (200 mg at $\$ 9883$ per month over 3 years of maintenance) and 5.6 months PFS gained for the overall stage III-IV ovarian cancer patients, 12 months PFS gained for BRCAm patients, and 11.5 months PFS gained for patients who were HR-deficient, the incremental costs per progression-free life-year gained were \$758,546, \$353,988, and \$369,379, respectively. Based on estimated costs of olaparib $(\$ 16,178$ per month for 2 years of maintenance, with bevacizumab $\$ 9775$ per month for 21 months) and 5.5 months PFS gained for the overall sample, 20 months PFS gained for BRCAm patients, and 11 months PFS gained for HR-deficient patients, the incremental costs per progression-free life-year gained were $\$ 837,818$, $\$ 236,308$, and $\$ 418,909$, respectively. In comparison, the ICER of bevacizumab was $\$ 416,051$ with 6 months PFS. A Markov model incorporating quality of life is under development, and estimates of ICERs will be updated.

Conclusion: Our preliminary findings suggest that benefits of the maintenance therapies vary across the overall sample and subgroups. Maintenance with targeted therapies in advanced ovarian cancer can be cost-effective, particularly with companion biomarker guidance.

\section{OA11.04}

\section{Conversations About Goals of Care: Insights From Interviews With Patients With Advanced Cancer and Oncology and Palliative Care Teams}

\author{
Ellis C. Dillon, ${ }^{1}$ Amy Meehan, ${ }^{1}$ Steve Lai, ${ }^{1}$ Su-Ying \\ Liang, ${ }^{1}$ Natalia Colocci, ${ }^{1}$ Julie Roth, ${ }^{1}$ Harold S. Luft, ${ }^{1}$ \\ Catherine Nasrallah ${ }^{1}$

\section{${ }^{1}$ Sutter Health, Walnut Creek, CA}

Background: There is widespread agreement that patientcentered care begins with understanding patients' goals and values. There are concerns that individuals with advanced cancer receive serious illness and end-of-life care that is not aligned with their values and preferences. However, we lack in-depth information on how these goals are discussed in conversations between patients and clinicians.

Methods: In-depth interviews were conducted with a convenience sample of patients with advanced cancer and members of medical oncology, gynecological oncology, and palliative care teams in a large multispecialty group in Northern California. The research team conducted qualitative data analysis using open coding to identify emergent themes. Results: Of those contacted, 25 of $29(86 \%)$ patients and 25 of $38(66 \%)$ care team members (physicians, nurses, social workers, etc) completed interviews. Goals-of-care conversations arose at 2 time points: 1) at diagnosis of stage IV cancer or metastasis ("establishing goals of care"); and 2) when disease progressed or pain and symptoms became too difficult ("changing goals-of-care conversations"). Establishing goals-of-care conversations with oncology teams were about understanding the goal of treatment (to cure cancer, extend life, etc), and prognosis ("How much time do I have?"). Patients and oncologists varied in their desire to hear or give specific prognostic timelines, with some patients expressing ambivalence. Both patients and providers expressed beliefs about the fluidity of prognosis, particularly due to new cancer treatments. Changing goals-of-care conversations concerned stopping treatment, hospice care, prognostic awareness, quality of life, advance care planning, and end-of-life planning. Palliative care teams often facilitated these conversations, and patients appreciated discussing their options and preferences. Both patients and care teams acknowledged challenges, such as when to have these conversations. Some patients desired earlier conversations and palliative care involvement; others wanted to get through their first round of treatment or wait until things were "going downhill."

Conclusion: Both patients and care teams acknowledged the complexity around having goals-of-care conversations but desired improved communication to enhance goal-aligned care and improve patient and family end-of-life experiences. Patients reported that palliative care teams played an important role in enhancing changing goals-of-care conversations and decisions.

\section{P2.01}

\section{Economic Evaluation of Universal Lynch Syndrome Screening Strategies Among Newly Diagnosed Patients With Colorectal Cancer}

Jing Hao, ${ }^{1}$ Dina Hassen, ${ }^{1}$ James M. Gudgeon, ${ }^{2}$ Susan R. Snyder, ${ }^{3}$ Heather Hampel, ${ }^{4}$ Janet L. Williams, ${ }^{1}$ Marc S. Williams, ${ }^{1}$ Christine Y. Lu, ${ }^{5}$ Ravi N. Sharaf, ${ }^{6}$ Victoria Schlieder, ${ }^{1}$ Alanna Kulchak Rahm ${ }^{1}$

${ }^{1}$ Geisinger Health System, Danville, PA; ${ }^{2}$ Intermountain Healthcare, Salt Lake City, UT; ${ }^{3}$ Georgia State University, Atlanta, GA; ${ }^{4}$ Ohio State University Wexner Medical Center, Columbus, OH; ${ }^{5}$ Harvard Pilgrim Health Care, Boston, MA; ${ }^{6}$ Weill Cornell Medicine, New York, NY

Background: Lynch syndrome (LS) is the most common form of inherited colorectal cancer (CRC). Tumor screening for LS in all newly diagnosed CRC patients has the highest (tier 1) Centers for Disease Control and Prevention evidence rating and support for broad implementation in health care systems; however, implementation of universal LS screening remains suboptimal. The objective of this study is to compare the relative effectiveness and efficiency of different LS screening strategies among newly diagnosed $\mathrm{CRC}$ populations from the health care system perspective. 
Methods: Decision analytic models were created from previous work and updated to reflect new LS screening developments, including reduced genetic testing costs, new evidence on the value of double somatic tumor sequencing, and consideration of tumor sequencing as an upfront test. We developed decision analytic models representing 8 viable LS screening protocols for a hypothetical cohort of 1000 patients with newly diagnosed CRC. Model assumptions and parameter values were extracted from a literature review and augmented by input from clinical experts. Costs were mainly obtained from the Medicare fee schedule and are in 2018 U.S. dollars.

Results: Preliminary results based on the models of the first 7 protocols indicated the direct germline sequencing protocol provided the best sensitivity $(99.90 \%, 95 \%$ CI: 99.57\%-99.93\%) and equal specificity $(99.50 \%, 95 \% \mathrm{CI}$ : 97.28\%-99.85\%) in terms of identifying LS cases, compared to immunohistochemistry (IHC) (sensitivity: $79.33 \%, 95 \%$ CI: $72.17 \%-80.99 \%$; specificity: $99.98 \%, 95 \%$ CI: $99.88 \%-$ 99.99\%) and microsatellite instability (MSI) (sensitivity: 81.13\%, 95\% CI: $73.60 \%-82.91 \%$; specificity: $99.99 \%$, 95\% CI: $99.93 \%-100 \%$ ) protocols. The total program costs were $\$ 0.9 \mathrm{M}(95 \% \mathrm{CI}: \$ 0.5 \mathrm{M}-\$ 1.7 \mathrm{M})$ for direct germline sequencing, compared to $\$ 0.5 \mathrm{M}(95 \%$ CI: $\$ 0.4 \mathrm{M}-\$ 0.6 \mathrm{M})$ for IHC and \$0.6M (95\% CI: \$0.4M-\$0.7M) for MSI. Threshold analysis showed that the cost of the genetic sequencing panel to the health care system would need to be $\$ 376$ for direct germline sequencing to be as efficient as the IHC protocol, ie, have the same cost per LS case identified.

Conclusion: Direct germline sequencing was the most effective protocol for identifying LS cases but was more expensive than the IHC protocol, which had the lowest cost per LS case identified but misses more LS cases. Direct germline sequencing would be as efficient as the IHC protocol if the costs of the tests decline.

\section{P2.02}

Determinants of Atrial Fibrillation Development Among Patients Undergoing Ibrutinib Therapy

\section{Tinuade Piwuna ${ }^{1}$}

\section{${ }^{1}$ Marshfield Clinic Health System, Marshfield, WI}

Background: Within the last decade, the use of ibrutinib, a first-generation, nonselective, irreversible Burton's tyrosine kinase inhibitor for the treatment of hematologic malignancies has proved to be highly effective in improving patient outcomes. Although ibrutinib has been associated with an increase in atrial fibrillation (AF), yet the predisposing factors have not been well defined.

Methods: A retrospective approach was taken to identify patients diagnosed with hematologic (B-cell) malignancies and receiving ibrutinib therapy at Marshfield Clinic to evaluate the risk factors associated with the development of AF. The outcome of interest was the time to AF diagnosis after receiving ibrutinib therapy, and the age at $\mathrm{AF}$ was categorized into $<60,60-80$, and $>80$ years. Stepwise Cox regressions were employed to select vital predictors.

Results: 192 patients on ibrutinib therapy were recruited for the study; $65.6 \%$ were male, median age was 72 years ( 71.9 \pm 11.2 ), with $79 \%$ between 60 and 80 years, and $91 \%$ were of non-Hispanic white ethnicity. Of the 192 patients, 56 (29.2\%) developed AF, with 2 months as median time to AF. The first Cox model indicated age, prior AF history, and congenital heart disease as crucial predictors for $\mathrm{AF}$ outcome while on ibrutinib therapy. Patients who were $>80$ years old, with previous history of AF/coronary heart disease, were at the highest risk of developing $\mathrm{AF}$ at 12-month follow-up (hazard ratio [HR]: 11.7, 95\% CI: 6.5-21.2). In the second Cox model, a history of congestive cardiac failure (HR: $5.5,95 \%$ CI: 2.9 10.4), congenital heart disease (HR: 5.9, 95\% CI: 1.4-25.4), and hypertension (HR: 2.9, 95\% CI: 1.5-5.6) significantly predicted AF event, when age was not considered.

Conclusion: Our study illustrates the relationship of an increased risk in atrial fibrillation in elderly patients with cardiovascular comorbidities who receive ibrutinib therapy, indicating the significance of timely screening and intervention in populations at risk to improve patient outcomes. Our study is one of the first to identify congenital heart disease as a risk factor for the development of ibrutinib therapy-related AF.

P2.03

Impact of Race and Socioeconomic Status on Survival in Patients With Oropharyngeal Cancer

Samantha Tam, ${ }^{1}$ Ryan Freedman, ${ }^{2}$ Haley Sibley, ${ }^{1}$ Amy Williams, ${ }^{1}$ Steven Chang ${ }^{1}$

${ }^{1}$ Henry Ford Health System, Detroit, MI; ${ }^{2}$ Wayne State School of Medicine, Detroit, MI

Background: Due to human papillomavirus (HPV)related oropharyngeal carcinoma, its incidence is increasing significantly, despite the decrease of incidence of head and neck cancer at other subsites. While HPV status in oropharyngeal cancer is a known predictor of survival, the effect of race and socioeconomic status has not been investigated. This study aims to investigate the effect of race and socioeconomic status on survival in patients with oropharyngeal carcinoma.

Methods: This was a retrospective cohort study of African American and Caucasian American patients treated for squamous cell carcinoma of the oropharynx from 1991 to 2012. Univariate and multivariate Cox proportional hazards models were created to understand the relationship between race, socioeconomic status, and overall survival.

Results: A total of 192 patients were included in the study cohort; 104 patients $(54.2 \%)$ had tumors that were positive for HPV. Of the 192 patients, 68 (35.4\%) were African American compared to 124 (64.6\%) Caucasian Americans. After adjusting for HPV status, stage at diagnosis, median household income in patient zip code, and proportion of residents in 
patient zip code who did not complete high school, African American race continued to be an independent predictor of overall survival (adjusted hazard ratio: 2.58, 95\% CI: 1.26-5.26). Other markers of socioeconomic status (median household income and education) were no longer predictors of overall survival following multivariate adjustment.

Conclusion: Even after adjusting for HPV status, race continued to be an important predictor for overall survival in patients with oropharyngeal cancer. Understanding the drivers for this racial disparity may allow for improved treatment decision-making in African American patients with oropharyngeal carcinoma.

\section{P2.04}

Neoadjuvant Modified Docetaxel, Cisplatin, and Fluorouracil Integrated Into a Multimodal Treatment Approach in Nonmetastatic Esophageal Cancer: A Community Experience

Adedayo Onitilo, ${ }^{1}$ Trista Stankowski-Drengler, ${ }^{2}$ Oyewale Shiyanbola, ${ }^{3}$ Jessica Engel, ${ }^{1}$ Sabo Tanimu, ${ }^{1}$ Ya-Huei Li ${ }^{1}$

\section{${ }^{1}$ Marshfield Clinic, Marshfield, WI; ${ }^{2}$ University of Wisconsin School of Medicine and Public Health, Madison, WI; '3niversity of Wisconsin-Madison, Madison, WI}

Background: A modified docetaxel, cisplatin, and fluorouracil $(\mathrm{mDCF})$ regimen as the first-line treatment for metastatic adenocarcinoma has been associated with higher rates of disease-free survival (DFS) and overall survival and lower rates of toxicities compared to DCF in the clinical trial setting. Yet, whether mDCF incorporated into a multimodal treatment approach will benefit patients with gastroesophageal cancer remains unknown.

Methods: A retrospective approach was taken to study the benefit and risk of adapting mDCF neoadjuvant into multimodal treatment for patients with nonmetastatic esophagus/gastroesophageal junction cancer (NMEGJC) in the community setting. Patients diagnosed with NMEGJC between August 2008 and November 2017 and prescribed $\mathrm{mDCF}$ as adjuvant along with other chemotherapies, surgeries, and/or radiotherapy were identified for retrospective review. $\mathrm{mDCF}$ consists of docetaxel $40 \mathrm{mg} / \mathrm{m}^{2}$ intravenous (IV) infusion (day 1), cisplatin $40 \mathrm{mg} / \mathrm{m}^{2} \mathrm{IV}$ (day 3), fluorouracil $400 \mathrm{mg} / \mathrm{m}^{2}$ IV (day 1), fluorouracil $1000 \mathrm{mg} / \mathrm{m}^{2}$ continuous IV infusion over 48 hours (days 1 and 2), and leucovorin $400 \mathrm{mg} / \mathrm{m}^{2}$ (day 1). Outcomes of interest included diseasefree survival, overall survival, and hematologic toxicities. Descriptive statistics and Kaplan-Meier curves were performed using SAS 9.4.

Results: Thirty patients met inclusion criteria (median age: 64.9 years); $90 \%$ of the population was male. Of the $30,77 \%$ of NMEGJC had adenocarcinoma; $23 \%$ had squamous cell carcinoma. The majority had stage II or higher NMEGJC $(90 \%) ; 63.3 \%$ underwent esophagectomy. The 2-year and 5-year disease-free survival was $60.8 \%$ and $41.7 \%$, respectively, for adenocarcinoma and $71.4 \%$ and $71.4 \%$ for squamous cell carcinoma, respectively. The 2-year and 5-year overall survival was $64.9 \%$ and $44.5 \%$, respectively, for adenocarcinoma and $71.4 \%$ and $71.4 \%$ for squamous cell carcinoma, respectively. Both disease-free and overall survival decreased with increasing disease stage, histology (adenocarcinoma vs squamous), esophageal compared to gastroesophageal junction involvement, and without surgical intervention. The most frequent toxicity (Common Terminology Criteria for Adverse Events Version 5.0) for leukopenia (56.7\%) and thrombocytopenia (84.2\%) were grades I and II.

Conclusion: The mDCF neoadjuvant can be integrated into the multimodal treatment approach for stage II or higher NMEGJC as the first-line treatment because of noninferior survival rates and lower grade of toxicity compared to conventional perioperative regimens. Further studies with higher numbers of patients in different subtypes of carcinoma are urgently needed to confirm the preliminary findings.

\section{P2.05}

\section{Local Implementation of Cancer Care Delivery Research Within a Community NCORP}

\section{Pamala A. Pawloski, ${ }^{1}$ Heather Kehn, ${ }^{1}$ Alice Shapiro, ${ }^{2}$ Daniel Anderson, ${ }^{1}$ Michele Lacy, ${ }^{1}$ Mary Orsted ${ }^{1}$}

\section{${ }^{1}$ HealthPartners, Bloomington, MN; ${ }^{2}$ Park Nicollet Institute, Minneapolis, MN}

Background: Metro-Minnesota Community Oncology Research Consortium (MMCORC) is part of the National Cancer Institute Community Oncology Research Program (NCORP), a national network of cancer care investigators, providers, academia, and other organizations that care for diverse populations in health systems. MMCORC represents 28 hospitals and clinics in the Minneapolis, Minnesota, metropolitan area, suburbs, and rural locations, with more than 160 participating physician investigators. More than 600 patients are enrolled into clinical studies annually. The portfolio of clinical trials includes cancer care delivery research (CCDR). Our affiliate membership is the cornerstone of our CCDR program within MMCORC. The majority of affiliate member locations reside in rural locations within Minnesota and Wisconsin. Research coordinators at affiliate sites are employees of the health system and often have embedded roles within the cancer center. Affiliate sites are smaller due to lower patient volumes. Specialty departments already work closely together with established working relationships and single service line leadership.

Methods: Facilitators: Supportive providers introduce study to patient during clinic visit, followed by discussion with research coordinator. Use of the central screening innovation will alleviate burden on site research staff and target priority protocol needs. Collaboration will include patient advocates 
and community-based programs. Barriers include challenging clinic flow and timing of enrolling multiple patients with complex clinic visits within the protocol eligibility window. With nontraditional research partners, it takes an extra amount of time and effort to bring partners on board who were not previously part of Community NCORP research, such as pathology leaders, pharmacists, nurse navigators, lung screening departments, and surgical practices.

Results: Current number of open and enrolling care delivery protocols is 7 , number of enrolled patient participants to date is 100 , number of enrolled providers to date is 88 , and number of enrolled practices to date is 19 . MMCORC has 25 component sites, part of the 1000 study locations nationally.

Conclusion: Successful local implementation includes multidisciplinary leadership and a framework that provides resources to site staff who have engaged providers and working relationships with nontraditional research partners throughout the health system. Opportunities for growth include closely examining local cancer disparities and identifying care delivery research questions to address the issues.

\section{HEALTH EQUITY AND SOCIAL NEEDS}

\section{OA8.01 \\ Barriers to Implementing Social Needs Screening in Medical Care Settings: Findings From the National Academies of Sciences, Engineering, and Medicine}

\author{
Rachel Gold, ${ }^{1}$ Amber Haley, ${ }^{2}$ Lisa Cooper, ${ }^{3}$ Laura \\ Gottlieb $^{4}$
}

${ }^{1}$ Kaiser Permanente Center for Health Research, Portland, OR; '2University of North Carolina at Chapel Hill, Chapel Hill, NC; 3Johns Hopkins University, Baltimore, MD; ${ }^{4}$ University of California, San Francisco, San Francisco, CA

Background: A National Academies of Sciences, Engineering, and Medicine (NASEM) committee convened to examine the potential for integrating social needs care into the delivery of medical care. The committee was charged with assessing 1) current and emerging approaches to social needs care integration in diverse health care systems, 2) the roles of different disciplines, care organizations, and types of providers in the integration process, and 3) current and emerging efforts to inform the delivery of effective, efficient care that addresses social needs, improves health, and reduces health inequities. The committee's final report was released in September 2019. This presentation will provide an in-depth review of the report's findings on barriers to implementing social needs care integration and possible solutions to address these barriers.

Methods: The committee was comprised of 18 subject matter experts. A broad literature search was conducted, and 3 public sessions were held to obtain further information and perspectives. The committee met 6 times over an 18-month period to gather, review, and discuss the collected evidence before developing the conclusions and recommendations presented in the report.

Results: The identified implementation barriers associated with social needs care integration were grouped into 4 categories: barriers to initiating social care integration (eg, obtaining leadership support and staff buy-in, setting operational goals); documenting social needs (eg, identifying a target population, designing workflows, providing training, identifying appropriate screening methods); reviewing social needs (eg, identifying referral resources, creating/maintaining partnerships with social service providers); and responding to social needs (eg, developing workflows for internal referrals, identifying local resources for external referrals). The report proposes several strategies to address these identified barriers. Conclusion: National momentum for strengthening the intersection of social and medical care delivery is growing rapidly. However, efforts to implement social-medical care integration are likely to encounter implementation barriers. Findings from this NASEM report summarize the existing evidence and evidence gaps on potential barriers to social care integration as well as strategies that may support the implementation and sustainability of integrated medical and social care programming. Further research is needed to identify which implementation strategies will be most effective in different care settings.

\section{OA8.02}

Clinic Characteristics Associated With Social Determinants of Health Documentation in Community Health Centers

Erika Cottrell, ${ }^{1}$ Miguel Marino, ${ }^{2}$ Stuart Cowburn, ${ }^{1}$ Jorge Kaufmann, ${ }^{2}$ David Ezekiel-Herrera, ${ }^{2}$ Katie Dambrun, ${ }^{1}$ Arwen Bunce, ${ }^{1}$ Ned Mossman, ${ }^{1}$ Megan Hoopes, ${ }^{1}$ Molly Krancari, ${ }^{1}$ Rachel Gold ${ }^{3}$

${ }^{1}$ OCHIN, Inc., Portland, OR; ${ }^{2}$ Oregon Health \& Science University, Portland, OR; ${ }^{3}$ Kaiser Permanente Center for Health Research, Portland, OR

Background: Preliminary research suggests that electronic health record (EHR)-documented data on patients' social determinants of health $(\mathrm{SDH})$ can inform care teams about patient needs and facilitate referrals to social services. The need for standardized SDH data collection and response is arguably greatest in community health centers (CHCs), but little has been reported in implementation of SDH documentation in this important care setting.

Methods: We developed a suite of EHR tools for documenting patient-reported SDH data and implemented these tools in OCHIN's national network of CHCs in June 2016. In descriptive analyses on tool adoption, we used EHR data on the 581 clinics that had access to the tools at any time during the study period. We then conducted logistic regressions to 
model associations between SDH documentation and clinic characteristics among the 345 clinics that had access to the tools for the entire study period.

Results: In our descriptive analyses, 31,549 patients had a documented SDH screening from June 2016 to May 2018. The total number of screenings increased over time. Although $31 \%$ of eligible CHC clinics $(n=179)$ used the tool to document at least $1 \mathrm{SDH}$ screening, more than $50 \%$ of documented screenings occurred in just 4 of the $106 \mathrm{CHC}$ organizations, and more than half $(55 \%)$ of documented screenings only included responses for $1 \mathrm{SDH}$ domain. In our regression analyses, clinic-level analyses found no differences in SDH documentation by clinic rurality, percentage female, or racial/ethnic distribution of clinic patients. Clinics with a higher percentage of older patients had increased odds of SDH screening compared to those with younger populations. Clinics with higher rates of missing data on patients' federal poverty levels had decreased odds of SDH screening. Last, clinics with higher percentages of Medicaid or uninsured visits had lower odds of SDH screening.

Conclusion: With many national calls to document patients' $\mathrm{SDH}$ in the EHR, it is important to consider whether and how such screening varies across different kinds of clinics. These results suggest that national initiatives seeking to encourage EHR-based SDH documentation may need to account for clinic characteristics that may serve as barriers or facilitators to adopting this practice change.

\section{OA8.03}

The Potential for Electronic Health Record Digitized Text to Identify Indications of Social Needs That Can Influence Patient Health and Health Care Delivery

\author{
Douglas Roblin, ${ }^{1}$ Haihong $\mathrm{Hu},{ }^{1}$ Claudia $\mathrm{Nau}^{2}$
}

${ }^{1}$ Kaiser Permanente Mid-Atlantic States, Rockville, MD; ${ }^{2}$ Kaiser Permanente Southern California, Pasadena, CA

Background: Social determinants of health are increasingly relevant to the study of health care delivery and design of interventions to improve population health (eg, KP Thrive Local). There is little evidence or consensus on how best to measure social needs from the electronic health record (EHR). The objectives of our preliminary study were to 1) develop a straightforward SAS text-mining algorithm to identify indications of social risks and needs from free-text provider notes in a test population, and 2) assess the validity of this algorithm in identifying indications of social risks and needs. Methods: We reviewed more than 60,000 lines from EHR notes associated with the care of 6337 adults who were eligible for the Kaiser Permanente Mid-Atlantic States Complex Care Program during 2016-2018. We developed lists of keywords and phrases to indicate possible need for assistance in transportation, medication, housing, food/meal preparation, and utilities payment. The SAS algorithm scanned EHR notes for occurrences of text strings (eg, "cost of medication" or co-occurrence of "medication" and "assistance") and classified each patient over the 3 -year period into having or not having each type of social need. Data were linked with ICD-10-CM codes for social needs associated with health care encounters and with an area deprivation index describing the patients' residential Census block group.

Results: ICD-10-CM codes identified social needs for very few patients (eg, 1.3\% for "Z59.xx Housing and economic circumstances"). By comparison, the SAS algorithm identified many more patients with indications of need for assistance in housing (11.7\%), utilities payment $(22.8 \%)$, meals/food preparation $(56.9 \%)$, medication $(58.0 \%)$, and transportation (75.3\%). Patients with one social need were likely to have another social need $(\mathrm{P}<0.05)$. Social needs clustered within Census block groups (intraclass correlation coefficients of approximately $0.1(\mathrm{P}<0.05)$; higher percentages of patients with indications of social needs lived in areas of greater deprivation $(\mathrm{P}<0.05)$.

Conclusion: We found face and concurrent validity of a straightforward SAS algorithm for identifying indications of social needs in EHR notes. Our work establishes a framework for development of a natural language processing algorithm that can account for negation (eg, need assessed and ruled out) and context (eg, transportation assistance needed due to immobility vs lack of social support).

\section{OA8.04}

What Does the Unstructured Narrative Text in the Electronic Health Record Reveal About Social Influencers of Health?

\author{
David Chin, ${ }^{1}$ Terrence Habiyaremye, ${ }^{2}$ Vishal Shetty, ${ }^{1}$
} Melissa Kern, ${ }^{2}$ Eric Wright ${ }^{2}$

${ }^{1}$ School of Public Health and Health Sciences, University of Massachusetts Amherst, Amherst, MA; ${ }^{2}$ Geisinger Health, Danville, PA

Background: Social influencers of health $(\mathrm{SIoH})$ - the economic, educational, social, cultural, and physical environments in which patients live — are widely recognized to have a potent effect on a patient's health outcomes. Although validated instruments to collect SIoH exist, adoption of regular capture is challenging and limited. Clinical notes, the unstructured textual fields written by providers, provide another potential source of SIoH information, but these fields remain underexplored for SIoH information. We examined the prevalence and characteristics of markers for SIoH recorded in text narrative.

Methods: Patients, age 18 to 89 years, who had at least 5 encounters between June 1, 2018, and June 1, 2019, with at least 1 encounter with a primary diagnosis of major depression, within Geisinger Health, an integrated health care delivery system in Pennsylvania, were eligible. Among 2415 patients who met the inclusion criteria, 2000 were randomly selected for manual chart review by an abstractor. Unstructured text 
was abstracted from any clinical setting using a modified PRAPARE tool. We refined our abstraction tool using the first 15 records, excluded patients with more than 150 notes during the study period, and focused on 3 domains: 1) social interaction/connectivity; 2) social class; and 3) health behavior. Unstructured text was collected for these domains and independently annotated by a blinded abstractor.

Results: Among 100 patient records, we found 1693 unique textual strings relating to a SIoH domain. All charts (100 of 100) had at least 1 SIoH domain identified. SIoH textual strings were most prevalent among psychiatry $(27.8 \%)$, family medicine (19.0\%), and emergency medicine $(7.7 \%)$. Among 20 subdomains, 16 were identified in $\geq 30 \%$ of charts, with food insecurity, language, ethnicity, and poverty least reported.

Conclusion: SIoH are documented in unstructured textual narratives of patients with depression. We have built a conceptual model that represents some of the social and behavioral complexity of patients' lives that will serve as a roadmap for our long-term goal: the development of a natural language processing engine that retrospectively processes text, consolidating and presenting this knowledge to clinicians at the point-of-care. These findings provide some evidence of the potential for natural language processing implementation to understand $\mathrm{SIoH}$ in an integrated health care system.

\section{P9.01 \\ Examining the Association Between Social Isolation and Future Utilization Among the Elderly Population}

\author{
David Mosen, ${ }^{1}$ Matthew Banegas, ${ }^{1}$ Erin Keast, ${ }^{1}$ Briar \\ Ertz-Berger ${ }^{1}$
}

\section{${ }^{1}$ Kaiser Permanente Northwest, Portland, OR}

Background: Previous research has found that social isolation among individuals $65+$ years old may be associated with health outcomes. However, there is limited research examining health care utilization patterns among patients identified with high social isolation. We evaluated the associations between social isolation and hospital and emergency department (ED) utilization among Medicare patients $65+$ years of age.

Methods: We analyzed a retrospective cohort of 18,557 Medicare patients age 65+ who completed a Medicare Total Health Assessment survey during January 1, 2016-June 30, 2018. The Total Health Assessment is an operations-based survey of quality of care among Medicare members at Kaiser Permanente Northwest, an integrated health care system in Oregon and southwest Washington. Social isolation was assessed by asking patients how often (5-point Likert scale: always, often, sometimes, rarely, never) they felt lonely or social isolated from those around them. Hospital and ED utilization, demographic, and clinical data were obtained via electronic health record. The primary outcomes included any hospital admission (binary) and any ED visit (binary) in the 12 months following index survey date. Multivariable logistic regression models were used to assess the association of social isolation (often/always, sometimes vs rarely/never [reference group]) with hospital and ED utilization, adjusting for age, sex, area deprivation index, Charlson comorbidity index (CCI), any hospital admissions, or ED utilization prior to the index survey date.

Results: Patients' average age was 73.4 years; $56.5 \%$ were female, and $25.2 \%$ had a CCI score of $\geq 2$. Those who reported social isolation "sometimes" were more likely to have any hospital admissions (odds ratio [OR]: 1.17, 95\% CI: $1.01-1.35, \mathrm{P}=0.04$ ), compared to those who "rarely/ never" experienced social isolation. In adjusted models, those that experienced social isolation "sometimes" or "always/often" were more likely to have any ED visits (OR: 1.28, 95\% CI: 1.15-1.41, P<0.0001, and OR: 1.51, 95\% CI: $1.25-1.84, \mathrm{P}<0.0001$, respectively) compared to those who "rarely/never" experienced social isolation.

Conclusion: These findings suggest that self-reported social isolation may be predictive of future hospital admissions and ED utilization, reflecting the importance of assessing and documenting social isolation among patients. It is imperative to better understand how addressing social isolation needs within the health care system may reduce hospital admission and ED utilization, and ultimately improve health outcomes.

P9.02

Variation in Quality Metrics Across Adult Asian Ethnic Groups: The Kaiser Permanente Northern California DECKA2016 Cohort Study

Nancy P. Gordon, ${ }^{1}$ Joan C. Lo ${ }^{1}$

\section{${ }^{1}$ Kaiser Permanente Northern California, Oakland, CA}

Background: A recently published study based on electronic health record data for a Kaiser Permanente Northern California adult cohort (DECKA2016) showed significant variation in prevalence of chronic conditions and risk factors across Asian ethnic groups and between Asian ethnic groups and an aggregated Asian race group. This study used the same cohort to examine whether similar ethnic variation would be seen in Healthcare Effectiveness Data and Information Set (HEDIS ${ }^{\circledR}$ ) quality metrics.

Methods: Algorithms used to identify HEDIS-eligible cohorts for cervical, breast, and colorectal cancer screening, blood pressure management (BPM), and diabetes management (A1c test, A1c of $<8, A 1 \mathrm{c}$ of $>9$, DiabBPM) were applied to the DECKA2016 cohort. Age-sex and age-standardized (to 2016 U.S. Census) quality metrics were produced for All Asians, Filipinos, Chinese, Japanese, Vietnamese, South Asians, and Native Hawaiian/Pacific Islanders (PIs).

Results: Metrics for Asians were $90 \%$ for cervical cancer screening, $89.3 \%$ for breast cancer screening, $85.3 \%$ for colorectal cancer screening, $67.7 \%$ for A1c of $<8,21.3 \%$ for A1c of $>9$, and $89.3 \%$ for DiabBPM. Compared to Asian race statistics, Asian ethnic groups did not significantly differ 
on cervical or breast cancer screening, but Filipinos, South Asians, and PIs were lower and Chinese and Vietnamese higher on colorectal cancer screening. Among hypertensives, Chinese were higher and PIs lower on BPM, but other ethnic groups were similar to the Asian metric. Among diabetics, ethnic groups were similar for A1c testing, but Filipinos and PIs were lower on A1c of $<8$ and higher on A1c of $>9$, while the opposite was true for Chinese and South Asians. Filipino and PI diabetics also were lower on the BPM metric. PIs were very similar to Blacks on the BPM and diabetes management metrics, while Filipinos did better than Blacks on BPM, A1c of $<8$, and A1c of $>9$.

Conclusion: In our earlier study, we found wide variation across Asian ethnic groups in prevalence of cardiovascular risks, with Filipinos and PIs having similar prevalence profiles to Blacks and Latinos. In this study, we found much narrower variation in quality metrics across Asian ethnic groups, although quality metrics for Filipinos and PIs tended to be worse than for Chinese and South Asians. Further research is needed to identify reasons why Filipinos and PIs are at higher risk of chronic cardiovascular conditions and are having more difficulty managing these conditions.

\section{P9.03}

\section{People in Communities: Can We Use Community Social Need Indicators as Predictors for Individual Social Need?}

Morgan J. Fuoco, ${ }^{1}$ Michael Leo, ${ }^{1}$ Lindsay Jenkins, ${ }^{1}$ Mary Ann McBurnie, ${ }^{1}$ Stephanie L. Fitzpatrick ${ }^{1}$

\section{${ }^{1}$ Kaiser Permanente Northwest, Portland, OR}

Background: Social determinants of health, including the economic, social, and environmental characteristics of communities where people live, affect a wide range of health outcomes and risks. Research suggests that social needs contribute more to health outcomes than clinical factors. Currently, community-level data can be collected more efficiently and cost-effectively, while collection of patientlevel data requires additional resources.

Methods: We sought to understand the relationship between tract-level social need measures and individual-level social need screener responses. For the individual-level measures, we used data from the Your Current Life Situation (YCLS) survey, a social needs screener administered at Kaiser Permanente. For the community measures, we used data from the American Community Survey and indices from the Robert Graham Center. Our sample $(\mathrm{N}=6457)$ consisted of adults, 18 years of age or older, who were administered the YCLS during 2017. First, we assessed the degree to which individual responses to the YCLS and community-level variables were correlated. Next, we analyzed odds ratios from a mixedeffects logistic regression model using the community-level variables as predictors of individual responses to the YCLS.

Results: The largest standardized effect size was 0.20 between social need (yes or no) and the Social Deprivation Index (SDI). Most correlations, however, were between -0.03 and 0.05 , suggesting that there is a low level of correlation between the two levels of measurement for a given set of items. In the multivariable model, many of the associations were significant, with odds ratios ranging from 1.14 to 1.25 , particularly among specific needs and living below $100 \%$ of the federal poverty level. For every 1-point increase in the Gini Index, there was a $2 \%(95 \%$ CI: $1.01-1.02)$ increase in the odds of reporting at least 1 financial insecurity. For every 1-point increase in SDI score, the odds of reporting any social need increased by $2 \%$ (95\% CI: $1.01-1.02)$.

Conclusion: The bivariate results suggest that most tractlevel community variables are poor proxies of individuallevel social needs. However, there is some ability of federal poverty indicators at the community level to predict individual financial needs, suggesting some community metrics may be useful in characterizing patient individual financial insecurity.

\section{P9.04 \\ Role of External Incentives in Initiating Electronic Health Record-Based Social Determinants of Health Data Collection in Community Health Centers}

Inga Gruß,1 Arwen Bunce, ${ }^{2}$ James Davis, ${ }^{1}$ Katie Dambrun, ${ }^{2}$ Erika Cottrell, ${ }^{2}$ Rachel Gold ${ }^{1}$

${ }^{1}$ Kaiser Permanente Center for Health Research, Portland, OR; ${ }^{2} \mathrm{OCHIN}$, Inc., Portland, OR

Background: Successfully incorporating social determinants of health (SDH) screening into clinic workflows can help care teams provide targeted care and appropriate referrals or other interventions for patients reporting SDH needs. However, integrating SDH screening into clinical routines is notoriously challenging. To achieve broad implementation of SDH screening, an improved understanding of the factors that can facilitate effective implementation is necessary.

Methods: We interviewed 43 health care professionals at 8 safety-net community health center (CHC) organizations across the United States that had adopted an electronic health record (EHR)-based SDH documentation tool with no outside implementation support. Interviewees included staff in administration, quality improvement, informatics, front desk, clinical (providers, nurses, behavioral health), and community health worker roles. Interviews focused on why and how each organization integrated these tools into clinic workflows and on factors that affected the adoption of this practice change.

Results: External motivators were one important factor that facilitated effective introduction and integration of EHRbased SDH screening. External motivators that supported the introduction of SDH screening included grant requirements and participation in accountable care organizations or certification processes. For example, through participation in accountable care organizations, integration was facilitated as CHCs were reimbursed for the needed additional care steps 
or were able to make additional hires to support SDH data collection.

Conclusion: Identifying and addressing factors that can support the successful integration of SDH screening into clinic workflows is necessary to support widespread SDH screening implementation. Creating and maintaining a variety of external motivators may be an effective tool to help CHCs and others initiate and sustain SDH screening.

\section{P9.05}

Advantages, Limitations, and Key Themes for Integrating Community Health Workers in a Large Health Care Delivery System for Diabetes Management Among Vulnerable Populations: Qualitative Findings

\section{Dea Papajorgji-Taylor, ${ }^{1}$ Jennifer $\quad$ L. $\quad$ Schneider, ${ }^{1}$} Stephanie L. Fitzpatrick ${ }^{1}$

\section{${ }^{1}$ Kaiser Permanente Northwest, Portland, OR}

Background: There is increasing evidence that traditional health workers can play an essential role in chronic disease management and helping individuals address unmet basic needs. The Bridge to Health study, an ongoing 2-year clinical trial, is exploring whether utilizing community health workers (CHWs) to navigate patients from underserved populations (racial/ethnic minorities and patients on Medicaid) to resources and provide evidence-based diabetes self-management training may be more effective than navigation alone in an integrated health care delivery system. To begin to assess determinants of implementation of a CHW-led intervention in an integrated health system, we conducted qualitative interviews with senior health system leaders, health system navigators, and CHWs.

Methods: In-depth semi-structured guides were developed, and interviews were conducted by phone and in person. Interviews were audio-recorded, transcribed, and coded using NVIVO software. Thematic analysis identified key themes related to advantages, limitations, and recommendations for integrating $\mathrm{CHW}$ in the health care system to support patients with unmanaged diabetes. Seven interviews were completed with senior health system leaders, and a group interview was conducted with $6 \mathrm{CHWs}$. (The group interview with the health system navigators is being scheduled and results will be available to incorporate in the final presentation).

Results: Initial interview findings demonstrate the value and importance seen by health system leaders in collaborating with community partners to extend reach in providing culturally sensitive care and navigation services to diverse patient populations. Clearly defined roles and expectations for the partnership to be effective between a $\mathrm{CHW}$ and the clinical care team was a reoccurring theme that came up for both health system leaders and the CHWs. The advantages that CHWs can bring to the patient's overall clinical care, based on their positioning in the community and associations with various community-based organizations, were recognized as valuable.

Conclusion: The qualitative findings for this study will offer guidance to and serve as a critical step in understanding how to best integrate CHWs in a health care delivery system for providing comprehensive care and resource navigation toward eliminating health disparities in diabetes management.

P9.06

\section{Financial Difficulties in Families With Children With a Chronic Health Condition}

\author{
Alexandra Varga, ${ }^{1}$ Joanna Bulkley, ${ }^{1}$ John Dickerson, ${ }^{1}$ \\ Beth Waitzfelder, ${ }^{1}$ Lisa Croen, ${ }^{2}$ Eric Fombonne, ${ }^{3}$ \\ Phillip Crawford, ${ }^{1}$ Frances Lynch ${ }^{1}$
}

\section{${ }^{1}$ Kaiser Permanente Northwest, Portland, OR; ${ }^{2}$ Kaiser Permanente Northern California, Oakland, CA; ${ }^{3}$ Oregon Health \& Science University, Portland, OR}

Background: It has been well documented that children with chronic health conditions may incur higher costs than children without these conditions. Some of these costs may not be covered by insurance and may lead to financial difficulties. More than $15 \%$ of children suffer from a chronic health condition, yet little is known about the prevalence of financial strain in these families and whether it varies by chronic condition.

Methods: Using electronic health records from 3 Kaiser Permanente sites and several safety-net clinics, we identified 3 groups of children (3-16.5 years of age): 1) those with autism spectrum disorders (ASD); 2) those with asthma (Asthma); and 3) those without these conditions (GenPop). Groups were matched on age and gender. Eligible families that consented to participate completed an online survey about costs, insurance, income, financial difficulties, education, and employment. Among those participants who answered at least 1 financial question, we report demographic characteristics of the children and caregivers (the respondent), summarize responses about financial difficulties, and compare the groups using chi-squared and ANOVA tests.

Results: Of 1461 families in the study, 1320 responded to 1 or more financial question. Age and gender were balanced between groups; overall, $79.1 \%$ were male and average age was 9.15 . About $44.6 \%$ of GenPop, $37.0 \%$ of ASD, and $37.5 \%$ of Asthma families reported a monthly income of more than $\$ 6000$. Employment status was significantly different between groups $(\mathrm{P}<0.001) ; 26.6 \%$ of the ASD respondents were not employed compared to $19.9 \%$ for Asthma and $19.7 \%$ for GenPop. Also, $54.1 \%$ of families of children with ASD reported financial difficulty related to the child's health compared to $28.8 \%$ for Asthma and $16.7 \%$ for GenPop $(\mathrm{P}<0.001)$. Similarly, $5.9 \%$ of families of children with ASD borrowed money to pay for medical services compared to $2.6 \%$ for Asthma and $1.7 \%$ for GenPop $(\mathrm{P}=0.002)$. In the ASD group, $60 \%$ of respondents reported distress due to the cost of 
their child's medical care compared to $32.5 \%$ of Asthma and $20.9 \%$ of GenPop.

Conclusion: Findings provide evidence of higher financial burden for families with children with chronic health conditions compared to those with no significant health conditions. This is particularly pronounced in families of children with ASD.

\section{P9.07}

\section{Homelessness Within and Beyond the Hospital Walls: Exploring the Documentation and Use of Diagnosis Codes to Identify Homelessness}

\section{Navin Vij, ${ }^{1}$ Keshab Subedi, ${ }^{1}$ Adebayo Gbadebo ${ }^{1}$ \\ ${ }^{1}$ ChristianaCare, Wilmington, DE}

Background: It is widely acknowledged that health outcomes are determined not only by access to care but also by the social and economic conditions that shape an individual's daily life - the social determinants of health, including homelessness. Individuals who experience homelessness have higher rates of untreated chronic physical and behavioral health conditions, which contribute to higher rates of health care utilization and increased mortality compared to the general population. In recent years, health systems have begun investing resources in identifying such patients. One emerging method to help quantify the impact of homelessness is the use of electronic medical record data, including International Classification of Diseases (ICD) codes to identify clinical encounters. With the shift to ICD10 , systems have an opportunity to understand how the expanded use of $Z$ codes can help identify and intervene on the social factors influencing the health of patients.

Methods: We conducted a retrospective, time- and propensity score-matched cohort design to identify individuals with homelessness using ICD-10 code for Z59, a residential address listed as a shelter or none, or a social work consultation for homelessness with an emergency room visit or hospitalization from October 2015 to September 2019 at ChristianaCare.

Results: More than 2600 unique patients were identified as homeless through encounters with the emergency room and hospitalizations as inpatients. A summative evaluation of this population's visits will assess outcomes, including hospital readmissions, length of stay, discharge disposition, primary and secondary reasons for presentation, and health-related medical comorbidity including associated behavioral and medical conditions. These will be compared to a matched cohort balanced with respect to age, gender, race, insurance status, comorbidities, and rate of hospitalization prior to the index visit, with a particular focus on disparities in care and outcomes. Furthermore, we will compare the use of ICD10 diagnosis codes to the other two methods of identifying homelessness, including rates of overlap.

Conclusion: Three methods of identifying homelessness yielded different estimates, suggesting that further research into the validity of diagnosis codes to assess homelessness is needed. Increasing awareness of their application could provide timely information for health systems to improve interventions for patients experiencing homelessness.

\section{P9.08 \\ Disparities in Opioid Prescribing Rates by Dentists Based on Patient Gender, Race/Ethnicity, and Sociodemographic Status}

\author{
D. Brad Rindal, ${ }^{1}$ Steve E. Asche,${ }^{1}$ Sheryl Kane,${ }^{1}$ Anjali \\ R. Truitt, ${ }^{1}$ Lauryn Davin, ${ }^{1}$ Tracy Shea, ${ }^{1}$ Donald Worley, ${ }^{1}$ \\ Jan Gryczynski, ${ }^{2}$ Shannon G. Mitchell ${ }^{2}$
}

${ }^{1}$ HealthPartners, Bloomington, MN; ${ }^{2}$ Friends Research Institute, Inc., Baltimore, MD

Background: Data examining disparities in opioid prescribing patterns related to oral pain management come largely from emergency rooms, where patients present with dental emergencies. Reports examining disparities in prescribing patterns in ambulatory dental settings are lacking.

Methods: This cross-sectional study examined disparities in opioid prescribing among patients undergoing dental procedures from September 1, 2018, to August 31, 2019, representing a 12-month baseline period for a clinical trial focused on de-implementing opioid prescribing for dental extraction procedures. The sample consists of 175,091 encounters (165,316 nonextraction + 9775 extraction) from 90,215 patients of all ages undergoing any dental procedure (except exclusively preventive or orthodontic procedures) conducted at 22 dental clinics by 66 dental providers. All data come from the electronic health record. The encounter-level endpoint is a binary variable indicating that the patient was prescribed an opioid analgesic within 7 days of their dental procedure.

Results: More than half of patients at encounters were female (57\%); 22\% were under age 18 years, 32\% were $18-45,27 \%$ were $46-65$, and $19 \%$ were 66 or older; $60 \%$ were white; and $34 \%$ had public-pay insurance. Opioid prescribing within 7 days occurred at $1.9 \%$ of encounters and varied by procedure: $25.8 \%$ at extraction encounters, and $0.5 \%$ at nonextraction encounters. Among nonextraction encounters, opioid prescribing was most likely for nonextraction oral surgery $(17.3 \%)$ and prosthodontic fixed (8.0\%) procedures. Opioid prescribing rates were highest for encounters with patients 18-25 years of age (6.7\%); Pacific Islander (4.2\%), Native American $(2.9 \%)$, or Black $(2.7 \%)$ race; and those with public-pay insurance (2.8\%). Opioid prescribing was highest for encounters with an oral surgeon $(33.2 \%)$, followed by periodontist $(0.7 \%)$, general dentist $(0.7 \%)$, and endodontist $(0.6 \%)$.

Conclusion: Opioid prescriptions are most common for extractions and other nonextraction oral surgery procedures. Higher rates of prescribing occur in younger age groups, minority populations, and lower socioeconomic groups. This 
inequity could be partially explained by more oral disease and subsequent need for extractions in these groups.

\section{P9.09}

\section{Using Patient Activation Measure and Decisional Conflict Scale to Assess the Relationship Between Levels of Self-Management and Decisional Conflict in Southeastern Patients With Chronic Kidney Disease}

\section{Serena Kongara, ${ }^{1}$ Musu Sesay, ${ }^{2}$ Doraina Walker- Williams, ${ }^{2}$ Lee Cromwell, ${ }^{2}$ Nirvan Mukerji, ${ }^{2}$ Jennifer Gander $^{2}$ \\ ${ }^{1}$ Tulane University, New Orleans, LA; ${ }^{2}$ Kaiser Permanente Georgia, Atlanta, GA}

Background: Self-management has been shown to be beneficial in reducing the progression of kidney loss in patients with chronic kidney disease (CKD). High levels of patient activation are correlated with high levels of selfcare among patients with CKD. We aimed to use the patient activation measure and decisional conflict scale to discover barriers to care.

Methods: Focus group participants diagnosed with CKD $(\mathrm{n}=28)$ completed 2 surveys following the focus group discussion. Each patient answered a 13-item patient activation measure survey to measure their level of activation and a 16item decisional conflict survey measured on a scale from 0 (no decisional conflict) to 100 (extremely high decisional conflict). Patient activation was categorized into 4 levels: disengaged and overwhelmed; becoming aware but still struggling; taking action and gaining control; and maintaining behaviors and pushing further. Kruskal-Wallis and chi-squared tests compared decisional conflict and patient activation across demographics, respectively, and Pearson correlation determined the correlation between decisional conflict and patient activation.

Results: Patients with CKD who completed the surveys $(\mathrm{n}=28)$ were $67 \%$ male, $82 \%$ black, and had a mean age of 54.8 years (standard deviation: 8.3). Median decisional conflict score was 17.2 (interquartile range [IQR]: 0-27.3). Females had higher decisional conflict (median: 23.4, IQR: 17.2-34.4) compared to males (median: 11.7, IQR: 0-23.4; $\mathrm{P}=0.1094$ ). Based on the Patient Activation Measure, 4 patients (15.4\%) were categorized as disengaged and overwhelmed or becoming aware but still struggling. No significant difference in patient activation was found for gender $(\mathrm{P}=0.491)$ or race $(\mathrm{P}=0.798)$. Decisional conflict and patient activation were significantly inversely correlated $(\mathrm{R}=-0.789, \mathrm{P}<0.0001)$.

Conclusion: Patients with CKD experience various barriers to managing their disease. High patient activation was inversely correlated with low decisional conflict. Patients with CKD may be conflicted or feel overwhelmed when determining the best course of action for their treatment or be struggling with their CKD diagnosis. Female patients may have a harder time making a decision. Health care professionals should consider the gender differences in decisional challenges and counsel all their patients to help them make decisions and become activated in their disease management.

P9.10

Systematic Availability of Education Data in Electronic Health Records Could Be Valuable for Clinical Care, Population Health Management, and Research

\author{
Nancy P. Gordon, ${ }^{1}$ Joan C. Lo ${ }^{1}$ \\ ${ }^{1}$ Kaiser Permanente Northern California, Oakland, CA
}

Background: Educational attainment is recognized as an important social determinant of health. Research indicates that better-educated adults have a healthier lifestyle, have higher health literacy, are at lower risk for developing chronic conditions, and are more likely to use online health resources. While paper medical charts usually contained patient education information, education is often not available in electronic health records (EHR), thus making it unavailable for clinical care, population health management, and research. This study examined the relationship of education with EHRderived health-related characteristics in a Medicare-age cohort.

Methods: Using survey, clinical questionnaire, and EHRentered data, we were able to assign approximately $49 \%$ $(n=135,503)$ of white, $46 \%(n=13,652)$ of black, and $41 \%$ $(n=13,239)$ of English-speaking Latino members of Kaiser Permanente Northern California (KPNC), 65-79 years of age, in the KPNC DECKA2016 study cohort to an education category (did not graduate high school $[<\mathrm{HSG}]$, high school graduate $[\mathrm{HSG}]$, some college $[\mathrm{SC}]$, or college graduate $[\mathrm{CG}])$. We then used EHR-derived data to produce and compare age, sex, and age-standardized prevalence of health risks (current and ever smoking, body mass index [BMI] of $\geq 30$, body mass index [BMI] of $\geq 35$ ), diagnosed chronic conditions (diabetes, hypertension, coronary artery disease, heart failure, chronic obstructive pulmonary disease), diabetes and hypertension control quality metrics, and patient portal use (having an activated www.kp.org patient portal account, sending 1 or more secure message, and viewing 1 or more online lab result) by level of education for white, black, and Latino women and men.

Results: Compared with $<\mathrm{HSG}$, HSG, and, in many instances, SC adults, CGs in all race/ethnic groups (overall and by sex), had lower prevalence of smoking (current and ever), obesity (BMI of $\geq 30$, BMI of $\geq 35$ ), and all 5 chronic conditions. Having an activated patient portal account and using patient portal features increased as level of education increased. Educational attainment was not associated with diabetes or hypertension control quality metrics. Race/ethnic and sex differences were frequently observed within the same level of education. 
Conclusion: Educational attainment is associated with disparities in health risks, health conditions, and patient portal use within and across racial/ethnic groups. Promoting systematic capture of education as a categorical EHR data element could help improve clinical care delivery, population health management, and research.

\section{DATA SCIENCE, INFORMATICS, AND COMMON DATA MODELS}

\section{OA9.01 \\ Putting the Virtual Into the Virtual Data Warehouse: Developing New Encounter Types for Virtual Care}

\author{
Heather Tavel, ${ }^{1}$ Daniel $\mathrm{Ng}^{2}$ \\ ${ }^{1}$ Kaiser Permanente Colorado, Denver, CO; ${ }^{2}$ Kaiser \\ Permanente Northern California, Oakland, CA
}

Background: The delivery of health care is changing rapidly, with increasing use of "virtual" care encounters using different modes of technology-mediated interaction between patients and providers, for example, video visits, online chats, and e-visits. Updating the HCSRN virtual data warehouse (VDW) data model to accurately represent these new types of encounters is vital to HCSRN research. The VDW Utilization Workgroup is exploring the available data within HCSRN sites to inform the creation of new encounter types and subtypes to enable research on these emerging methods of delivering care.

Methods: An exploratory program was written and distributed to HCSRN sites to collect and summarize electronic medical record (EMR) encounter data, from 2015 through 2018, that may be potentially classified as virtual care. Text string searches were used to capture a wide range of potential encounter types. Encounter types falling outside the text search were manually reviewed to capture additional potential encounter types. Individual sites were asked to investigate ambiguous encounter types. Methods of identifying virtual care from billing data also were examined. The results were compiled to demonstrate similarities and differences in how virtual care is represented in EMR source systems throughout the research network.

Results: Eleven HCSRN sites returned results. All 11 sites had encounter types for telephone visits and emails; 9 sites had e-visits and video visits. Only 2 sites had an encounter type for online chat with a provider. There was a lot of variation in source systems' descriptions of encounter types. Volumes of virtual care have been growing steadily at all sites since 2015 . Conclusion: Virtual care encounter types are increasingly becoming standard practice among HCSRN sites. Within our current VDW data model, more granularity may be needed to support research involving the various modes of virtual care. In addition, our definitions of what constitutes an "encounter" may require changes to tease out specific types of virtual care, for example, telemedicine. Further analysis and discussion are needed to understand how best to represent these care methods to support research needs in our rapidly evolving landscape of health care delivery.

\section{OA9.02 \\ Early Detection of Hospital-Acquired Acute Kidney Injury From Electronic Health Record Data Using Machine Learning}

Yirui Hu, ${ }^{1}$ Gurmukteshwar Singh, ${ }^{1}$ Kunpeng Liu, ${ }^{2}$ Sara Saunders, ${ }^{1}$ Dave Riviello, ${ }^{1}$ Jason Brown, ${ }^{1}$ Kevin Ho, ${ }^{3}$ H. Lester Kirchner ${ }^{1}$

${ }^{1}$ Geisinger Health, Danville, PA; ${ }^{2}$ University of Central Florida, Orlando, FL; ${ }^{3}$ Sanofi Genzyme, Cambridge, MA

Background: Hospital-acquired acute kidney injury (HA$\mathrm{AKI}$ ) is a common syndrome associated with increased risk of morbidity and mortality. Early prediction of HA-AKI using electronic health record data is desirable, as it enables clinicians to match appropriate treatment to minimize risk and severity.

Methods: All inpatient admissions from July, 13, 2012, to July 11, 2018, who had serum creatinine measured at Geisinger Health were included. Patients with end-stage renal disease, length of stay of $<48$ hours, and acute kidney injury on admission were excluded. A validated algorithm was used to determine baseline renal function. Acute kidney injury was defined according to KDIGO guidelines. Machine learning algorithms were implemented to predict encounters where the patient developed HA-AKI beyond the initial 24 hours of admission. Fifty input variables, including demographics, initial laboratory values taken within the first 24-hours of admission, active medications at time of admission, and prevalent comorbidities, were entered into machine learning algorithms (random forest, XGBoost, logistic regression). Multiple imputation by chained equations was implemented for missing variables. Univariate feature selection was implemented wherein variables were ranked by evaluating contribution to classification outcome. Randomized search strategy was performed to obtain the optimal hyperparameter set for each algorithm. Models were evaluated using a mean area under the receiver operating characteristic curve (AUC) over 5 -fold cross-validation.

Results: Among 209,300 inpatient admissions, 26,410 $(12.6 \%)$ developed HA-AKI. For acute kidney injury prediction, the AUC of the full model was 0.88 for both random forest and XGBoost and 0.86 for logistic regression. To balance the trade-off between model simplicity and performance, 23 variables from univariate feature selection evaluated using random forest were selected in predicting HA-AKI (AUC: 0.87 ). The probability cut-off point of acute kidney injury prediction outcome was determined using Youden's index based on the balance between false positives 
and false negatives. A probability cutoff of $>0.23$ provided sensitivity and specificity of 0.78 and 0.81 , respectively.

Conclusion: Our machine learning algorithm applied at 24 hours of admission identifies patients at risk for HA-AKI with high accuracy. Significant variables included in this algorithm should be monitored in real time to allow early identification and preventive interventions in patients at risk for HA-AKI.

\section{OA9.03 \\ Recalibration and Additional Data Domains Lead to Modestly Improved Performance of Risk Calculators for Heart Failure Readmission}

\author{
Samuel Savitz,, Keane Lee, ${ }^{1}$ Jamal Rana, ${ }^{1}$ Thomas \\ Leong, ${ }^{1}$ Sue Hee Sung, ${ }^{1}$ Grace Tabada, ${ }^{1}$ Alan Go ${ }^{1}$
}

\section{${ }^{1}$ Kaiser Permanente Northern California, Oakland, CA}

Background: Heart failure (HF)-related hospitalizations are a growing public health burden, especially among older adults. We evaluated the performance of several published risk calculators for predicting 30-day readmission after heart failure hospitalizations: 1) using the original coefficients for the existing risk calculators; 2) updating the coefficients based on data from a new, ethnically diverse HF population; and 3) developing a new model that incorporates additional variables in addition to updated coefficients for the original variables. We hypothesized that recalibrating model coefficients for the new HF population and incorporating additional variables would improve the performance of these existing 30-day readmission risk calculators.

Methods: We identified 45,059 adults hospitalized for HF during 2012-2017 within Kaiser Permanente Northern California, an integrated health care delivery system. We used an $80 \% / 20 \%$ split sampling for development and validation testing. The risk calculators tested included LACE index, LACE+ Index, and Yale CORE. For the model with additional variables, we included all variables used in the original models plus additional variables, including cardiovascular medication use and socioeconomic status. We used gradient boosting with $k$-fold cross-validation to avoid overfitting. We assessed model performance using area under the curve (AUC) and calibration plots.

Results: Discrimination (AUC) was poor using original models: LACE (0.58 [95\% CI: 0.57-0.60]), LACE+ $(0.56$ [95\% CI: 0.54-0.58]), and Yale CORE (0.55 [95\% CI: $0.54-0.57]$ ). Recalibrating variable coefficients in these models resulted in small improvements in discrimination for LACE+ $(0.58$ [95\% CI: 0.57-0.60]) and Yale CORE (0.58 [95\% CI: $0.57-0.60]$ ), but not for LACE. Including the additional variables also resulted in a small improvement in AUC for the model using gradient boosting (0.61 [95\% CI: 0.59-0.62]). Calibration plots showed generally good calibration across all models except for the Yale CORE model that used the original variable coefficients.

Conclusion: Recalibrating variable coefficients and incorporating prior medication and socioeconomic status variables led to small, statistically significant improvements in model discrimination while maintaining good calibration for published models to predict readmission. However, overall performance improvements are needed to increase the utility of these published risk calculators for readmission after discharge from a HF hospitalization.

\section{OA9.04 \\ Building Medical Device Identifiers Into Longitudinal Data: The BUILD Distributed Data Network}

Jove Graham, ${ }^{1}$ Joseph Muhlestein, ${ }^{2}$ James Tcheng, ${ }^{3}$ Andrew McKinnon, ${ }^{4}$ Stacey Knight, ${ }^{2}$ Heidi May, ${ }^{2}$ Tami Bair, ${ }^{2}$ Kevin Capatch, ${ }^{1}$ Joseph Drozda ${ }^{4}$

${ }^{1}$ Geisinger Health, Danville, PA; ${ }^{2}$ Intermountain Healthcare, Salt Lake City, UT; ${ }^{3}$ Duke University, Durham, NC; ${ }^{4}$ Mercy Health System, Chesterfield, MO

Background: Drug eluting stents are a standard of care for percutaneous coronary intervention (PCI), but limited data exist to guide drug-eluting stent choice. In 2014, the U.S. Food and Drug Administration began requiring manufacturers to label medical devices with unique device identifiers (UDIs), similar to National Drug Code numbers for drugs, which potentially enables large-scale observational studies of medical devices that were not previously possible. Three large health systems - Mercy, Geisinger, and Intermountain collaborated to create the Building UDI into Longitudinal Data (BUILD) network to provide real-world evidence on medical devices. Modeled on other distributed data networks such as the HCSRN virtual data warehouse, BUILD combines device, registry, and other patient data via a common data model and allows common queries to be programmed and distributed to all participating sites without sharing of identifiable patient data among systems. The purpose of this study was to perform an outcomes analysis across the network, comparing 1-year outcomes for patients with two types of drug-eluting stents.

Methods: We performed a retrospective analysis of patients receiving zotarolimus- or everolimus-eluting stents from 2012 to 2019. The distributed analysis examined 32 baseline covariates, created propensity-matched cohorts at each site, assessed these cohorts for confounding, and compared 1-year events such as subsequent PCI procedures, death, and a composite endpoint including acute myocardial infarction, stroke, reoperation, or death. Cox proportional hazards (ie, survival) analysis was used, with SAS 9.4 software and P-values less than 0.05 considered statistically significant.

Results: We identified 9,045 patients (4859 Mercy, 4059 Geisinger, 127 Intermountain; mean age: $65 \pm 12$ years; sex: $69 \%$ male; stent: $23 \%$ zotarolimus). Estimates of hazard ratios (zotarolimus vs everolimus) at the 3 sites ranged from 0.85 to 4.86 for subsequent PCI (P ranged from 0.10 to 0.35 ), 1.06 to 2.12 for death (P ranged from 0.16 to 0.78 ) and 0.94 to 3.02 for the composite endpoint (P ranged from 0.16 to 0.53 ). In 
summary, there were no significant differences in the hazards of these outcomes, consistent with what was suggested by the early clinical trial data for these drug-eluting devices.

Conclusion: This large real-world, multicenter study showed no statistical differences in 1-year outcomes of patients receiving zotoralimus and everolimus stents - results similar to other randomized controlled studies. This demonstration project shows that multi-institutional data networks can provide and advance clinically relevant real-world evidence.

\section{P5.01}

\section{Performance Characteristics of a Computable Phenotype Algorithm to Identify Patients With Hematuria in Electronic Health Records}

\section{Tullika Garg, ${ }^{1}$ Mudit Gupta, ${ }^{1}$ Matthew Nielsen, ${ }^{2}$ H. Lester Kirchner ${ }^{1}$}

${ }^{1}$ Geisinger Health, Danville, PA; ${ }^{2}$ University of North Carolina at Chapel Hill, Chapel Hill, NC

Background: Two million patients per year are referred to urologists for hematuria (blood in the urine). Current American Urological Association hematuria evaluation guidelines recommend multiphase computed tomography (CT) and flexible cystoscopy. However, multiphase CT is associated with a high risk of secondary malignancies from imaging-related radiation exposure. To better understand population-based patterns of CT imaging associated with hematuria evaluations, we sought to develop a computable phenotype to accurately identify hematuria cases from electronic health records.

Methods: We developed rules to identify patients with hematuria and to differentiate between gross and microscopic hematuria using a combination of ICD-9 and ICD-10 diagnosis codes, urine color values, and urine red blood cell microscopy values. We used an iterative process to refine the algorithm on a gold-standard, chart-reviewed cohort of 3095 hematuria cases. We then applied the algorithm to all Geisinger Health patients who were 35 years or older and had at least 3 completed visits within a 3 -year window. We conducted a chart review to determine performance characteristics of the computable phenotype algorithm.

Results: We identified 767,209 patients who met inclusion criteria. After applying the computable phenotype, we identified 37,332 hematuria cases, 511,177 controls, and 218,700 indeterminate cases. Of the hematuria cases, 11,924 were categorized as gross, 24,555 as microscopic, and 853 as uncategorized due to diagnosis codes for both gross and microscopic hematuria. Indeterminate had a signal in the form of a single diagnosis code, urine color, or urine microscopy value, but without adequate additional information to categorize. The positive predictive value (PPV) of identifying hematuria cases was $100 \%$, and the negative predictive value (NPV) was 99\%. The gross hematuria algorithm had a PPV of $100 \%$ and NPV of $99 \%$. The microscopic hematuria algorithm had a lower PPV of 78\% and NPV of 100\%.

Conclusion: Computable phenotypes facilitate standardized data queries across electronic health records. We developed an algorithm utilizing diagnosis codes and urine lab values that accurately identifies hematuria cases and categorizes each into gross or microscopic hematuria. This algorithm will enable researchers and clinicians to identify hematuria cases and determine downstream patterns of care and outcomes for this common condition.

\section{P5.02}

\section{Privacy Preserving Fuzzy Patient Matching Using Homomorphic Encryption}

\section{Shiva Ashish Thumparthy, ${ }^{1}$ llya Sher ${ }^{2}$}

${ }^{1}$ Brainlab AG, Munich, Germany; ${ }^{2}$ Beame.io, Tel Aviv, Israel

Background: Medical record interoperability has been a focus of health care systems for purposes of providing better patient outcomes. However, in an age of increasing privacy and confidentiality concerns, comparison of unencrypted patient demographics is obsolete due to strict guidelines mandating protection of sensitive patient information. In addition, while dealing with data from multiple sources, demographics are subject to errors (eg, character transpositions) and changes (eg, address relocations, lastname changes. Therefore, an important step in realizing this interoperability goal is identifying patients with records across facilities through record linkage algorithms, in a privacy-preserving fashion that is resilient to data entry inconsistencies, ie, in a fuzzy and probabilistic manner.

Methods: A common building block to encode patient attributes is a Bloom filter. Bloom filters represent a digest of information without containing any original information in plain text. However, Bloom filters were subject to scrutiny due to brute force and frequency attacks. Homomorphic encryption is a form of encryption that allows computation on encrypted data. The result of the computation, when decrypted, is the same as if the operations had been performed on unencrypted data. Homomorphic encryption of Bloom filters and subsequent comparison is, therefore, resilient to these attacks. Our primary contribution is a method of matching a list of encrypted patients at once as opposed to pairwise comparisons of encrypted Bloom filters. This significantly reduces computational complexity and communication overheads. We achieve this using homomorphic encryption ciphertext packing techniques, which allow for encrypting large volumes of data into a single ciphertext. This allows for computations on entire matrices of data as opposed to individual row vectors.

Results: The optimized matching algorithm yields a conservative preliminary speedup of 7 times during encryption and 266 times during matching by multiplication on $16 * 16$ matrices, while generating 1 ciphertext per matrix as opposed 
to the previous state-of-the-art of 1 ciphertext per row vector. Conclusion: We take real-world limitations into consideration while designing a privacy-preserving patient matching solution using homomorphic encryption that significantly lowers computational costs and communication overheads. Future findings will include statistics for larger matrices.

\section{P5.03}

\section{Changes in Population Demographics From Expanding the Virtual Data Warehouse to Multiple Sites in a Large Integrated Health Care Network}

\author{
Satish Mudiganti, ${ }^{1}$ Pragati Kenkare, ${ }^{1}$ Kevin Hays, ${ }^{1}$ \\ Robert Romanelli, ${ }^{1}$ Alice Pressman ${ }^{1}$

\section{${ }^{1}$ Sutter Health, Walnut Creek, CA}

Background: Our existing virtual data warehouse (VDW) is confined to a single site in a large integrated health care network in Northern California. We sought to expand our VDW to multiple sites to incorporate data on a more diverse population. This allows our researchers to study a variety of data and collaborate with health care systems across the nation and within HCSRN.

Methods: Our VDW is based on an existing data warehouse that was built using a Star Schema model (specifically Kimball methodology). We built the schema for 1 site that has outpatient clinics covering approximately 1 million lives each year. We utilized the existing curated data warehouse to transform the data to meet VDW specifications. Records from multiple sites were de-duplicated. Billing records and encounters were used to impute utilization and enrollment. The single-site VDW resided on a virtual server. Because the extract, transform, load (ETL) processes for data from 5 sites consumed much larger quantities of memory than the processes required for a single site, the virtual server was no longer adequate. Therefore, we required a dedicated physical server to host our large-scale VDW development.

Results: After adding 4 other sites, for years 2000 through 2018, total enrollment count based on utilization increased by $120 \%$. Total HMO count increased by $72 \%$. Proportions of patients self-identifying as African American, other race, and white race increased by 2, 5, and 12 percentage points, respectively, while those proportions for patients identifying as Asian decreased by 7 percentage points. Hispanic and nonHispanic population increased by 5 and 8 percentage points, respectively. There was no difference in the proportion of patients' age group and gender with the addition of new sites. Conclusion: The expanded VDW now provides a much larger, more diverse data set for researchers. We have enhanced capabilities to do studies on diverse populations and collaborate with other sites across the nation. Next steps are to incorporate hospital inpatient data.
P5.04

Tools for Virtual Data Warehouse Master Data Management

John Weeks, ${ }^{1}$ Dustin Key, ${ }^{1}$ David Cronkite ${ }^{1}$

${ }^{1}$ Kaiser Permanente Washington, Seattle, WA

Background: At Kaiser Permanente Washington's Health Research Institute, we have built a set of tools that work with HCSRN's virtual data warehouse (VDW) to provide ontologies and mechanisms for our counters and data marts based on models like i2b2 and OMOP to be exported from our VDW master data management. We would like to share our work in building a proactive master data management set of tools for our organization.

Methods: The VDW codes and relationship hierarchy ontologies are built using SAS. SAS is also the tool that generates the $\mathrm{i} 2 \mathrm{~b} 2$ and OMOP common data models. The counter interface is built using Python. We use these tools to make our data more flexible and accessible to the larger research community.

Results: After building the VDW master data management model, we used the infrastructure to empower us to generate several useful tools that have broad applications and support. Besides being able to present our VDW data in a clinical context, we are also able to generate counts that are useful in preparation for research. We also use this infrastructure to generate external data models such as i2b2 and OMOP common data models.

Conclusion: We have been able to illustrate the value of the improvement of our VDW master data management model and are excited to share the work that we have done with the HCSRN.

\section{P5.05}

Sex, Gender, and Sexual Minorities: New Virtual Data Warehouse Demographic Data

\section{Roy Pardee, ${ }^{1}$ Catherine Cleveland, ${ }^{2}$ Don Bachman, ${ }^{2}$} Mark Schmidt ${ }^{2}$

${ }^{1}$ Kaiser Permanente Washington, Seattle, WA; ${ }^{2}$ Kaiser Permanente Northwest, Portland, OR

Background: Since its inception, virtual data warehouse (VDW) conflated the concepts of sex and gender in a single variable, which is explicitly defined as an opaque combination of those concepts. As a result, end-users have not known what concept the table captured for any given patient. As the science of medicine and society begin to recognize the importance of these distinctions to patient care, new clinical workflows are making more nuanced data available at HCSRN organizations. This provides opportunity to at once cure a defect and enable new types of research with the VDW. 
Methods: After much discussion, research, and reconnaissance both of existing standards and data at member organizations, the VDW's Enrollment/ Demographics workgroup put together a package of data changes that disentangle these disparate concepts.

Results: The change proposal affects only the demographics table and consists of 2 parts: 1) deprecation and removal of the existing "gender" variable; and 2) adding 3 new variables to hold a) sex assigned at birth, b) administrative or legal sex, and c) gender identity. Sex assigned at birth is the only concept that is static - the other two can change over time. Because the VDW demographics table is not suited to tracking those changes, all 3 new variables hold the information as of the latest ascertainment (ie, the last time the table was rebuilt or updated).

Conclusion: In designing research data structures, there is a tension between adhering to established standards like the ones provided by Logical Observation Identifiers Names and Codes (LOINC) and the Centers for Disease Control and Prevention (CDC) Public Health Information Network Vocabulary Access and Distribution System (PHIN VADS) versus closely accommodating the data in hand at member sites. For this effort we favored the latter. As a result, only the gender identity variable is fully compatible with standards propounded by LOINC and the CDC. The new design makes a place for the data HCSRN organizations are actually collecting and should serve as a solid foundation going forward for both general purpose and sexual minority research projects.

\section{P5.06 \\ Development of a Venous Thromboembolism (VTE) Data Phenotype to Retrospectively Identify Cancer Patients With VTE}

Benjamin Andrick, ${ }^{1}$ Jesse Manikowski, ${ }^{1}$ Anna
Baughman ${ }^{1}$

${ }^{1}$ Geisinger Health, Danville, PA

Background: Venous thromboembolism (VTE) remains a major cause of morbidity and mortality in patients with cancer warranting further research. Yet retrospective identification of VTE events in electronic medical records (EMR) remains problematic due to inaccuracies of administrative coding alone. Previous studies demonstrated the addition of an ambulatory anticoagulant prescription criterion increases the likelihood of identifying true VTE events. We piloted the application of such a VTE data phenotype (VTE-related ICD coding and text + outpatient anticoagulation prescription) for patients with cancer.

Methods: Patients with cancer were primarily identified using the Oncology Cancer Registry, which is a statemandated electronic database of cancer cases extracted from the institutional EMR by certified tumor registrars. Inclusion criteria further required at least one computed tomography (CT) scan within 5 years of cancer diagnosis. This criterion was intended to mitigate the influence of incidental VTEs on true events. Subsequently, the VTE data phenotype was applied utilizing a combination of text and ICD codes to identify VTE-related diagnoses. Documentation of the VTE diagnoses was required on more than 2 distinct instances. Lastly, the VTE data phenotype required documentation of an ambulatory anticoagulation prescription within 30 days of the first documented VTE diagnosis. This provided two cohorts: patients with cancer with or without a VTE event. In order to be $80 \%$ confident that the percentage of patients within the cancer VTE group who truly experienced a VTE event was $>95 \%, 30$ random charts were extracted from each cohort for confirmation by manual chart adjudication.

Results: 54,612 patients were identified with cancer and a CT scan within 5 years of diagnosis between 2008 and 2018. Application of the above VTE data phenotype yielded 3176 cancer patients with and 50,388 without a VTE event. Manual chart review demonstrated a positive predictive value of $93 \%$ (28 of 30) for the cancer with VTE cohort and a negative predictive value (NPV) of 100\% (30 of 30) for the cancer without VTE cohort.

Conclusion: The combination of VTE-related administrative coding and text searches for VTE-related diagnoses with an ambulatory anticoagulation prescription can be utilized to increase the accuracy of retrospective identification of cancer patients with and without a VTE event.

\section{P5.07}

\section{Clinical Needs and Resource Use Among Patients} With Cardiometabolic Conditions

Xiaowei (Sherry) Yan, ${ }^{1}$ Sukyung Chung, ${ }^{1}$ Zijun Shen, ${ }^{1}$ Hannah Husby, ${ }^{1}$ Jake Delatorre-Reimer, ${ }^{1}$ Farah Refai, ${ }^{1}$ J.B. Jones ${ }^{1}$

\section{${ }^{1}$ Sutter Health, Walnut Creek, CA}

Background: Health care organizations often rely on cost of care as a measure to identify target populations for chronic disease management outreach. We previously developed a segmentation model using measures of clinical need to group patients with cardiometabolic conditions (ie, hypertension, hyperlipidemia, diabetes) into clinically meaningful, distinct segments. Segment 1 represents $10 \%$ of the cardiometabolic patient population with the highest prevalence of cardiovascular disease (CVD) risk and Segment 4 (50\% of cardiometabolic patients) the least. Segment 3 (25\% of cardiometabolic patients) had relatively low CVD risk but the highest prevalence of mental health conditions (ie, anxiety or depression) ( $34 \%$ vs $<20 \%$ in other segments). In this study, we examined whether high clinical needs, represented by segments, are associated with higher costs of care among patients with cardiometabolic conditions.

Methods: The study sample included 30,816 individuals who had 1) any cardiometabolic condition, 2) HMO insurance in 2016, and 3) were patients from Sutter Health, a large mixed- 
payer health care system in Northern California. Outpatient costs were measured with total relative value units (tRVUs), a standard measure used by Medicare and other payers in calculating reimbursement for physician services. Inpatient utilization was measured by the likelihood of hospitalization for any reason during the year. We compared average tRVUs and hospitalization rate per patient across the 4 segments.

Results: Segment 3 had the highest tRVUs (mean: 36.6 [standard deviation: 46.1]) followed by Segment 1 (32.1 [34.4]). Segment 2, despite its distinctly lower CVD risk compared to Segment 1, had only slightly lower tRVU (30.7 [47.5]) than Segment 1. Similar patterns were observed in hospitalization rates - highest in Segment $3(18.1 \%)$, followed by Segment 1 (16.8\%) and Segment 2 (13.8\%). Costs of care for Segment 4 were lowest, less than half of Segment 2 in both tRVUs (15.2 [20.8]) and hospitalization $(5.9 \%)$. All the differences in tRVUs and hospitalization rates across segments were statistically significant $(\mathrm{P}<0.01)$.

Conclusion: In this population of patients, clinical needs, as determined using our segmentation model, are strongly associated with health care costs. Mental health needs, in addition to CVD risk factors, should be recognized and addressed as they are a major contributor to our measured costs.

\section{P5.08}

\section{In the Beginning Was T, N, M...}

\section{Rick Krajenta, ${ }^{1}$ Lois E. Lamerato ${ }^{1}$ \\ ${ }^{1}$ Henry Ford Health System, Detroit, MI}

Background: Cancer staging provides a detailed descriptive classification of tumors in a simple manner, showing tumor size, lymph node involvement, and spread to distant sites. Surveillance, Epidemiology, and End Results (SEER) extent of disease is derived from clinical, radiologic, and pathologic components producing a scaled numeric value that convey information regarding invasiveness using the T, N, M local/ regional/distant classification (TNM). However, staging of cancer is not a fixed science, and while SEER TNM-based staging has remained constant since 1973, American Joint Commission on Cancer (AJCC) stages have changed and expanded over time. The objective is to illustrate how AJCC stages have evolved and to formulate a plan for incorporating the latest AJCC staging criteria (version 8) into the virtual data warehouse (VDW).

Methods: Two cancer sites will be used to illustrate required elements for AJCC staging across versions. Specific inputs of each version will show how the evolution of clinical care necessitates stage revision. The current VDW tumor table data structure will be assessed to determine what changes will be needed to accommodate the current AJCC structure.

Results: The first edition of the AJCC staging manual was published in 1977, for diagnoses 1978-1983. Subsequent revisions took place every 5 to 7 years with the most recent edition, AJCC 8, for cancers diagnosed 2018 or later. Incorporating biological factors began with AJCC 6 in 2004 and has expanded with successive editions as treatment and surveillance move toward a personalized perspective. Expanding prognostic elements beyond strictly anatomic descriptions results in site-specific factors and provides data for evaluation of new and future therapeutic targets. AJCC 8 includes additional tumor characteristics required for precision medicine standards. These factors add complexity to AJCC staging.

Conclusion: Incorporating prognostic factors with anatomic measurement for staging is mandatory. Tumors with identical anatomic characteristics and different hormone receptors would receive different treatment regimens and most likely exhibit different outcomes. The standards for staging that encompass all the information to characterize a tumor with respect to therapy and survival will continue to expand to a targeted treatment approach, while retaining original TNM, and need to be applied to VDW.

\section{P5.09 \\ Which Method Wins in a Battle of Clinical Prediction Tools? Machine Learning Versus Traditional Statistics}

\author{
Craig Wood, ${ }^{1}$ Jeremy Orr, ${ }^{2}$ Tal Sagiv, ${ }^{2}$ Adam Cook, ${ }^{1}$ \\ Lisa Bailey-Davis ${ }^{1}$
}

${ }^{1}$ Geisinger Health, Danville, PA; ${ }^{2}$ Medial EarlySign, Aurora, Colorado

Background: Health care systems are increasingly developing and adopting analytical strategies to identify populations with biological and social determinants of chronic disease. As the field of data science matures, the variety of analytical tools expands and quality of methods used for developing clinical prediction tools improves. Traditional statistical methods may result in simple tools that are easy to develop, whereas machine learning methods may be more complex. Each method requires variable investment of workforce and technology resources in the development and implementation stages to produce health outcomes and cost savings. This study compares tools derived from both methods and reviews situations in which you might choose one method over the other.

Methods: Clinical prediction tools were independently applied on the same study cohort using traditional statistical methods and machine learning. Study outcomes included measures of diagnostic test accuracy (sensitivity, specificity, accuracy, area under the curve) and measures of agreement for person-level predicted probabilities.

Results: The results are a work in progress. Preliminary results suggest improved accuracy for a tool derived from machine learning. Predicted probabilities will be assessed to determine the frequency of disagreements that were greater than clinically accepted limits. 
Conclusion: Machine learning and traditional statistical methods are both useful for creating clinical prediction tools. The choice of method likely depends on how the tool is implemented in clinical practice. Simple tools may be integrated directly into care to engage patients and providers in personalizing treatment decisions and offer whole population benefit over time. Machine learning tools that more accurately identify high-risk populations may be critical in comprehensive health care system initiatives that aim to efficiently and effectively improve health outcomes in the short term. Understanding the strengths and limitations of each method will guide future research methodology and ultimately improve care, health outcomes, and offer cost savings.

\section{MATERNAL, CHILD \& FAMILY HEALTH}

\section{OA10.01 \\ Teaching Appropriate Gestational Weight Gain}

A. Dhanya Mackeen, ${ }^{1}$ Danielle Downs, ${ }^{2}$ Vonda Hetherington, ${ }^{1}$ Vani Movva, ${ }^{1}$ Jennifer Savage,${ }^{2}$ Amanda Young, ${ }^{1}$ Lisa Bailey-Davis ${ }^{1}$

\section{${ }^{1}$ Geisinger Health, Danville, PA; ${ }^{2}$ Penn State University, State College, $P A$}

Background: Excessive gestational weight gain (GWG) is associated with adverse pregnancy outcomes (eg, gestational diabetes, cesarean), yet few women report awareness of appropriate GWG. We tested a health intervention designed to improve awareness and achievement of appropriate GWG. Methods: A randomized controlled trial was conducted at Geisinger Health in Pennsylvania where excessive GWG is common among women with preconception obesity. Eligible consenting participants with prepregnancy body mass index of $>30.0 \mathrm{~kg} / \mathrm{m}^{2}$ were randomized (1:1) to: 1) usual care (UC), consisting of written educational materials and counseling by their provider; or 2) enhanced care (EC) - UC plus a) personalized letter from a physician detailing appropriate GWG, b) exposure to individualized GWG chart via the patient portal, and c) registered dietitian nutritionist telehealth counseling (initial consult plus 10-20 minutes/1-2 weeks) until delivery. The primary outcome was the proportion of women that gained less than or equal to 20 pounds in pregnancy, per guidelines. We also assessed the number of patients that gained $11-20$ pounds and total GWG.

Results: A total of 224 women were analyzed: 110 in EC and 114 in UC. Groups were similar according to baseline characteristics: $<30$ years of age, white race, non-Hispanic ethnicity, college-educated, $<\$ 75,000$ annual income, and not nulliparous. Of EC participants, 69\% received study-related registered dietitian nutritionist counseling; additionally, 25\% of EC and $30 \%$ of UC participants received non-study-related registered dietitian nutritionist care (eg, gestational diabetes).
There were no significant differences between the groups regarding the percentage of patients that gained less than or equal to 20 pounds in pregnancy: $40 \%$ in the EC arm vs $45 \%$ in the $\mathrm{UC}$ arm $(\mathrm{P}=0.49)$. This persisted regardless of adjusting for covariates. There were no significant differences between the groups with regards to GWG of $11-20$ pounds $(22 \% \mathrm{EC}$ vs $26 \% \mathrm{UC}, \mathrm{P}=0.53)$. Total $\mathrm{GWG}$ did not differ between the groups (21 pounds EC vs 17 pounds $\mathrm{UC}, \mathrm{P}=0.19$ ).

Conclusion: Enhancing prenatal care for appropriate GWG did not result in a greater percentage of patients gaining within the guidelines as compared to usual care, potentially explained by non-study-related exposure to registered dietitian nutritionists. Future planned analyses will evaluate effects on proximal outcomes and whether the intervention worked for subsets of the sample, accounting for potential moderating factors (eg, stress, sleep, food insecurity).

OA10.02

\section{Use of Electronic Health Records to Assess Prevalence of Anemia and Iron Deficiency in Pregnancy: A Pilot Study With Kaiser Permanente Northwest}

Joanna Bulkley, ${ }^{1}$ Kimberly Vesco, ${ }^{1}$ Anne Williams, ${ }^{2}$ Nicole Ford, ${ }^{2}$ Lindsay Jenkins ${ }^{1}$ Andrea Sharma ${ }^{2}$

\section{${ }^{1}$ Kaiser Permanente Northwest, Portland, OR; ${ }^{2}$ Centers for Disease Control and Prevention, Atlanta, GA}

Background: Anemia and iron deficiency are known to increase during pregnancy, but surveillance data necessary to target and evaluate interventions are lacking. Electronic health record laboratory data may serve as a data source for surveillance. Among first trimester pregnancies, we sought to describe the availability of laboratory test results from anemia screening and iron status evaluation and to estimate population prevalence of anemia, iron deficiency, and irondeficiency anemia (IDA; ie, presence of both anemia and iron deficiency).

Methods: We identified pregnancies to women, $\geq 18$ years old, that began and ended between 2005 and 2016. To assess first trimester screening and subsequent diagnostic testing of iron status, we analyzed pregnancies for which 1) prenatal care started in the first 14 weeks gestation, and 2) the woman was continuously enrolled in Kaiser Permanente Northwest for the first 20 weeks or entire pregnancy if ended before 20 weeks gestation $(n=41,991)$. From clinical and research laboratory databases, we selected the earliest lab result for hemoglobin or hematocrit within 14 weeks gestation and the earliest lab result for ferritin within 20 weeks gestation. Anemia was defined as hemoglobin of $<11.0 \mathrm{~g} / \mathrm{dL}$ or hematocrit of $<33.0 \%$. Iron deficiency was defined as ferritin of $<15.0 \mathrm{ug} / \mathrm{L}$. Results: Among eligible pregnancies, 93\% $(n=38,923)$ had hemoglobin and hematocrit test results. The first prenatal visit and hemoglobin/hematocrit test result were both at 8.7 weeks on average. Anemia prevalence was 3\% $(n=1045)$. 
Few eligible pregnancies had laboratory test results for ferritin. Among pregnancies with anemia, 15\% $(n=156)$ had a ferritin result; of these, $49 \%$ had iron deficiency. Only 3\% of pregnancies without anemia had a ferritin result $(n=1112)$; of these, 24\% had iron deficiency. Based on 1268 pregnancies with both anemia and iron deficiency assessed, prevalence of iron deficiency and IDA was $23 \%$ and $6 \%$, respectively, but estimates may not be representative due to selective screening. Conclusion: Because anemia screening is nearly universal at prenatal care onset, electronic health record data has the potential to monitor anemia prevalence and trends. However, few women were evaluated for ferritin, limiting the ability to obtain representative estimates of iron deficiency or IDA. Additional efforts are needed to determine if evaluation of iron deficiency can be improved at the health system level and whether this improves health outcomes.

\section{OA10.03}

Prioritizing Distal Determinants of Maternal Health and Improving Their Measurement Through Validation Research in Argentina, Ghana, and India

\section{Rima Jolivet, ${ }^{1}$ Richard Adanu, ${ }^{2}$ Eduardo Bergel, ${ }^{3}$ Niranjan Saggurti, ${ }^{4}$ Delia Bandoh, ${ }^{2}$ Amanda Mabel Berrueta, ${ }^{3}$ Suchandrima Chakraborty, ${ }^{4}$ Julia Cohn, ${ }^{1}$ Sarah Huber- Krum, ${ }^{1}$ Magdalene Odikro, ${ }^{2}$ Veronica Pingray, ${ }^{3}$ Sowmya Ramesh, ${ }^{4}$ Ernest Kenu, ${ }^{2}$ Ana Langer, ${ }^{1}$ Jewel Gausman ${ }^{1}$ \\ ${ }^{1}$ Women and Health Initiative, Harvard T.H. Chan School of Public Health, Boston, MA; ${ }^{2}$ School of Public Health, University of Ghana, Accra, Ghana; ${ }^{3}$ Instituto de Efectivdad Clinica y Sanitaria, Buenos Aires, Argentina; ${ }^{4}$ Population Council India, New Delhi, India}

Background: In 2015, the World Health Organization (WHO) released "Strategies toward ending preventable maternal mortality" (EPMM Strategies), which elaborates 11 key themes, emphasizing a systemic approach and increased attention to the full spectrum of determinants of maternal health and survival. Following the release of the EPMM Strategies, a set of indicators to track progress in these areas was identified to measure key leverage points at the health system, health policy, and population levels for tracking progress toward achieving the strategic objectives outlined in the EPMM Strategies (Jolivet et al, 2018). This presentation describes research to validate 10 policy and health systemlevel indicators from the EPMM monitoring framework. The specific aim of this study is to improve maternal health indicators that are not routinely collected and reported at a national level in 3 countries using a mixed-method, observational study design. The research is being carried out by the Harvard T.H. Chan School in collaboration with partners in Argentina, Ghana, and India.

Methods: This observational study utilizes a mixed-methods research approach, including quantitative and qualitative primary prospective data collection, retrospective review of facility-level patient and administrative data, and crosssectional review of secondary policy, legal, and regulatory data. A specific methodology is articulated for validating each indicator, based on the underlying construct it measures and its specific metadata. We will present the methodology for each validation exercise from the research protocol in finalization. Results: Validation research on health system- and policylevel indicators is not common, therefore, this work contributes new knowledge on validation methodology to the field as well as helps to substantiate the definitional framework and guidance under development by WHO's MoNITOR expert working group. For each indicator, specific methods have been designed to explore validation questions directed at testing various types of validity: construct validity, content validity, and convergent validity.

Conclusion: Improving measurement is a crosscutting action required to improve health systems to end all preventable maternal deaths. However, developing robust indicators is a challenge; indicator validation research is important in this era of increased demand for quality, reliable data to measure distal determinants of maternal health.

\section{OA10.04}

\section{Development of an Equity Dashboard to Monitor Health Disparities in Women's Health}

\section{Jordan Hastie ${ }^{1}$}

\section{${ }^{1}$ Highland Hospital, Alameda Health System, Oakland,} CA

Background: The American College of Obstetrics and Gynecology has identified at least 15 areas of inequities in women's health and health care among minority women. Identifying disparities via measuring and monitoring health outcome data is fundamental to ensuring quality and informed care. Clinical dashboards are used to display outcome data in a visual, concise format that allows for the identification of gaps in care. Information gleaned from dashboards can be used as a tool to guide institutional action and aid in mobilizing health care providers to improve care. Safety-net hospitals serving vulnerable populations tend to have limited fiscal resources and would have the most to gain from such information. Discovering previously unknown gaps in care also has cost savings potential, as improving outcomes can prevent costly downstream consequences of delayed or inadequate health care.

Methods: We developed a dashboard that evaluates disparate health outcomes and access across 6 indicators of vulnerability: race, ethnicity, gender, age, language, and zip code. The areas measured include rates of preterm birth, fetal demise, cesarean sections, cervical cancer, minimally invasive hysterectomy and myomectomy, and receipt of longacting reversible contraception. Where possible, the data are reported alongside the county, state, and national average.

Results: Among African American women, the preterm birth 
rate ranged from $9.6 \%$ to $13.0 \%$; the rate was highest among African American women at all time points. The cesarean section rate ranged from $28.7 \%$ to $33.3 \%$; similarly, the rate was consistently highest among African American women. On average, both the rates of preterm birth and cesarean section were $34 \%$ higher than the rate of all other women from 2014 to 2019 (8.3\% and $22.9 \%$, respectively).

Conclusion: The equity dashboard provides a concise view of key health inequities that allows us to identify trends, gaps, and opportunities to improve patient outcomes and access to services with the overall goal of eliminating disparities within our institution. In the future, these data could be further stratified by other measures, such as income, migratory status, education, and housing security, to guide future quality improvement projects and find more opportunities to creating equal care for all.

\section{P11.01}

\section{A Retrospective Analysis of the Relationship Between Race/Ethnicity, Age at Delivery, and the Risk of Gestational Diabetes Mellitus}

\section{Bin Liu, ${ }^{1}$ Lois E. Lamerato, ${ }^{1}$ Dawn Misra ${ }^{2}$ \\ ${ }^{1}$ Henry Ford Health System, Detroit, MI; ${ }^{2}$ Wayne State University, Detroit, MI}

Background: Many factors have been reported to be associated with developing gestational diabetes mellitus (GDM); however, few have been studied in depth. This study aimed to investigate the relationship between maternal race/ ethnicity, age at delivery, and the risk of GDM.

Methods: Patients of Henry Ford Health System who delivered a live singleton child and were diagnosed with or without GDM during 2010-2015 were included. Maternal race/ethnicity, age, body mass index (BMI), parity, GDM in previous pregnancy, smoking status, and insurance membership were collected from the electronic health records. Neighborhood median family income data were obtained from U.S. Census Bureau. Logistic regression analysis was performed to explore the effects of maternal race/ethnicity and age at delivery on the GDM outcome after adjusting for covariates, including BMI, parity, previous GDM, smoking status, and neighborhood family income.

Results: There were 16,258 women included in the study. Of those, 1801 women (12.5\%) were diagnosed with GDM. Delivery at an older age ( $\geq 23$ years) was associated with a significantly higher risk of a GDM diagnosis than at a younger age ( $<23$ years) — odds ratio (OR): 2.24 (95\% CI: $1.84-2.73$ ) vs OR: 5.02 (95\% CI: 4.18-6.03) - however, the risk was not as profound in African American women (OR: 1.65) compared to non-African American women (OR: 2.07). In a multivariable model controlling for age, BMI, parity, previous GDM, smoking status, and neighborhood family income, the risks of a GDM diagnosis were significantly higher in Asians (OR: 2.81 [95\% CI: 2.28-3.48]), Hispanics (OR: 1.27
[95\% CI: 1.05-1.55]), and Arab Americans (OR: 1.46 [95\% CI: 1.20-1.78]) and lower in African Americans (OR: 0.64 [95\% CI: 0.56-0.74]), as compared to whites.

Conclusion: Compared to whites, Asians, Hispanics, and Arab Americans have higher risk and African Americans have lower risk of a GDM diagnosis. Delivery at an older maternal age increases the risk of GDM diagnosis. Race/ethnicity moderates the association between older maternal age and risk of GDM diagnosis. This study provides information for public health professionals, health practitioners, and pregnant women to be aware of and better understand the risk of GDM as related to race/ethnicity and maternal age.

\section{P11.02 \\ Pain Management Related to Wisdom Teeth Extractions: Interviews With Teens and Parents}

\author{
Shannon G. Mitchell, ${ }^{1}$ Anjali R. Truitt, ${ }^{2}$ Lauryn Davin, ${ }^{2}$ \\ D. Brad Rindal ${ }^{2}$
}

${ }^{1}$ Friends Research Institute, Inc., Baltimore, MD; ${ }^{2}$ HealthPartners, Bloomington, $M N$

Background: Despite growing concern about the opioid epidemic, the number of opioid prescriptions for 11-18-yearold dental patients increased from 2010 to 2015. Prescribed opioid use before completing high school graduation is independently associated with future opioid misuse among low-risk children. The majority of dental providers report prescribing opioid medications following wisdom teeth extractions. This study explored how adolescent patients and their parents/guardians make decisions about opioid use for pain management after dental extractions.

Methods: Participants were identified based on age and recent dental extraction procedure. A notification letter was sent to the parent/guardian of the patient, and a follow-up call was made a week later. Verbal consent by the parent/guardian and verbal assent by the patient was obtained. A trained interviewer conducted paired structured interviews with interview guides tailored to the respondent (eg, parent/guardian or patient). Interview guides addressed patient- and family-centered decision-making related to pain management. All interviews were conducted by phone, audio-recorded, and transcribed. De-identified data were analyzed using qualitative analysis software using a directed content analysis approach.

Results: All participants interviewed (15 patients, 15 parents) reported receiving an opioid prescription following wisdom teeth extractions. Of those who did take the opioid prescription, most only took it the day of the extraction and up to 2 days following. This appeared to be common regardless of whether the adolescent, parent, or a shared pain management decision-making approach was taken. Few patients took all of it, and several took none. In addition to conversations with their dentist, most patients and their parents discussed pain management plans before or following the extraction, which included nonmedication options, over-the-counter 
medications, and opioid prescriptions as needed. No one reported significant side effects from any of the options. Most patients and their parents felt that the over-the-counter medication was adequate in managing pain.

Conclusion: Opioid prescribing is common following wisdom teeth extractions. Providers may have an opportunity to reduce the number of days for which opioids are prescribed and reduce the number of opioid prescriptions. When opioids are prescribed, providers should educate patients and their parents/guardians about safe disposal to mitigate the potential for diversion.

\section{HEALTH CARE DELIVERY \& COVERAGE}

\section{OA12.01 \\ Adherence to Best Practices for Medication Reconciliation Identified Through Observations in Primary Care Practices}

Michael Gionfriddo, ${ }^{1}$ Allison Middernacht, ${ }^{1}$ Vanessa Duboski, ${ }^{1}$ Melissa Kern, ${ }^{1}$ Eric Wright ${ }^{1}$

\section{${ }^{1}$ Geisinger Health, Danville, PA}

Background: Medication lists contained within electronic health records (EHR) should reflect patients' current medications; however, these lists are rarely complete and accurate. Best practices for medication reconciliation exist, but fidelity to these procedures in primary care settings by staff rooming patients and providers are unclear. We sought to quantify the extent to which best practice procedures for medication reconciliation are followed in routine primary care practices.

Methods: We conducted an ethnographic study of nurses and medical assistants rooming patients in primary care clinics in a health care delivery network from June through September 2019. Practice sites and rooming staff were selected in collaboration with clinic leadership on a volunteer basis. All sites that volunteered were included. Convenience sampling was used to conduct individual observations. Staff at the sites were not blinded to observations. Study team members documented time spent on medication reconciliation and actions of rooming staff conducting medication reconciliation using a predeveloped checklist of best practices collected from a search of the literature.

Results: We observed 170 patient encounters with 43 nurses/ medical assistants across 15 primary care clinics. On average, the staff took approximately 2 minutes (standard deviation: 2 minutes; range: $<1$ minute-14 minutes) to conduct a medication reconciliation and completed approximately $50 \%$ (range: $30 \%-80 \%$ adherence) of the behaviors considered to be part of an optimal medication reconciliation. In all encounters observed, staff accessed the medication list, reviewed medications, and documented that they reviewed the medication list, with nearly all staff querying patients to verify current medications (99\%), verify if patients take medications as prescribed (99\%), and review the names of medications (99\%). Few staff used EHR functionality to view medications from other locations (ie, reconcile outside medications [7.6\%]) or queried the patient about non-Geisinger Health medications (26\%), over-the-counter $(36 \%)$ or as-needed medications $(24 \%)$, adherence $(5 \%)$, side effects $(12 \%)$, and portions of SIG instructions (eg, route [2\%], indication [13\%]).

Conclusion: Medication reconciliation is highly variable, and several of the components of conducting a thorough medication reconciliation are not routinely collected within a primary care visit with rooming staff. Future studies should assess reasons for the lack of completion.

\section{OA12.02}

"6-Clicks" Short Forms: Effects on Discharge Location, Length of Stay, and Readmission in Hospitalized Acute Care Patients Receiving Rehabilitation

Melissa Harry, ${ }^{1}$ Theo Woehrle, ${ }^{1}$ Colleen Renier, ${ }^{1}$ Margaret Furcht, ${ }^{1}$ Michelle Enockson ${ }^{1}$

\section{${ }^{1}$ Essentia Health, Duluth, MN}

Background: Accurately predicting appropriate discharge location for hospitalized patients may help reduce costs related to rehabilitation and hospital readmissions. The Activity Measure for Post-Acute Care (AM-PAC ${ }^{\text {TM}) ~ " 6-C l i c k s " ~}$ Inpatient Basic Mobility and Daily Activity Short Forms are standardized functional assessment tools that may assist physical and occupational therapists with better determining patient discharge location when implemented in clinical care during a hospital stay. The aim of this study was to evaluate the effect of 6-Clicks usage on discharge location, length of stay, and 30-day readmissions for adult patients receiving rehabilitation services during an acute inpatient hospital stay in 5 Essentia Health hospitals. Essentia is a predominantly rural integrated health care system with locations in Minnesota, North Dakota, and Wisconsin.

Methods: This retrospective observational study used electronic health record data. The sample included 12,314 hospital admissions (10,316 patients) from the 12 months prior to 6-Clicks implementation (June 1, 2015-May 31, 2016) and 13,241 hospital admissions (10,931 patients) from the year after full 6-Clicks implementation (January 1, 2017-December 31, 2017) within 5 Essentia hospitals. Analyses included descriptive statistics, areas under the receiver operating characteristic curves (AUC), logistic regression for initial hospitalizations, and negative binomial and binary logit-linked generalized estimating equations for correlated data.

Results: In 4 randomly drawn samples, the AUC for Basic Mobility and Daily Activity scale scores were 0.86 and 0.84 0.85 , respectively, for discharge to home or to an institution, 
including skilled nursing facilities. Scores also independently correctly classified more discharges to home than to other institutions. A 1-point increase in Basic Mobility and Total Activity scale scores was significantly associated with higher likelihoods of patients being discharged home, regardless of model tested. However, few significant differences were seen regarding length of stay or 30-day readmissions between preand post-6-Clicks cohorts, including by individual hospital.

Conclusion: The AM-PAC 6-Clicks Basic Mobility and Daily Activity Short Forms performed similarly to research published by the developers when distinguishing between discharge locations, supporting use by physical and occupational therapists in discharge planning. Future research could assess where additional intervention or staff training on the use of 6-Clicks may be needed to reduce length of stay and 30-day readmissions for hospitalized acute care patients receiving rehabilitation services.

\section{OA12.03}

\section{Association Between All-Cause Hospital Readmissions and Routine Vaccination Among Persons With Chronic Obstructive Pulmonary Disease in the LEAP Study}

\author{
Djeneba Audrey Djibo, ${ }^{1}$ Jessica Stempel, ${ }^{1}$ Andres Mora \\ Carpio, ${ }^{1}$ Darina Chudnovskaya, ${ }^{1}$ Jean G. Ford ${ }^{1}$
}

\section{${ }^{1}$ Einstein Healthcare Network, Philadelphia, PA}

Background: Poorly managed chronic obstructive pulmonary disease (COPD) can lead to repeated exacerbations and frequent acute hospital stays. Recommended vaccinations, such as the yearly influenza vaccine and the pneumococcal vaccine, while preventing or lowering the severity of the disease, also reduce the number of acute episodes. Identifying missed opportunities for upstream prevention of excess acute health care utilization in a vulnerable population is key to improving outcomes.

Methods: The LEAP study developed a retrospective cohort of asthma/COPD patients in a large urban safety-net hospital to examine environmental and social factors affecting health care utilization and to identify opportunities for intervention. Electronic health records were used to identify COPD cases using disease-specific ICD-9 and ICD-10 codes. Demographic information including male/female sex, ethnic group, and age also were collected. Information on hospital admissions was collected for 2008-2018, evidence of influenza and pneumococcal vaccination was recorded, and 30-day all-cause hospital readmissions were identified. Logistic regression was used to examine the association between readmission and vaccination status.

Results: COPD cases $(\mathrm{N}=1,068)$ were $60 \%$ female, were $80.3 \%$ African American, and had median age of 67 years. Evidence of influenza and pneumococcal vaccination was found in $64.2 \%$ and $60.5 \%$ of cases, respectively. Female COPD cases were more likely to have received influenza vaccination compared to male COPD cases, $67.8 \%$ vs $59.2 \%$ $(\mathrm{P}=0.006)$; however, there were no gender differences in the receipt of pneumococcal vaccination. Pneumococcal vaccination was not independently associated with 30day readmission $(\mathrm{P}=0.1)$. However, evidence of influenza vaccination was independently positively associated with decreased odds of 30-day hospital readmission $(\mathrm{P}=0.04)$. After controlling for age, gender, and ethnicity, the odds of readmission remained lower among those who had received influenza vaccination $(\mathrm{P}=0.007)$.

Conclusion: There is clear evidence that primary preventive measures, such as recommended vaccinations, may help reduce acute health care utilization among individuals with COPD. However, our study showed that the protective effects of pneumococcal vaccination do not extend to the risk of readmission. Further investigation of the differences observed, including the effects of social environment, will help understand the determinants of readmission to this urban safety-net hospital.

\section{OA12.04}

Improving Care Transitions Between Hospital and Home: How Do High-Performing Hospitals Do It?

Kimberly Fisher, ${ }^{1}$ Susanne Muehlschlegel, ${ }^{2}$ Timothy Konola, ${ }^{1}$ Sarah Bloomstone, ${ }^{1}$ Sybil Crawford,${ }^{1}$ Kathleen M. Mazor ${ }^{1}$

${ }^{1}$ Reliant Medical Group/University of Massachusetts Medical School/Fallon Health, Worcester, MA; ${ }^{2}$ University of Massachusetts Medical School, Worcester, MA

Background: The transition from hospital to home is an extremely high-risk time, with many patients experiencing adverse events. We sought to identify approaches used by hospitals that consistently achieve excellent care transitions (high-performers) but not by low-performing hospitals.

Methods: We conducted in-depth interviews with key stakeholders from nonspecialty acute care hospitals. Hospitals were classified as high- or low-performers based on the care transition measure (CTM-3) from the Hospital Consumer Assessment of Healthcare Providers and Systems (HCAHPS) Survey. Interviews were audio-recorded and transcribed verbatim. Themes were identified through iterative readings of the transcripts, generation of preliminary lists of themes, and discussion until the team reached consensus that all important themes had been identified. All transcripts were coded by 3 researchers. Differences in coding were discussed until consensus was achieved.

Results: A total of 39 stakeholders from 9 high-performing hospitals and 9 stakeholders from 3 low-performing hospitals participated. Hospitals were diverse with regard to size, teaching status, and geographic location. Participants had varied roles (frontline [n=11], manager/director [ $\mathrm{n}=27]$, senior leadership [n=10]) with backgrounds in nursing, social work, case management, administration, pharmacy, 
and medicine. Qualitative analyses revealed 3 domains that characterized care transition practices: processes, structural elements, and contextual factors. Each domain was comprised of several themes. Care transition processes that distinguished most high- from low-performers included assessment of discharge needs among all or nearly all patients; targeting intensive care transition initiatives to patients at higher risk of readmission or death; emphasis on patient education; and pharmacy involvement in medication reconciliation, medication education, and ensuring patients receive discharge medications. Structural elements included staffing, availability of resources in the community, and hospital features such as size. Preliminary analysis suggests that contextual factors are especially important in distinguishing high- from low-performing hospitals. High-performing hospitals were characterized by an extremely patient-centered culture, a nonhierarchical and collaborative workplace, and a commitment to continual improvement in which frontline staff are consistently engaged. Hospital leaders were viewed as playing an important role in fostering these contextual factors.

Conclusion: These findings illustrate care transition practices that characterize high-performing hospitals that could be used by hospitals seeking to improve their own care transition performance.

\section{P8.01}

Knowledge and Awareness of a Value-Based Insurance Design Benefit Program for Diabetes Patients

Vanessa Simiola, ${ }^{1}$ Tiana N. M. Koch, ${ }^{1}$ Andrea Altschuler, ${ }^{2}$ Timothy B. Frankland, ${ }^{1}$ Alyce S. Adams, ${ }^{2}$ Deborah Taira, ${ }^{3}$ Dennis Ross-Degnan, ${ }^{4}$ Connie Trinacty ${ }^{1}$

${ }^{1}$ Kaiser Permanente Hawaii, Honolulu, HI; ${ }^{2}$ Kaiser Permanente Northern California, Oakland, CA; ${ }^{3}$ University of Hawai'i at Hilo, Hilo, HI; ${ }^{4}$ Harvard Pilgrim Health Care, Boston, MA

Background: Increasingly popular value-based insurance designs (VBIDs) have been shown to improve short-term adherence to medications for chronic illness with no increase in total spending. Kaiser Permanente Hawaii (KPHI) offers WellRx - a drug rider benefit that automatically waives all copays for essential medications and supplies for diabetes to select large employer accounts. Gaps in knowledge and awareness of these programs may lead to variation in program efficacy. This multimethod study evaluates the knowledge and awareness of a real-world VBID program at the employer and employee levels.

Methods: Semi-structured interviews were conducted with 11 employer representatives from $6 \mathrm{KPHI}$ accounts that participate in WellRx. Eligible employer participants were primary decision makers for health care benefits or advocates for health and wellness for their employees. Responses were reviewed independently by 3 of the authors. Thematic analysis was used to evaluate the employers' awareness of WellRx, methods for disseminating information about the program to employees, and the value of health and wellness. Mailed surveys were collected from 316 WellRx enrollees with diabetes currently receiving benefits from an interviewed employer account. Patients were asked about their knowledge of receiving a zero-dollar copay for diabetes medication.

Results: One-third of employer representatives were unaware of the WellRx benefit in their service agreement with KPHI. Prioritization of health was less frequent among these employer groups, particularly when compared to their perception of the importance of costs for health benefits. These employers less frequently reported engaging in discussions with their employees about health benefits and overall wellness. Of employer representatives who were aware of offering WellRx, several noted obstacles in distributing information about the program to their employees directly (ie, lack of employee engagement, low health literacy, communication barriers). About $30 \%$ of surveyed WellRx beneficiaries were unaware of filling their diabetes medications without paying copays.

Conclusion: Knowledge and awareness of available health care benefits vary at both employer and employee levels. Barriers to dissemination of information about health benefits by employers and gaps in patient-reported knowledge of a zero-copay program may be limiting, but common, factors in evaluating real-world VBID programs. System-based strategies may address variations in knowledge dissemination across consumer, payer, and organizational contexts.

\section{EPIDEMIOLOGY AND POPULATION HEALTH IMPROVEMENT}

P6.01

Prescription Opioid Use and Incident Psychiatric Disorders: An HCSRN Partnered Research Protocol

Lisa Matero, ${ }^{1}$ Brian Ahmedani, ${ }^{1}$ Lynn DeBar, ${ }^{2}$ Sarah Gebauer, ${ }^{3}$ Joanne Salas, ${ }^{3}$ Patrick Lustman, ${ }^{4}$ Mark Sullivan ${ }^{5}$

${ }^{1}$ Henry Ford Health System, Detroit, Ml; ${ }^{2}$ Kaiser Permanente Washington, Seattle, WA; ${ }^{3}$ Saint Louis University, St. Louis, MO; ${ }^{4}$ Washington University School of Medicine, St. Louis, MO; ${ }^{5}$ University of Washington, Seattle, WA

Background: Opioid analgesic use (OAU) is associated with an increased risk for depression even after controlling for the contributions from pain. Herein we describe a research collaboration between Saint Louis University and Henry Ford Health System that aims to determine the risk factors, either preceding or following OAU, that increase risk for incident depression and to systematically characterize the depression 
profile (ie, major depressive episode, dysthymia, bipolar, anhedonia, and vital exhaustion) associated with OAU and opioid use disorder.

Methods: This is a prospective cohort study of 1500 (750 Saint Louis, 750 Henry Ford) adult (age: 18-70 years) prescription opioid users invited to participate when they completed a new period (no OAU in previous 3 months) of 30-day OAU and have prescriptions allowing for 60 days of continuous opioid use. Targeted subject recruitment using electronic health record data is used to identify eligible subjects. Baseline, 6- and 12-month web or telephone survey will obtain data on lifetime and current psychiatric diagnoses, trauma, sleep disorder, social support, noncancer pain, and OAU. Subjects will be asked to complete 12 monthly assessments measuring pain-related functioning, depression symptoms, and OAU.

Results: Efforts to reduce morbidity and mortality associated with the opioid epidemic have led to significant declines in the number and duration of new opioid prescriptions written in the United States. These changes have challenged our ability to identify eligible subjects and required adapting the eligibility criteria to reflect current prescribing patterns. We now allow tramadol to count as an eligible opioid and do not require a chronic pain diagnosis. We have completed programming of a diagnostic interview in RedCap, making it the first available web-based, lay-administered, psychiatric diagnostic survey. Recruitment is scheduled to begin November 2019.

Conclusion: Restrictions on type of opioid and duration of new opioid prescriptions have dramatically increased the difficulty involved in obtaining eligible patients. Fewer eligible subjects per week has led to allowing for a 24-month baseline enrollment period. Allowing tramadol to count toward OAU greatly increased the number of subjects eligible for study and ensures that results will be relevant to current prescribing practices. Preliminary recruitment information and lessons learned will be discussed.

\section{P6.02}

Long-Term Trends and Risk Factors of Deaths From Traumatic Injuries at a Rural Wisconsin Level 2 Trauma Center

\section{David McClure ${ }^{1}$}

\section{${ }^{1}$ Marshfield Clinic, Marshfield, WI}

Background: Most studies of deaths from traumatic injury are from urban level 1 trauma centers. In contrast, rural areas have higher incidence of traumatic fatal injuries than urban areas. Nationally, there is also a recent overall trend of increasing numbers and rates from deaths due to traumatic injury. Therefore, the objective was to describe trends of trauma mortality and risk factors from a largely rural population.

Methods: This was a retrospective cohort study of injured patients admitted to a rural Wisconsin level 2 trauma center from 2000 through 2018. Details on medically attended injuries and deaths were obtained from the trauma registry. Fatality counts and proportions were described by year, gender, age, mechanism of injury, intent of injury, injury severity score (ISS), and length of hospital stay (in days). Survival analysis was used to explore major risk factors related to injury mortality.

Results: There were 17,556 events among 16,696 patients admitted from 2000 through 2018. The proportion of injuries caused by falls increased from $35 \%$ in $2000-2005$ to $52 \%$ in 2014-2018. The proportion caused by motor vehicle crashes decreased over time, from $31 \%$ to $18 \%$, respectively. The proportions due to other injury mechanisms were relatively stable, with very few due to firearms $(<1 \%)$. Overall fatality rates at the trauma center were 6\% from 2000 to 2004 and decreased to $4 \%-5 \%$ thereafter. The highest fatality rates were patients with ISS of $>15$ (fatality: 14\%) and those caused by firearms (fatality: 25\%). In the survival analysis, major risk factors for death were age of $>64$ years (hazard ratio [HR]: 2.4, 95\% CI: $1.3-4.3$ ); ISS of $>15$ (HR: $6.9,95 \%$ CI: $5.4-8.9$ ), falls (HR: 2.5, 95\% CI: 1.6-3.7), and firearms (HR: 10, 95\% CI: 6.2-17).

Conclusion: Long-term trends of traumatic injury mortality were similar to national trends, particularly in increasing proportion due to falls. However, the proportion of firearms deaths at this rural trauma center was low compared to urban or other rural centers. There were limitations of no available information on immediate deaths at the scene and loss to follow-up after discharge date. These will be addressed in a population-based follow-up study.

\section{P15.01}

Effects of a Pharmacist-led Program to Reduce Opioid Use: Results from a Natural Experiment

John Dickerson, ${ }^{1}$ Jennifer Kuntz, ${ }^{1}$ Xiuhai Yang, ${ }^{1}$ Mathew Slaugher, ${ }^{1}$ Jennifer L. Schneider, ${ }^{1}$ Alison Firemark, ${ }^{1}$ Dea Papajorgji-Taylor, ${ }^{1}$ Katherine Reese, ${ }^{1}$ Lou Ann Thorsness, ${ }^{1}$ Mark Sullivan, ${ }^{2}$ Lynn DeBar, ${ }^{3}$ David H. Smith ${ }^{1}$

${ }^{1}$ Kaiser Permanente Northwest, Portland, OR; ${ }^{2}$ Psychiatric and Behavioral Sciences, University of Washington School of Medicine, Seattle, WA; ${ }^{3}$ Kaiser Permanente Washington, Seattle, WA

Background: Levels of opioid prescribing and the attendant risks have become an urgent national concern. To address this, Kaiser Permanente Northwest (KPNW) implemented a pharmacist-led program ("STORM") in 2010 that held individual training for primary care providers (PCP) on contemporary opioid prescribing and STORM services. Once trained, PCPs could access STORM services to assist patients with opioid-exposure reduction. The PCP training was rolled out nonsystematically, allowing an opportunity to estimate causal effects of the program on PCP panel-level outcomes across time. 
Methods: Using KPNW electronic health record data and training records, we identified 331 PCPs who had active panels ( $\geq 100$ patients) and were employed at some time during the STORM training roll-out (2010-2012). We identified 379,378 adult patients paneled to these PCPs between 2007 and 2015 and assigned their monthly data to their paneled PCP. We then constructed a monthly PCP-level dataset with summary outcome measures (eg, average daily morphine equivalent $[\mathrm{MED}]$ ), potential confounders (eg, proportion of patients with chronic pain), STORM training details (eg, trained or not, months since training), and calendar time (to estimate secular trends). In order to empirically evaluate the nonsystematic dissemination, we estimated standardized differences between trained and untrained PCPs over time for likely confounders, specifically, the proportion of patients with chronic pain and average MED. We used generalized estimating equations to fit population-averaged models.

Results: We found standardized differences to be consistently below 0.2 between 2010 and 2012, offering reassurance of a nonsystematic dissemination. For average MED, we found an immediate effect following STORM training ( $\beta$ : $-0.301, \mathrm{P}<0.001$ ), average monthly reduction in the first year following training $(\beta$ : $-0.051, \mathrm{P}<0.001)$, and an average monthly reduction after the first year following training $(\beta:-0.052, \mathrm{P}<0.001)$. The estimated panel average MED 2 years after STORM training was $31.2 \%$ lower for trained PCPs compared to untrained PCPs. Other opioid summary outcomes showed similar results.

Conclusion: Our findings demonstrate that the STORM program is effective at reducing the average panel's opioid use. Next steps include examining subgroup effects and performing an economic evaluation.

\section{P15.02}

\section{Oral Health: An Important but Often Absent Component of Total Health Care}

Nancy P. Gordon ${ }^{1}$

\section{${ }^{1}$ Kaiser Permanente Northern California, Oakland, CA}

Background: Oral health is in an important component of total health. Poor oral health can lead to gum disease and chronic inflammation, increasing oral cancer risk and exacerbating management of cardiovascular conditions. Poor oral health can lead to tooth loss, making eating and speaking difficult and affecting emotional health. Despite the importance of oral health, health plans generally don't consider preventive dental care (PDC) as part of total health care. Patients are not routinely asked whether they are getting their teeth cleaned and examined or being advised to do so. Doctors seldom look into patients' mouths, let alone inspect teeth and gums for problems. Adults are recommended to get their teeth cleaned and examined at least annually, more often if at higher risk for oral health problems This study estimated the proportion of health-insured adults that did not receive PDC in the past
12 months and identified factors associated with lack of PDC. Methods: Pooled data from the 2011 and 2014/2015 cycles of the Kaiser Permanente Northern California (KPNC) adult Member Health Survey, weighted to the age-sex composition of 2014 KPNC membership, were used to estimate the percentages of adults, 25-34, 35-64, 65-74, and 75-85 years of age, with no PDC in the past 12 months. Lack of recent PDC was compared by sex, race/ethnicity, income, education, and health characteristics.

Results: Approximately $26 \%$ of adults in the $25-34$ and $75-$ 85 age groups and $18 \%$ of adults in the 35-64 and 65-74 age groups reported no PDC in the past year. Demographic and health factors were similar across age groups. Lack of PDC was more prevalent among blacks and Latinos than whites and increased as income, education, and self-rated health decreased. No recent PDC was reported by more than onethird of adults $65-85$ years of age with oral health problems that interfered with eating or talking, by one-third among $35-85$-year-olds, by approximately $30 \%$ of adults with diabetes, by $24 \%$ with heart disease and hypertension, and by $40 \%$ of smokers.

Conclusion: Many adults are not routinely getting preventive dental care, which may pose a health risk. Even when health plans are not offering this care, patients should be encouraged to get it and be given referrals to low cost services if needed.

\section{P15.03}

\section{Standardized Reporting of Chest Computed Tomography Results Improves Detection of Lung Cancer}

Jennifer Dusendang, ${ }^{1}$ Thomas Urbania, ${ }^{1}$ Stacey Alexeeff, ${ }^{2}$ Douglas Corley, ${ }^{1}$ Todd Osinski, ${ }^{1}$ Lori Sakoda, ${ }^{1}$ Lisa Herrinton ${ }^{1}$

\section{${ }^{1}$ Kaiser Permanente Northern California, Oakland, CA; ${ }^{2}$ Kaiser Permanente Mid-Atlantic States, Rockville, MD}

Background: The number of chest computed tomography (CT) scans performed each year is increasing, and standardization of the management of incidental lung nodules as recommended by the Fleischner Society remains important. Between 2015 and 2017, Kaiser Permanente Northern California rolled out a standardized reporting system that uses 7 tags (\#PUL0-\#PUL6) applied to chest CT reports to concisely convey nodule findings and recommended follow-up. Additionally, the tag "\#PUL5" indicates "suspicious for lung cancer" and automatically forwards the report to an expert multidisciplinary committee for case review.

Methods: To assess the effectiveness of the standardized reporting system, we performed a stepped-wedge analysis to retrospectively compare the incidence of lung cancer between patients with chest CT whose scan results were tagged versus those not tagged. We also computed performance characteristics of the \#PUL5 tag and performed chart review to assess potential areas for improvement in the reporting system. 
Results: We identified 99,148 patients with a chest CT between January 2015 and July 2017. Of these patients, $40 \%(n=39,409)$ had a tag applied to their radiology report, of which 2255 (5.7\%) were \#PUL5. Within 120 days of the initial CT scan, 2856 patients $(2.9 \%)$ were diagnosed with new primary lung cancer. Adjusting for patient demographics, Charlson comorbidity score, smoking status, previous cancer diagnosis, 6-month calendar period, and medical center, the odds of lung cancer diagnosis increased by $9 \%$ (95\% CI: $0-18 \%$ ) in the patients with tagged (vs untagged) reports. The odds of early stage lung cancer diagnosis increased by $24 \%$ (95\% CI: $9 \%-41 \%)$. Among patients with tagged reports, \#PUL5 had a sensitivity of $74 \%$, specificity of $96 \%$, and a number needed to diagnose of 2.7 for lung cancer. Opportunities for improvement related to too-literal distinctions between "nodule" and "mass" and limited radiologist knowledge of the patient's clinical context.

Conclusion: Standardized reporting and management of lung nodules was associated with an increased likelihood of diagnosing lung cancer, especially early stage lung cancer compared with prior unstructured reporting. Integrated health care systems have a unique opportunity to automate and standardize the management of suspicious findings on CT scans.

\section{$\mathrm{P} 15.04$}

Coordinator-Supported Hepatocellular Carcinoma Surveillance in Kaiser Permanente Mid-Atlantic States

Lauren Williamson, ${ }^{1}$ Venus Alert, ${ }^{1}$ Cabell Jonas, ${ }^{1}$ Eric Watson, ${ }^{1}$ Sundeep Basra, ${ }^{1}$ Michael Horberg ${ }^{1}$

\section{${ }^{1}$ Kaiser Permanente Mid-Atlantic States, Rockville, MD}

Background: Cirrhosis is a leading risk factor for hepatocellular carcinoma (HCC). Populations at risk for HCC that are candidates for surveillance generally include hepatitis B (HBV), hepatitis C, liver cirrhosis, nonalcoholic cirrhosis, and advanced liver fibrosis. The goal for HCC screening is to detect subclinical diseases at an earlier stage for at-risk patients through use of imaging results, which has a favorable effect on overall patient care outcomes and treatment options. Literature identifies barriers in adherence to HCC surveillance, which the Mid-Atlantic Permanente Medical Group (KPMAS) decided to act on when developing and building its HCC surveillance program. KPMAS's goal aims to determine how effectively a coordinator-supported approach with assisting physician improves adherence to not only HCC surveillance but to other chronic health programs. Methods: If eligible as a high-risk patient for developing HCC, a gastroenterologist (GI) will place a unique initial order to designate which lab work (eg, alpha-fetoprotein, hepatic function, or HBV DNA viral load) and imaging exam (ultrasound, magnetic resonance imaging, or computed tomography scan) to complete every 6 months. Two months prior to due date, coordinators will call patients to assist with scheduling ultrasound exam. Once ultrasound and labs are completed, GI physician will interpret the results to create plan of care (continue with pathway or removed).

Results: Since January 2019, the coordinators have contacted more than 600 patients to complete their HCC surveillance orders. Approximately $92 \%$ of patients participating had at least 1 ultrasound and lab work ordered and completed; $15 \%$ of those patients had no evidence of an ultrasound scheduled in the past year, indicating they were not in a regular surveillance program.

Conclusion: KPMAS's approach to surveillance for HCC is consistent with most society guidelines for monitoring adult patients at high risk for developing HCC; however, the program has the added benefit of using coordinators to increase patient adherence to the recommended screening, which is a known gap in care. Early results indicate the program is succeeding in ensuring most patients with the HCC surveillance order complete the imaging/labs, and that patients previously out-of-care (no ultrasound in past year) have been reengaged with the recommended testing.

\section{P15.05 \\ Insights Into Statewide Payment and Delivery System Reform: Lessons From the lowa State Innovation Model Evaluation}

\section{Tessa Heeren ${ }^{1}$}

\section{${ }^{1}$ Public Policy Center, University of lowa, lowa City, IA}

Background: In 2015, the Iowa Department of Human Services received a 4-year, \$43 million State Innovation Models (SIM) grant to improve the quality of care and population health while simultaneously reducing health system costs. During the 4-year tenure of the Iowa SIM, various health system interventions were pursued, most of which were implemented in preceding SIM grantee states and endorsed by the Center for Medicare \& Medicaid Innovation. Specific Iowa SIM efforts included payment reform (value-based purchasing), building a statewide unifying infrastructure (statewide admission, discharge, and transfer [ADT] alerting), and establishing multisector referral networks (accountable communities of health).

Methods: Qualitative (eg, key informant interviews, meeting minutes) and quantitative sources (eg, survey data and reported outcome measures) of evidence will be used to examine process and outcome measurement. Original SIM goals and the status at the conclusion of the Iowa SIM will be contrasted and interpreted for 3 main initiatives - valuebased purchasing, statewide ADT alerting, and accountable communities of health.

Results: Original outcome measures of progress towards Iowa SIM goals were impacted by the context of the state and federal health landscape. For instance, enrollment goals 
for value-based purchasing contracts were complicated by the introduction and turnover of managed care organizations in the state. In addition, changes in SIM and state leadership impacted the vision and continuity of implementation. These factors had a significant impact — causing program drift and a momentum slump, which created a new challenge of engaging new stakeholders and maintaining the investment from original stakeholders.

Conclusion: Thoughtful development and careful documentation of process outcomes should be required to measure progress in large-scale systems-change initiatives and be used to interpret the summative outcomes of grants. By emphasizing the context and foundational benchmarks of meeting overarching goals, setbacks can be differentiated from "failures" and can inform and motivate the sustainment of grant programming beyond funding.

\section{P15.06}

\section{The Northwest Biobank and Tissue Library: A Valuable Resource for Collaborative Research}

Joanna Bulkley, ${ }^{1}$ Jessica Hunter, ${ }^{1}$ Don Freel, ${ }^{1}$ Allison Naleway, ${ }^{1}$ Charisma Jenkins, ${ }^{1}$ Shawn Westaway, ${ }^{1}$ Lisa Portela-Justice, ${ }^{1}$ Yolanda Prado, ${ }^{1}$ Sheila Weinmann ${ }^{1}$

\section{${ }^{1}$ Kaiser Permanente Northwest, Portland, OR}

Background: The Kaiser Permanente Center for Health Research in Portland, Oregon, houses the Northwest Biobank and Tissue Library (NWBTL), unique biorepositories of blood and tissue specimens linked to clinical, pharmacy, and self-report data from Kaiser Permanente Northwest (KPNW) health plan members. These specimens are available for research projects in collaboration with Center for Health Research investigators.

Methods: Specimens were gathered during research studies and by retaining specimens after usual clinical care with members' permission. Blood-derived specimens include whole blood, plasma, DNA extracted from whole blood, and buffy coat that can be used to obtain genomic data. Tissue specimens include individual formalin-fixed paraffinembedded normal and tumor tissue blocks dating to 1971 as well as associated hematoxylin and eosin (H\&E)-stained slides. Tissue slides can be sectioned to meet thickness, slide type, and staining requirements, and DNA can be extracted. Cervicovaginal lavages collected during pap smears in 1989-1990 also are available. Specimens are linked to each patient's electronic health record, which documents the full scope of our members' medical care. Specimens are also linked to pharmacy and administrative data, the KPNW tumor registry, pathology reports, and genomic data. Some specimens also link to survey data.

Results: The NWBTL contains more than 60,000 bloodderived specimens from more than 40,000 individuals, the majority of whom are female $(56 \%)$ and white $(83 \%)$. Approximately $10 \%$ were collected from patients 80 years of age or older and 9\% from patients younger than 40 . Large disease cohorts include diabetes $(>5000)$, cardiovascular disease $(>3000)$, cancer $(>2800$ breast, prostate, and lung), and clinical depression $(>1300)$. Others include hypertension, hemochromatosis, hip fracture, cerebrotendinous xanthoma (a rare disorder), and human papillomavirus. There are more than 3.5 million normal and tumor tissue blocks and H\&E slides.

Conclusion: Linking KPNW's clinical data with DNA and other biological specimens creates a powerful research resource. NWBTL specimens can be used for a wide range of studies, including studies of molecular and genetic factors associated with disease incidence and progression; pharmacogenetic studies; and disease detection, prevention, or treatment studies. Specimens have been used to predict response to tamoxifen in breast cancer treatment, evaluate the pharmacogenetics of CYP2C19 in proton pump inhibitor use, and identify genetic risk factors for aggressive prostate cancer.

\section{P15.07}

Demographic Differences Between Patients Tested Through the Hepatitis C Pathway Versus Usual Care (non-Pathway)

Fariha Sarwar, ${ }^{1}$ Halina Williams, ${ }^{1}$ Cabell Jonas, ${ }^{1}$ Eric Watson, ${ }^{1}$ Sundeep Basra, ${ }^{1}$ Michael Horberg ${ }^{1}$

\section{${ }^{1}$ Kaiser Permanente Mid-Atlantic States, Rockville, MD}

Background: An estimated 2.4 million people in the United States are living with the hepatitis $\mathrm{C}$ virus (HCV). For some people, $\mathrm{HCV}$ is a short-term illness, but for $70 \%-85 \%$ of people, it becomes a chronic infection. Most infected persons might not be aware of their infection because they are not clinically ill and have no symptoms. There is no vaccine for $\mathrm{HCV}$. The best way to prevent HCV is by avoiding behaviors that can spread the disease. Coordinated care has the potential to improve access to care/treatment for individuals with $\mathrm{HCV}$ who are newly diagnosed, have fallen through the cracks, and are naïve to care and treatment.

Methods: The Hepatitis C Pathway program was developed by the Mid-Atlantic Permanente Medical Group (KPMAS) to close the gaps in diagnosis and care for patients with $\mathrm{HCV}$ infection. All Permanente physicians may order the $\mathrm{HCV}$ screening pathway. $\mathrm{HCV}$ coordinators will communicate these results and schedule follow-up tests to assess current liver health. The coordinators communicate the F-score results to the patients and schedule a consultation with a gastroenterologist to discuss results and treatment options.

Results: We examined Hepatitis C Pathway and nonPathway patients tested from January 2015 to January 2019. Demographic breakdowns showed differences by sex and minor differences by race/ethnicity among those tested. We also examined the differences, by demographics, of those diagnosed with HCV (data not yet available) and those who 
had HCV tests ordered but not resulted. More patients tested through the Hepatitis C Pathway had the recommended HCV RNA testing.

Conclusion: The KPMAS Hepatitis C Pathway, a model for HCV screening, was shown to be particularly effective in ensuring screening among those born from 1945 to 1965 (the Baby Boomer population), which is a U.S. Preventive Services Task Force recommendation. This analysis confirms that patients tested through the Hepatitis C Pathway had closed gaps in care, notably, more had received HCV RNA testing than patients not tested through the program. There do appear to be demographic differences between patients tested via the HCV Pathway versus non-Pathway patients.

\section{GENETICS, GENOMICS, AND PRECISION MEDICINE}

P7.01

"Go Ahead and Screen" - Advice to Health Systems About Regularly Screening for Lynch Syndrome: Interviews With Newly Diagnosed Colon Cancer Patients

Jennifer L. Schneider, ${ }^{1}$ Alison Firemark, ${ }^{1}$ Jim Davis, ${ }^{1}$ Sara Gille, ${ }^{1}$ Andrea Burnett-Hartman, ${ }^{2}$ Laurie Jenson, ${ }^{3}$ Jan Lowery, ${ }^{3}$ Jessica Ezzell Hunter, ${ }^{1}$ Alanna Kulchak $\mathrm{Rahm}^{4}$

${ }^{1}$ Kaiser Permanente Northwest, Portland, OR; ${ }^{2}$ Kaiser Permanente Colorado, Denver, CO; ${ }^{3}$ Catholic Health Initiatives, Englewood, CO; ${ }^{4}$ Geisinger Health, Danville, $P A$

Background: Lynch syndrome (LS) is an inherited cancer syndrome with increased risk for colorectal and endometrial cancers. First-degree relatives of patients with LS have a 50\% chance of also having LS. Universal tumor screening (UTS) for LS among all newly diagnosed cases of colorectal and endometrial cancer is recommended; however, not all health systems have implemented UTS programs. We explored perspectives of patients with colorectal cancer in different health systems on the perceived importance of UTS for LS and their recommendations to health systems.

Methods: To date, we have completed 39 semi-structured telephone interviews among individuals newly diagnosed with colorectal cancer at 4 diverse health care systems. Interviews lasted approximately 60 minutes and were audiorecorded and transcribed for coding and content analysis.

Results: We interviewed 20 males and 19 females, with an average age of 60 years. Most $(80 \%)$ were Caucasian $(n=31)$, and $62 \%(\mathrm{n}=24)$ indicated at least one blood relative receives care within the same health system. The majority (97\%) of patients $(n=38)$ felt it was very important for their health system to regularly screen for LS, as "knowledge is power."
Additional reasons are that the information can guide health care decisions $(\mathrm{n}=14)$ and provide vital information for at-risk relatives $(\mathrm{n}=5)$. However, patients identified 3 concerns: cost of screening to the patient $(\mathrm{n}=4)$; not wanting to be screened without a choice $(n=3)$; and possible feelings of anxiety about learning the LS diagnosis $(n=3)$. About one-third $(n=11)$ felt LS screening should be standard of care at their health system; whereas $15 \%(n=6)$ would prefer some level of patient choice. Most $(90 \%)$ patients $(n=36)$ want their health system to provide proactive education and support about LS screening, including patient-friendly written material $(\mathrm{n}=27)$, links to trusted educational videos and web-based resources $(n=13)$, and assistance from health system staff to further understand LS or communicate with family ( $\mathrm{n}=11)$.

Conclusion: Our initial findings provide novel insight into patient perspectives about the importance for health systems to regularly screen for LS. Patients' advice may guide future implementation of UTS programs to maximize the screening, diagnosis, and education of individuals with LS and their first-degree relatives.

P7.02

Identifying Barriers and Facilitators to Participation in the Kaiser Permanente Research Bank Among Black Members

Musu Sesay, ${ }^{1}$ Brandi Robinson, ${ }^{1}$ Doraina WalkerWilliams, ${ }^{1}$ Drew Bradlyn ${ }^{1}$

\section{${ }^{1}$ Kaiser Permanente Georgia, Atlanta, GA}

Background: Disease burden is prevalent among blacks; however, research participation and, specifically, genetic research requiring a biological sample donation can be challenging, despite the potential benefits. The purpose of this study was to understand black Kaiser Permanente (KP) Georgia members' thoughts on research participation that includes providing a blood sample for genetic analysis, completing a health survey, and granting researchers access to past and future medical record data.

Methods: 35 members were recruited in-person and by phone to participate in focus groups at two KP Georgia locations. Two 90-minute focus groups were conducted in each location. The moderator asked questions, using a semi-structured interview guide, about research knowledge and presentation, historical context, trust, and cultural preferences. Perceptions regarding participation in the KP Research Bank also were discussed.

Results: 13 members participated in 1 of the 4 focus groups. The study population was primarily black $(85 \%)$, nonHispanic (62\%), female (92\%), and 45 years of age or older (62\%). Participants expressed wanting to "give voice," have "black representation" and make a "contribution to their community" as motivating factors to participate in the focus group. Participants noted trustworthiness of researcher and institution, ineffective presentation of research (including 
lack of "black-to-black" communication), associated risks and benefits of a study, and data breach concerns as major barriers to participation. Participants noted the need for education on research subject matters, desire for effective treatments, trust in KP, diversity, and return of research results (personal or group) as facilitators to participating in research. Most participants expressed interest in participating in genetic research if conducted by KP (given their trust and confidence in KP), data are de-identified, information is used only for its intended purposes, and the requested biological samples are collected the same day as recruitment or scheduled conveniently with their next clinic visit. They prefer in-person recruitment and review of consent documents for participation.

Conclusion: Black KP Georgia members are interested in participating in research to make a difference, despite their reservations about historic matters of distrust. Members hope researchers can provide ongoing communications to keep them aware of the difference their contributions in research are making.

\section{P7.03}

\section{Implementing an In-Clinic Recruitment Strategy to Increase Diversity in the Kaiser Permanente Research Bank}

\section{Brandi Robinson,, Doraina Walker-Williams, ${ }^{1}$ Musu Sesay ${ }^{1}$ Marni Segall ${ }^{1}$ Drew Bradlyn ${ }^{1}$}

\section{${ }^{1}$ Kaiser Permanente Georgia, Atlanta, GA}

Background: The Kaiser Permanente (KP) Research Bank is committed to establishing a national biobank that represents KP's richly diverse membership and the communities we serve. National recruiting efforts are primarily web- and email-based and may unintentionally limit diverse participation due to reliance on those methods. KP Georgia implemented an in-clinic recruitment strategy to increase the number of racial and ethnic minorities who consent to participate in the KP Research Bank.

Methods: We implemented an in-clinic recruitment strategy in $9 \mathrm{KP}$ Georgia medical centers with a high proportion of members from racial and ethnic minority groups. Research staff recruited members 1-2 days per week, at tables located near the main entrance of each center. Modest giveaways (eg, pens, lip balm, hand sanitizer) and recruitment flyers were placed on tables to promote the study. Members who approached the recruitment table were asked if they wanted to learn more about the study. If the member expressed interest in learning more, recruiters provided background information about the KP Research Bank and answered any questions. Members who agreed to participate consented electronically using an iPad.

Results: Over the course of 167 recruitment days, 5269 members approached one of our recruitment tables. Of those, $37 \%(n=1938)$ consented to participate in the KP Research
Bank. In comparison, approximately $1 \%$ of KP Georgia members recruited via email consented to participate in the KP Research Bank. Approximately 62\% $(n=1194)$ of members who consented in-clinic were racial or ethnic minorities. Common member questions included: "How is my information protected?" "What will my blood be used for?" and "Have you participated?" When members expressed concerns about participation due to historical cases of research abuses, recruiters listened to the members' concerns, provided information about the measures to protect participants, and discussed the importance of diverse representation in research. Conclusion: Although costly and more intensive than email recruitment, initial results indicate that in-clinic recruitment is an effective strategy to consent racial and ethnic minorities to participate in the KP Research Bank. Engaging in face-toface conversations about the past and future of research was essential to our success in recruiting members from minority populations.

\section{P7.04 \\ Member Attitudes Toward Research and Biobanking Participation}

\author{
Musu Sesay, ${ }^{1}$ Brandi Robinson, ${ }^{1}$ Doraina Walker- \\ Williams, ${ }^{1}$ Drew Bradlyn ${ }^{1}$
}

${ }^{1}$ Kaiser Permanente Georgia, Atlanta, GA

Background: Participation in biobanking research may be impacted by a variety of factors, including race/ethnicity, age, distrust of researchers, privacy concerns, and the potential for personal or community benefit. This study aimed to determine the importance of specific factors in an individual's decision to participate in biobanking research.

Methods: A convenience sample of approximately 10,000 adult ( $\geq 18$ years of age) Kaiser Permanente (KP) Georgia members were invited to complete a survey of attitudes regarding participation in biobanking research. The survey was organized into categories reflecting cultural preference, knowledge and understanding, mode of presentation, trust, and perceptions of research. Item content was developed based on existing literature and themes identified in formative focus groups with KP Georgia members.

Results: 400 members completed the survey (varying item response rates of at least $75 \%$ ). Participants were primarily white $(55 \%)$, non-Hispanic $(85 \%)$, female $(66 \%)$, and 45 years of age or older; nonwhite participants were primarily black $(88 \%)$. The majority had been a KP member for 5 or more years $(56 \%)$, held at least an associate's degree $(60 \%)$, and had previously participated in research $(40 \%)$. Compared to whites, significantly more nonwhites considered it important to be informed about a research study in person $(29 \%$ vs $44 \%$; $\mathrm{P}<0.05)$ by a known/trusted person $(52 \%$ vs $66 \%$; $\mathrm{P}<0.05)$ and to help others ( $75 \%$ vs $86 \%$; $\mathrm{P}<0.05)$, but were less likely to participate if employers/health insurers could use their information against them $(96 \%$ vs $88 \%$; 
$\mathrm{P}<0.05)$. Compared to whites, nonwhites agreed, though not significantly, that researchers could use their information to answer important health questions (99\% vs 100\%; $\mathrm{P}>0.05$ ). They were also likely to participate in research asking for blood samples $(87 \%$ vs $82 \%$; $>0.05)$ or completing health surveys that include sensitive information about drug use and sex $(93 \%$ vs $91 \%$; P>0.05). However, they were less likely to participate in research with the potential for unauthorized access to personal data $(73 \%$ vs $68 \%, \mathrm{P}>0.05)$.

Conclusion: Participants are interested in participating in research to help answer important health questions, but nonwhite participants might not if it violates their privacy or can be used against them for employment or health insurance purposes.

\section{P7.05}

Development of an Access Portal to Coordinate Biobanking Research Applications for the Kaiser Permanente Research Bank

\author{
Suzanne Gillespie, ${ }^{1}$ Alan Bauck, ${ }^{1}$ Terry Chinn, ${ }^{2}$ Renee \\ Trinidad, ${ }^{3}$ Inga Wager ${ }^{2}$
}

${ }^{1}$ Kaiser Permanente Northwest, Portland, OR; ${ }^{2}$ Kaiser Permanente Northern California, Oakland, CA; ${ }^{3}$ Kaiser Permanente Southern California, Pasadena, CA

Background: The Kaiser Permanente Research Bank (KPRB) is one of the most population-diverse biobanks in the United States. Eight geographically distinct Kaiser Permanente regions participate in the KPRB, which contains collections of blood, saliva, and patient data for more than 350,000 individuals. Participants consent to broad use of their biospecimens, electronic health record data, survey data, and genomic data for health research. KPRB reviews all research submissions by performing scientific, communityimpact, and organizational-risk assessments. In addition, KPRB staff review proposals for data and biospecimen feasibility to ensure that the research can be conducted with the resources available and within the projected timeframe. To ensure consistency, monitor researcher requests, and track the process, the KPRB implemented an online workflow tool. Methods: The KPRB Data Coordination Core worked with stakeholders to document the requirements for an online workflow tool and identified 21 predefined criteria that broadly fell into the categories of value, risk, and cost. Those criteria led to the selection of a Kaiser Permanente Information Technology (KPIT) team that specializes in business process management (BPM). The KPIT BPM team leverages software to automate, monitor, and optimize business processes to increase efficiency and reduce costs. The KPIT BPM team worked with stakeholders to document the workflow using IBM's Blueworks Live $^{\circ}$, then used an agile approach to achieve consensus, improve the process model through prototypes, and validate what had been developed. This allowed the stakeholders to refine their ideas and see progress in the build of the application. The result was a tool called Access Portal.

Results: The KPRB Access Portal became available to Kaiser Permanente researchers in January 2018 and to external researchers in August 2018. It is being used to coordinate scientific review, community review, data feasibility review, and biospecimen feasibility review of research proposals.

Conclusion: We are now using the Access Portal to coordinate research proposal reviews and provide feedback to researchers. In addition, use of the Access Portal allows the KPRB to gain further understanding of the submission process and make improvements as needed, as well as to identify gaps in policy, procedures, and technical readiness.

\section{METHODS, DESIGNS, AND ANALYTICS TOOLS}

\section{P13.01}

\section{All-Cause Mortality Production Table From the National Death Index}

\author{
Sundeep Basra, ${ }^{1}$ Monica Ter-Minassian, ${ }^{1}$ Alphonse \\ Derus, ${ }^{1}$ Eric Watson, ${ }^{1}$ Michael Horberg ${ }^{1}$
}

\section{${ }^{1}$ Kaiser Permanente Mid-Atlantic States, Rockville, MD}

Background: The Kaiser Permanente Mid-Atlantic States (KPMAS) virtual data warehouse (VDW) derives its death data from databases sourced from the Social Security Administration, the KPMAS tumor registry, and Clarity. However, more complete information on vital status and cause of death was needed. KPMAS obtained data from the National Death Index (NDI) on select members. We present the matching algorithm and quality control steps used to produce a researcher-ready production member death table.

Methods: All members submitted to NDI were enrolled between 2005 to 2017 and had a date of last contact (identified by an outpatient vital encounter) prior to 2017 . We submitted 12 NDI-required identifying fields, on 1,036,449 members, except for race, state of birth, and marital status. We used the NDI-recommended algorithm to determine the best match, of the multiple matches returned by NDI. We evaluated each field for percentage matched. For a specific matching classification where social security number is missing (class 4), we compared match scores by race and gender. We also compared NDI to the VDW for both vital status and death date for well-matched and poorly matched records.

Results: NDI returned 372,865 (36\%) possible matches, $663,061(64 \%)$ records not matched to the NDI database, and $522(<1 \%)$ rejected records. The total number of resulted death dates from NDI's best score-matching algorithm was 38,861 . The fields of the well-matched records were more than $95 \%$ matched, except for middle initial. Class 4 scores differed significantly by gender and race (Kruskal-Wallis 
$\mathrm{P}<0.0001)$. Member death dates had an exact match between NDI results and the VDW for 27,150 deaths but did not have an exact match for 1628 deaths. There were 10,083 additional deaths from NDI results that were not present in the VDW death data. We found 870 of the poorly matched records had death dates exactly matching VDW.

Conclusion: NDI data can substantially improve the overall capture of deaths. The death dates available in the VDW appeared to be mostly concordant with NDI dates. However, we recommend investigating further quality control measures to ensure the accuracy of NDI's best-match algorithm.

\section{P13.02}

\section{Developing a Toolkit to Calculate Morphine Equivalents and Identify Nonclassified Drugs for Opioid Research}

Phillip Crawford, ${ }^{1}$ Morgan J. Fuoco, ${ }^{1}$ Ladia AlbertsonJunkans, ${ }^{2}$ Arvind Ramaprasan, ${ }^{2}$ Reesa Laws, ${ }^{1}$ Mary Ann McBurnie ${ }^{1}$

${ }^{1}$ Kaiser Permanente Northwest and Hawaii, Portland, OR; ${ }^{2}$ Kaiser Permanente Washington, Seattle, WA

Background: The opioid epidemic is a high priority for many researchers, policymakers, and communities across the United States. Exposure is a primary indicator of risk of addiction, overdose, and death due to opioids. Morphine equivalents (MEQ) are used to standardize variations across drugs to assess the total amount of opioids to which a person has been exposed; however, currently available tools for calculating MEQs have limitations. A standardized, comprehensive approach for computing MEQs is needed. We built on the previous work of a multicenter study of patients prescribed opioids for chronic pain to develop a toolkit with the capacity to process opioid prescribing information from diverse systems and sources and provide a more uniform approach calculating MEQs.

Methods: The toolkit itself was a combination of data, macro programming, and process. Data included in the toolkit was a study-compiled MEQ dataset that included data from multiple sources. Macro programming was used to compare a user's predefined opioid data against the MEQ dataset. This, in combination with user input at key points, accomplished the 6 main steps: 1) identify perfect MEQ matches based on National Drug Code or active ingredient and form matching; 2) identify mid- and high-quality active ingredient and form matches (acceptance/rejection based on additional user review and research; 3) identify gaps in a user's MEQ data by identifying low-quality or nonmatches that require additional user research and/or parsing of text-based strength data; 4) accept user feedback via importable Excel data files that write the data to the local dataset for use in local analysis; 5) when needed, use the Centers for Disease Control and Prevention formula for calculating MEQ; and 6) conditionally accept the drug and MEQ data to build out the parent MEQ dataset.
Documentation for using the toolkit also was created.

Results: Using these methods, a process was developed to make the determination of MEQs a less time-intensive procedure. Working with text-based unstructured strength data is a step in the implementation that could be targeted for additional automation.

Conclusion: Developing a toolkit of resources and procedures for computing standardized MEQs can reduce analyst burden, variation in MEQ definitions, and misclassification of computed MEQs.

P13.03

Measuring the Cost of Caring for Children With a Chronic Health Condition: Design and Description of the Study Population in the r-Kids Study

Joanna Bulkley, ${ }^{1}$ Alexandra Varga, ${ }^{1}$ John Dickerson, ${ }^{1}$ Phillip Crawford, ${ }^{1}$ Katherine Vaughn, ${ }^{1}$ Lisa Croen, ${ }^{2}$ Eric Fombonne, ${ }^{3}$ Brigit Hatch, ${ }^{4}$ Beth Waitzfelder, ${ }^{5}$ Yihe Daida, ${ }^{5}$ April Lee, ${ }^{4}$ Maria Massolo, ${ }^{2}$ Frances Lynch ${ }^{1}$

${ }^{1}$ Kaiser Permanente Northwest, Portland, OR; ${ }^{2}$ Kaiser Permanente Northern California, Oakland, CA; ${ }^{3}$ Oregon Health \& Science University, Portland, OR; ${ }^{4} \mathrm{OCHIN}$, Inc., Portland, OR; ${ }^{5}$ Kaiser Permanente Hawaii, Honolulu, HI

Background: While all families experience financial and time costs related to caring for their child's health, families of children with autism spectrum disorder (ASD) or other chronic health conditions may face a higher burden. Understanding this economic burden is crucial for designing effective policies to support families.

Methods: Using the electronic health record, we identified children between 3 and 16.5 years with ASD, asthma, or neither (GenPop) from 3 Kaiser Permanente regions (Northwest, Hawaii, Northern California) and several health clinics in the OCHIN network. We oversampled racial and ethnic minorities, and matched asthma and GenPop groups to the age and gender distribution of the ASD group. Guardians completed an online survey 3 times, 4 months apart. The survey was comprised of the Family Economic Impact Inventory (measuring financial, time, and employment costs of caring for a child's health), and established instruments measuring the child's quality of life (Pediatric Quality of Life Inventory [PedsQL] and Strengths and Difficulties Questionnaire [SDQ]) and symptom severity for children with ASD (Social Responsiveness Scale [SRS]) or asthma (PROMIS asthma scale). We also assessed parenting stress and parent physical and mental health. All materials were provided in English and Spanish.

Results: 1461 families enrolled in the study (564 ASD, 468 asthma, 429 GenPop). The study children were predominantly male $(79 \%)$, with a mean age of 9.1 years, and ethnically diverse (43\% non-Hispanic white; 21\% Hispanic; 35\% non-Hispanic Asian, black, Native Hawaiian, or other race/ ethnicity). Children in the asthma group were less likely to be 
white (39\%) compared to ASD and GenPop groups (45\% and $46 \%$, respectively; $\mathrm{P}=0.003$ ). The majority of respondents were female $(86 \%)$, had a college degree $(62 \%)$, and were married/partnered (79\%). ASD group respondents were less likely to be employed (73\%) than those in the asthma or GenPop groups (both $80 \%$; $\mathrm{P}=0.023$ ); $49 \%$ of GenPop families had a monthly household income pf $>\$ 6000$, compared to $40 \%$ for both the ASD and asthma groups.

Conclusion: The r-Kids study recruited a large diverse sample. Planned main analyses will examine differences in financial, time, and employment-related costs among study groups. Additional analyses will focus on the impact of family characteristics, ASD severity, employer policies, and caregiver strain on family costs.

P13.04

Human Subjects Considerations in Human Papillomavirus Vaccine Research in Adolescents

\author{
Gabrielle Gundersen, ${ }^{1}$ Aruna Kamineni ${ }^{1}$
}

\section{${ }^{1}$ Kaiser Permanente Washington, Seattle, WA}

Background: State privacy laws can present unique human subjects considerations when conducting human papillomavirus (HPV) vaccine research in minor adolescents. Washington state privacy laws permit 13-17-year-olds to consent to their own mental health, substance use, and reproductive health care. For care related to sexually transmitted infections (STIs), the minimum age for consent is 14 years. At Kaiser Permanente Washington (KPWA), an integrated health care system, HPV vaccination is considered an STI service and is confidential between 14-17-year-olds and their health care provider. Information about this type of care cannot be disclosed to parents without the adolescent's consent.

Methods: In 2018 we sought to conduct a study to understand barriers to HPV vaccine series completion among KPWA members. Human subjects review and approval for this research was more complex than anticipated due to issues of parental consent and assent for minors 14-17 years of age, since HPV vaccination raises privacy concerns. We had originally planned to interview parents of 9-13-year-olds and individuals 14-19 years of age, but we learned we could not approach 14-17-year-olds for their assent to participate in the study without risking disclosure of their HPV vaccination information to their parents without their consent.

Results: After numerous discussions with the KPWA institutional review office and consultations with KPWA legal and privacy departments, we modified our approach strategy and interview plans. We were ultimately approved to interview parents of 11-17-year-olds and young adults 18-19 years of age.

Conclusion: Conducting HPV vaccine research in minor adolescents necessitates allocating additional resources and allowing sufficient time to understand state privacy laws regarding adolescent health care and to engage key stakeholders, including institutional review offices, health care providers who specialize in care of adolescent populations, and, if applicable, legal and privacy departments. Researchers also should be prepared to modify their study to comply with privacy requirements.

\section{P13.05}

\section{Agile Research: Sprinting Through Development of a National Network Website}

\section{Morgan J. Fuoco, ${ }^{1}$ Reesa Laws, ${ }^{1}$ Matt Hornbrook, ${ }^{1}$ Kim Funkhouser, ${ }^{1}$ Mary Ann McBurnie ${ }^{1}$}

\section{${ }^{1}$ Kaiser Permanente Northwest, Portland, OR}

Background: Agile, developed in 2001 in response to traditional waterfall methodology, is an iterative development methodology that is premised on the following 4 values: individuals and interactions, working software, collaboration, and responding to change. Agile values smaller "sprints" of work, each yielding a small functional product. Originally developed to serve the software world, agile methodologies have recently been introduced into other sectors like health care and research. Adapting the agile processes to fit into research environments is a way to balance the reduction in federal funding, tighter timelines, and budget constraints. As part of a national research network, we implemented agile methodology to develop a website to support study activities, including study data collection and management, training and incentive tracking, committee appointment and task management, document management and distribution, institutional review board submissions and documentation, and network staff directory management.

Methods: Two of the core values of agile are iteration and change. The implementation of agile in a research setting has required both iteration and rapid feedback for processes refinement. Our implementation has included: workflow coordination between technical directors and developers; sprint planning, execution, and review; and continual task distillation and adjustment. Flexible work processes have allowed quicker product changes to meet additional requirements.

Results: Generally, the technical directors spend about 2 weeks gathering requirements, writing user stories, developing workflows, and working with the UX developer to develop wireframes. Then, the product moves into development. Initially, we had 4 weeks of development with a week of quality assurance. However, after our sprint retrospective, we decided to divide the work into even smaller sections and have shorter sprints. We also refined how we gather requirements for user stories, work collaboration, and processes for documentation. In 3 months, we have been able to specify and build an initial version of the website that would likely still be under construction using waterfall methodology The agile environment has allowed all team members to offer 
creative solutions to problems that may not be possible under a waterfall approach.

Conclusion: Agile methodology lends itself to collaboration, flexibility, and creativity. Agile offers a way to incorporate the constant associated with research into streamlined development.

\section{PATIENT, CLINICIAN \& HEALTH SYSTEM ENGAGEMENT}

P14.01

Feasibility of Electronically Collecting PatientReported Data From Lower-Income Parents to Integrate and Coordinate Clinical and Community Health Care

Adam Cook, ${ }^{1}$ Jennifer Savage, ${ }^{2}$ Samantha Kling, ${ }^{2}$ Holly Harris, ${ }^{2}$ Michele Marini, ${ }^{2}$ Jacob Mowery, ${ }^{1}$ Shawnee Lutcher, ${ }^{1}$ Lisa Bailey-Davis ${ }^{1}$

${ }^{1}$ Geisinger Health, Danville, PA; ${ }^{2}$ Penn State University, State College, PA

Background: Electronic integration of complementary health services from clinical and community settings may benefit high-risk populations. Parents of economically disadvantaged newborns participate in up to 7 preventive care visits from pediatric primary care providers (PCPs) and Women, Infant, and Children (WIC) nutritionists in the first 6 months of life. In response to stakeholders' concern about conflicting messages and support for an integrated approach, this study describes the feasibility of engaging parents, clinicians, and community nutritionists through electronic data collection and sharing for coordinated care.

Methods: WIC-eligible parents and infants who received care at Geisinger Health were recruited, provided consent, and randomized to intervention or control. The intervention included 1) collecting parent-reported practices via Early Healthy Lifestyles tool; 2) integrating responses into the electronic health record (EHR) to inform care; 3 ) sharing data between clinic and community settings (care coordination opportunities); and 4) coordinating care (interaction with shared data). Data sources included the EHR and WIC data management system. Data were independently coded by 2 study team members for the intervention parent-child dyads. Means and standard deviations are reported.

Results: Caregiver-infant dyads $(\mathrm{N}=131)$ attended 459 $(3.50 \pm 1.03$ per dyad) PCP and 296 (2.26 \pm 0.97 per dyad) WIC visits. Parents completed the Early Healthy Lifestyles risk assessment prior to $53 \%$ of PCP visits $(1.86 \pm 1.15$ per dyad), and these data were integrated into $100 \%$ of infant EHRs. These data were shared with WIC following $100 \%$ of PCP visits and $50 \%$ of the time a WIC visit followed a $\mathrm{PCP}$ visit. Thus, there were $1.76 \pm 0.81$ per dyad PCP-to-WIC care coordination opportunities. When this happened, WIC coordinated care at $67 \%$ of opportunities $(1.18 \pm 0.85$ per dyad). Data were shared with the PCP following $67 \%$ of WIC visits ( $1.5 \pm 0.95$ per dyad), and $59 \%$ of the time a PCP visit followed a WIC visit. Thus, there were $0.89 \pm 0.78$ per dyad WIC-to-PCP care coordination opportunities. When this occurred, PCPs coordinated care at $44 \%$ of opportunities $(0.39 \pm 0.58$ per dyad).

Conclusion: Electronically integrating care is feasible; however, between-participant variability in real-world visit sequence limited care coordination opportunities. Parent input was available for most visits to inform patientcentered care. Workflow integration enabled PCP data sharing, while partial integration limited WIC data sharing. WIC nutritionists were more likely than PCPs to interact with shared data to coordinate care.

P14.02

Determining Whether Opioid Prescription Patterns and Demographics Impact a Clinician's Willingness to Respond to an Opioid-Related Survey

Zijun Shen, ${ }^{1}$ Farah Refai, ${ }^{1}$ William Isenberg, ${ }^{1}$ Amol Navathe, ${ }^{2}$ Mitesh Patel, ${ }^{2}$ Rebecca Pepe, ${ }^{2}$ Kit Delgado, ${ }^{2}$ Joshua Liao, ${ }^{3}$ Xiaowei (Sherry) Yan ${ }^{1}$

${ }^{1}$ Sutter Health, Walnut Creek, CA; ${ }^{2}$ University of Pennsylvania, Philadelphia, PA; ${ }^{3}$ University of Washington, Seattle, WA

Background: We surveyed 451 clinicians from emergency departments (ED) and urgent care (UC) clinics in February 2019 across Sutter Health, a large health care system in Northern California, on their opioid-prescribing behavior and patterns. Clinicians received an email from their local medical director with an explanation of the survey and a link to the questionnaire on SurveyMonkey. Survey responses were collected over a period of 8 weeks, with weekly reminders to nonrespondents. In this study, we seek to understand whether a clinician's opioid prescribing pattern and/or demographic characteristics impact survey response.

Methods: One year of baseline electronic health record (EHR) data from June 2017 to May 2018 was used to capture clinicians' opioid prescription patterns, represented by opioid prescription rate, and basic demographic characteristics. Multivariate logistic regression was used to estimate the association between opioid prescription pattern, clinician demographics, and response rate.

Results: The overall response rate was $55.7 \%(\mathrm{n}=251)$, with response rates for UC clinicians significantly higher than for ED clinicians (69.9\% vs 45.9\%). Nurse practitioners and physician assistants were more likely to respond compared to physicians $(73.7 \%$ vs $54.0 \%$ ). Among ED clinicians, $51.2 \%$ of females and $43.5 \%$ of males responded, whereas among UC clinicians, $66.3 \%$ of females and $74.7 \%$ of males responded. UC clinicians had higher response rates than ED 
clinicians for all levels of years of medical practice (ED: $28.9 \%$ for $<10$ years, $44.2 \%$ for $10-20$ years, $49.6 \%$ for $>20$ years; UC: $76 \%$ for $<10$ years, $68.4 \%$ for $10-20$ years, $70 \%$ for $>20$ years). Neither prescription patterns nor demographic characteristics were associated with response rate for ED clinicians. UC clinicians who had practiced greater than 20 years were less likely to respond (odds ratio: $0.19,95 \% \mathrm{CI}$ : 0.03-0.97) compared to those who practiced for less than 10 years. Opioid prescription patterns were not associated with response rates for either UC or ED responding clinicians.

Conclusion: UC clinicians had significantly higher response rates compared to ED clinicians. Our study reveals that opioid prescription patterns and demographic characteristics do not have an impact on UC and ED clinician response rates, providing validation that information collected from responding clinicians is likely not biased.

\section{P14.03}

\section{What Motivates People With Migraine to Participate in Research: A Qualitative Analysis of Mindfulness- Based Stress Reduction for Migraine}

\section{Heather Law, ${ }^{1}$ Andrew Avins,${ }^{2}$ Alice Pressman ${ }^{1}$ \\ ${ }^{1}$ Sutter Health, Walnut Creek, CA; ${ }^{2}$ Kaiser Permanente Northern California, Oakland, CA}

Background: Migraine is one of the most common neurological disorders in the United States and is ranked among the top 20 causes of disability worldwide. Given the common side effects of pharmaceutical migraine treatments, a substantial number of headache sufferers use integrative medicine to provide symptom relief. Mindfulnessbased stress reduction (MBSR) is an 8-week classroombased intervention that combines meditation and yoga; increasing evidence has demonstrated its effectiveness for a variety of pain-related and functional disorders. As part of a randomized controlled feasibility trial of MBSR for migraine, we conducted a single focus group to learn about participants' experiences in the study.

Methods: We conducted a 2-arm, parallel-comparison randomized controlled feasibility trial of MBSR versus usual care for 60 migraine patients at a large not-forprofit health network in Northern California. The 7 focus group participants all completed the MBSR intervention and all aspects of the study data collection, including 4 headache diaries and questionnaires over a 1-year period. The focus group was audio-recorded and transcribed verbatim. Qualitative data were analyzed with the constant comparative method in order to develop a grounded theory.

Results: Participants $(\mathrm{n}=7)$ reported the following themes: 1) strong preference for integrative medicine and natural methods; 2) enjoyed being part of a group intervention; 3) would not have tried mindfulness on their own; 4) experienced a decrease in frequency and intensity of migraines; and 5) less anxiety and depression.
Conclusion: The focus group was a qualitative exploration of how people with migraine experience the possible benefits and hindrances of participating in a feasibility trial of MBSR for migraine. It was evident that the participants were grateful for the opportunity to be a part of the study and receive the MBSR intervention. MBSR had not only improved their migraines but improved the participants' overall sense of well-being. These findings along with quantitative outcomes data will be used to inform a future fully powered trial. More research is needed to better understand what motivates people with migraine to participate in research studies as well as what types of individuals are more likely to join clinical trials involving integrative medicine.

\section{P14.04}

Introduction of a Knowledgebase to Support a Learning Health Care System

Nico Pronk, ${ }^{1}$ Andrew Alexander, ${ }^{1}$ Richard Paskach, ${ }^{1}$ Santhosh Anand, ${ }^{1}$ Hannah VanLith, ${ }^{1}$ Lauryn Davin ${ }^{1}$

\section{${ }^{1}$ HealthPartners, Bloomington, $M N$}

Background: The development and exchange of knowledge is critical to the success of a learning health care system. HealthPartners Institute chartered a project to implement a knowledgebase for the HealthPartners system to collect, share, and preserve scholarly materials produced throughout HealthPartners. Although there is currently oversight and tracking of research across HealthPartners, the publication listings and citations didn't give users an easy way to search and find publications, education materials, or quality improvement results. In addition, those listings didn't make results available in a concise manner to those external to HealthPartners. By capturing knowledge in a format that is easily accessible, searchable, and includes reporting functionality, these data are better leveraged by the broader organization and community.

Methods: Implementation requirements included a knowledgebase and user interface to search content to understand information related to general learning, research, and quality improvement at HealthPartners, as well as results of previous quality improvement and research projects. Connecting HealthPartners staff to previous initiatives with links to publications, project summary reports, curriculum, etc also was a requirement. Additionally, interfacing with existing citation software to capture scholarly materials, and analysis of metadata on HealthPartners' use of the knowledgebase (areas of interest and number of searches on a subject), were required.

Results: The knowledgebase has brought into focus the value of this information for the broader organization. The deployment of this powerful software demonstrates the "products" of HealthPartners Institute in ways that haven't been seen before. The knowledgebase allows users to have access to publications, profiles, and centers right at their 
fingertips. Google Analytics are used to measure the usage of publications online and in social media, and custom analytics are used to capture search keywords to better understand users and their interests.

Conclusion: The implementation of a knowledgebase has been challenging; however, the immediate benefits are already being realized by HealthPartners Institute: a comprehensive look into the scholarly products of the institute, which in turn demonstrates the value of having an embedded research institute within the enterprise; availability of knowledge for care delivery division to support our learning health care system; and greater visibility of researchers within the organization and externally.

\section{P14.05}

\section{Effect of Navigation Services on Patient Engagement and Activation}

\section{Rebekah Angove, ${ }^{1}$ Kathleen Gallagher, ${ }^{1}$ Eric Anderson ${ }^{1}$ \\ ${ }^{1}$ Patient Advocate Foundation, Hampton, VA}

Background: Patient engagement takes many forms. It encompasses patients being able to define and share their care goals, gather information to make informed choices, engage in shared decision-making with providers, navigate the health and insurance systems, be proactive in their health and care, and expand their advocacy to advise the wider system or community. Success in any (or all) of these activities requires skilled communication, confidence, self-efficacy, and selfadvocacy skills. All of these can be learned or strengthened through training and practice. There is anecdotal evidence to support that many components of patient navigation facilitate the development of these patient engagement skills that we know to be crucial, yet the impact of patient navigation on these aspects of patient engagement has yet to be robustly measured.
Methods: Patient Advocate Foundation delivers navigation and case management services to patients battling lifethreatening and chronic illness. Surveys were administered to all patients served by Patient Advocate Foundation in 2019, both before and after navigation services were provided, to assess the impact of navigation on patient engagement and activation across a variety of domains. These domains include health education, navigation, communication, self-advocacy, and eHealth literacy.

Results: While data collection is still ongoing through December 2019, 200 paired responses have been collected. Preliminary population-level results indicate navigation services reduce general distress and increase confidence in navigating the health care system. Once data collection is complete, matched pair analysis will be done, along with a subanalysis of presenting issues.

Conclusion: Understanding how navigation services impact patient engagement and activation is key to ensuring that these services are meeting the needs of patients and provide evidence for the continued investment by systems and payers. It also will support the identification of opportunities to naturally embed enhanced engagement activities within patient navigation structure and workflow as well as help engineer navigation services that address emergent issues and provide long-lasting effects.

(C) 2020 Aurora Health Care, Inc. 